LA-14396

Approved for public release;

distribution is unlimited.

Complex-Wide Representation of Material

Packaged in 3013 Containers 
Edited by Mable Amador, IRM-CAS

Los Alamos National Laboratory, an Affirmative Action/ Equal Opportunity Employer, is operated by Los Alamos National Security, LLC, for the National Nuclear Security Administration of the U.S. Department of Energy under contract DE-AC52-06NA25396.

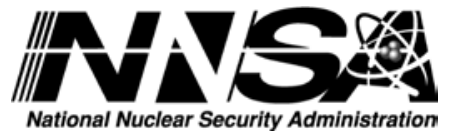

This report was prepared as an account of work sponsored by an agency of the U.S. Government. Neither Los Alamos National Security, LLC, the U.S. Government nor any agency thereof, nor any of their employees make any warranty, express or implied, or assume any legal liability or responsibility for the accuracy, completeness, or usefulness of any information, apparatus, product, or process disclosed, or represent that its use would not infringe privately owned rights. Reference herein to any specific commercial product, process, or service by trade name, trademark, manufacturer, or otherwise does not necessarily constitute or imply its endorsement, recommendation, or favoring by Los Alamos National Security, LLC, the U.S. Government, or any agency thereof. The views and opinions of authors expressed herein do not necessarily state or reflect those of Los Alamos National Security, LLC, the U.S. Government, or any agency thereof. Los Alamos National Laboratory strongly supports academic freedom and a researcher's right to publish; as an institution, however, the Laboratory does not endorse the viewpoint of a publication or guarantee its technical correctness. 
LA-14396

Issued: June 2009

Complex-Wide Representation of Material Packaged in 3013 Containers

Joshua E. Narlesky

Larry G. Peppers

Gary P. Friday*

* Savannah River National Laboratory, Savannah River Site; Aiken, SC 29808. 

The MIS Working Group approves the attached report titled:

Complex-Wide Representation of Material Packaged in 3013 Containers
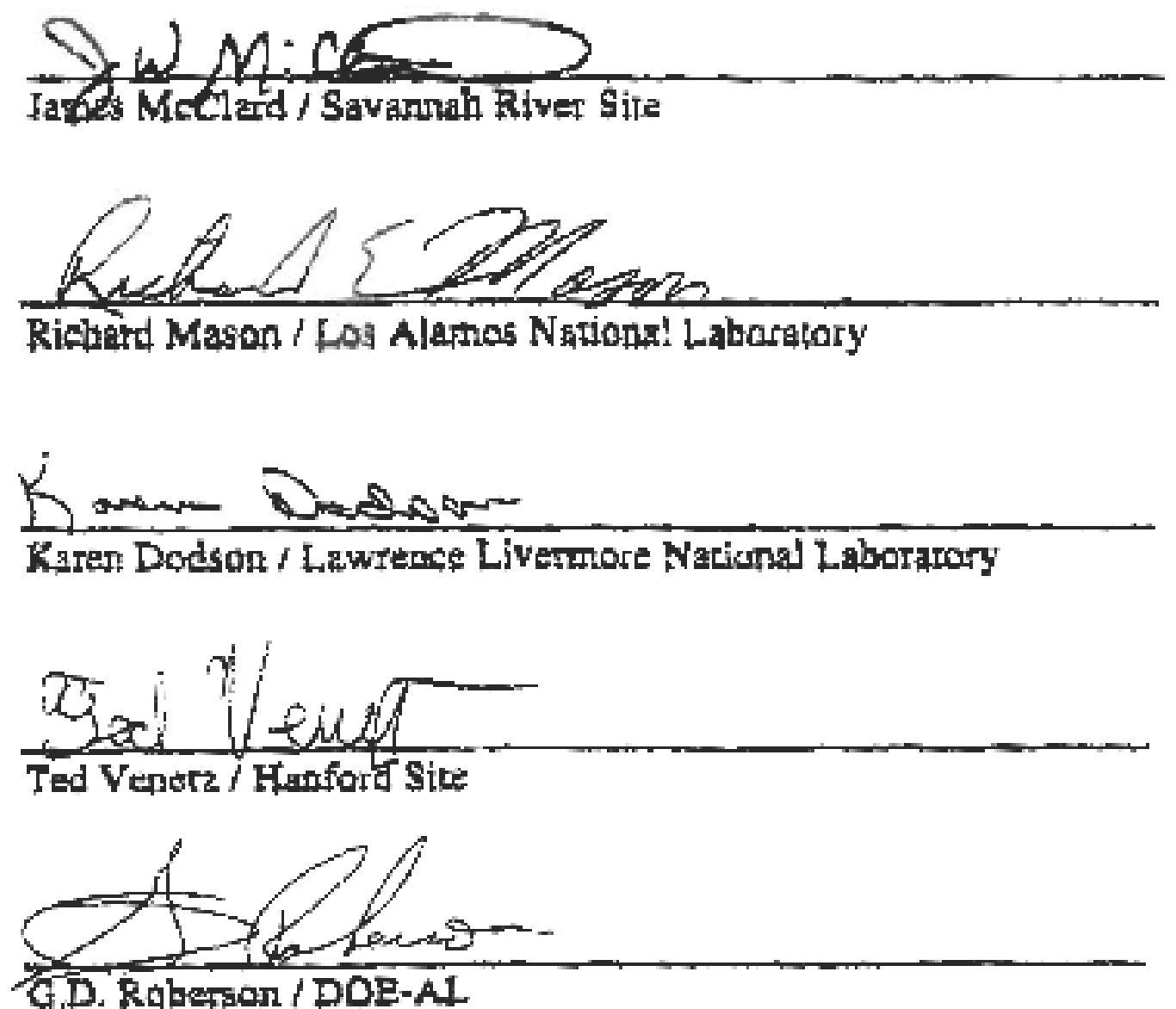



\section{Table of Contents}

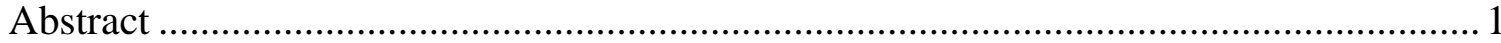

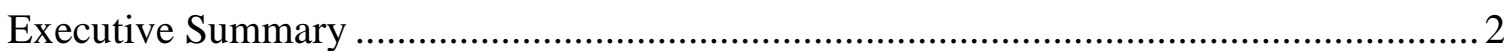

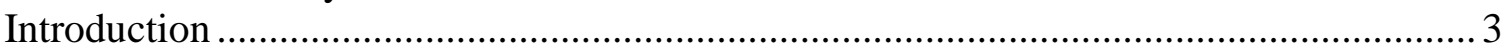

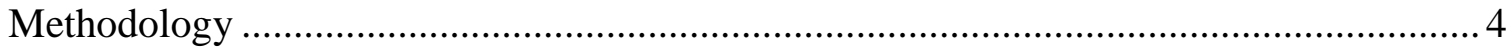

3013 Containers Represented by Process Knowledge .............................................. 5

3013 Containers Represented by MIS Items Showing Unique or Bounding Behavior .. 8

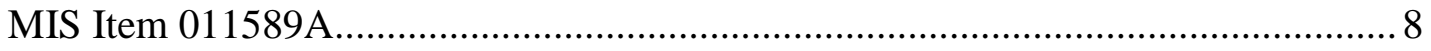

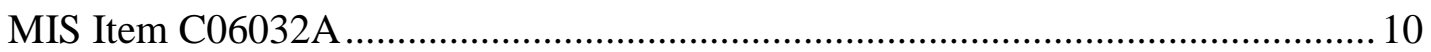

3013 Containers Represented by PG Analysis......................................................... 11

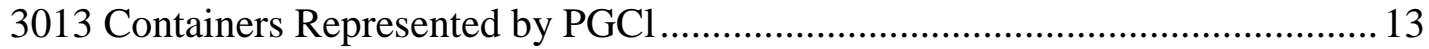

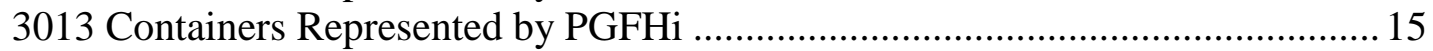

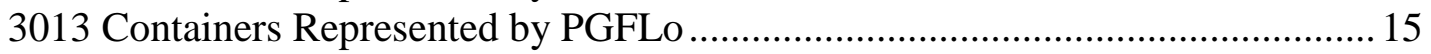

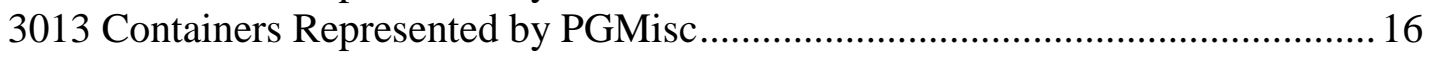

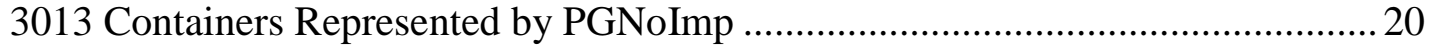

3013 Containers Represented by PG Analysis without a PG Measurement............. 20

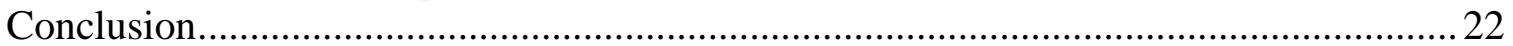

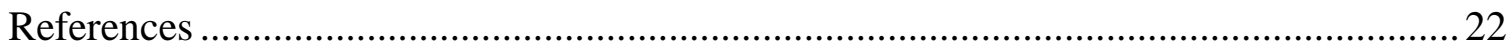

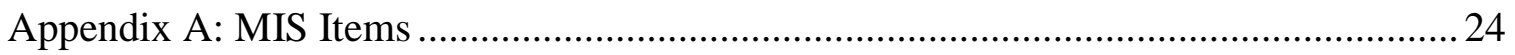

Appendix B: Master "Represented” Table by Process.................................................. 31

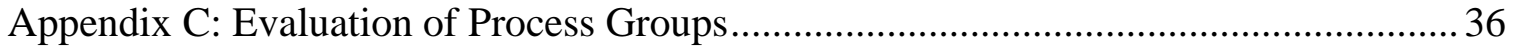

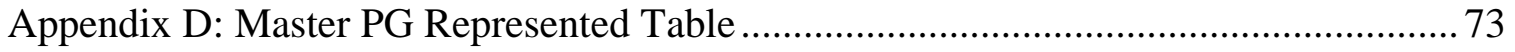

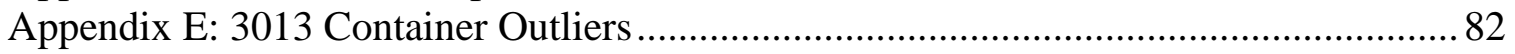





\title{
Complex-Wide Representation of Material Packaged in 3013 Containers
}

by

Joshua E. Narlesky, Larry G. Peppers, and Gary P. Friday

\begin{abstract}
The DOE sites packaging plutonium oxide materials packaged according to Department of Energy 3013 Standard (DOE-STD-3013) are responsible for ensuring that the materials are represented by one or more samples in the Materials Identification and Surveillance (MIS) program. The sites categorized most of the materials into process groups, and the remaining materials were characterized, based on the prompt gamma analysis results. The sites issued documents to identify the relationships between the materials packaged in 3013 containers and representative materials in the MIS program. These "Represented" documents were then reviewed and concurred with by the MIS Working Group. However, these documents were developed uniquely at each site and were issued before completion of sample characterization, small-scale experiments, and prompt gamma analysis, which provided more detailed information about the chemical impurities and the behavior of the material in storage. Therefore, based on the most recent data, relationships between the materials packaged in 3013 containers and representative materials in the MIS program been revised. With the prompt gamma analysis completed for Hanford, Rocky Flats, and Savannah River Site 3013 containers, MIS items have been assigned to the 3013 containers for which representation is based on the prompt gamma analysis results. With the revised relationships and the prompt gamma analysis results, a Master "Represented" table has been compiled to document the linkages between each 3013 container packaged to date and its representative MIS items. This table provides an important link between the Integrated Surveillance Program database, which contains information about each 3013 container to the MIS items database, which contains the characterization, prompt gamma data, and storage behavior data from shelf-life experiments for the representative MIS items.
\end{abstract}




\section{Executive Summary}

Each plutonium-oxide-bearing 3013 container (DOE-STD-3013) is represented by one or more Materials Identification and Surveillance (MIS) items that are believed to reasonably predict the behavior of the material in storage for 50 years. The linkages between the 3013 containers and the MIS items were originally defined by the individual sites with MIS Working Group concurrence and documented in "Represented" documents. However, revisions to the linkages between the 3013 containers and the MIS items were needed to address three issues. First, MIS items 011589A and C06032A exhibited unique or bounding behavior in Small-scale, shelf-life experiments. The behavior of the material in these experiments would not be expected in the entire population of 3013 containers that were originally represented by these items. The MIS working group identified through a detailed analysis of the 3013 container data the 3013 containers that have the highest potential to exhibit this behavior in storage. Second, information became available for both 3013 containers in storage and the MIS items that had not been addressed in the original "Represented" documents issued by the sites. This information showed in certain cases that 3013 containers in some process groups should be represented by a different set of MIS items than what was originally documented. The MIS working group compared the process history information and characterization data of the MIS items to the information available for the material packaged in 3013 containers and identified the MIS items most representative of those 3013 containers. Third, the completion of prompt gamma analysis for the items "represented by prompt gamma” allowed these containers to be directly matched to the representative MIS items.

This report documents the changes to representation and provides a Master "Represented" table for all of the 3013 containers packaged to date. This table provides the link between the Integrated Surveillance Program database that contains information for each 3013 container and the MIS Items database that contains storage behavior information on the related representative materials. This allows the immediate matching of a 3013 container to its representative MIS items and their respective storage behaviors from the Shelf-Life Studies. The methodology presented in this document will be used to determine representation of future produced 3013 containers. 


\section{Introduction}

The Materials Identification and Surveillance (MIS) program $^{1}$ was established within the 94-1 Research and development (R\&D) Program to ensure that plutonium-bearing materials are stabilized, packaged, and safely stored according to Department of Energy 3013 Standard (DOE-STD-3013) ${ }^{2}$ for up to 50 years. As required by the DOE standard, each of the sites was responsible for ensuring that the oxide material packaged in 3013 containers is represented by one or more MIS items. The sites issued "Represented" documents $^{3-7}$ to define representative materials for each process group used by the site, which were then reviewed and concurred with by the MIS Working Group. To predict the storage behavior of individual packaged 3013 containers, a linkage to their representative MIS items is required. The majority of containers in the ISP database use process knowledge to determine representation for the 3013 containers. This method of representation links 3013 containers to MIS items provided by the packaging sites that originated in the same or similar process group. A process group is defined as the process in which the plutonium oxide originated (e.g., oxalate precipitation, pyrochemical, dissolution residuals, etc.). The containers without adequate process knowledge and containers within a given process group that have chemical impurities that are uncharacteristic of that process group will be reviewed by the MIS Working Group and may use prompt gamma analysis to define representation.

All of the information available for individual 3013 containers was collected by the sites in electronic form and sent to Los Alamos National Laboratory (LANL) for inclusion into the complex-wide Integrated Surveillance Program (ISP) database ${ }^{8}$ (the responsibility for maintaining the ISP was subsequently transferred to Savannah River Site in 2006). Similarly, all characterization and small-scale, shelf-life test data were collected by LANL and entered into the MIS Items database ${ }^{9}$ which is maintained by LANL. The "Represented" documents provided the link between the two databases.

The "Represented" documents were issued before completion of sample characterization and small-scale experiments. Additionally, when the represented documents from each site were integrated together, several problems were identified with the linkages between 3013 containers and MIS items. First, two MIS items, 011589A and C06032A, exhibited unique (or bounding) behavior in small-scale, shelf-life studies. ${ }^{10,11} \mathrm{~A}$ comprehensive review of the process history for these items and the process history of the materials stored in 3013 containers showed that these items actually represent fewer 3013 containers than originally presented in the "Represented" documents. Second, revision to linkages between the process groups of 3013 containers and MIS items was needed to incorporate information that was not available when the original "Represented" documents were issued. These data include prompt gamma (PG) analysis, analytical chemistry, Thermogravimetric Analysis (TGA)/Fourier transform infrared spectroscopy (FTIR), and other data for the population of 3013 containers as well as characterization and small-scale, shelf-life data for MIS items. The revisions included the following:

- Linking each process group of 3013 containers to all related items in the MIS inventory, 
- Subdividing process groups that were found to be nonhomogeneous,

- Combining process groups of 3013 containers from process groups having similar impurities and showing similar behavior in small-scale, shelf-life experiments, and

- Ensuring that each process group was represented by MIS items that have both analytical chemistry and small-scale, shelf-life data.

Revisions were also made to the linkages between 3013 containers represented by PG analysis and their associated MIS items. These containers were placed into PG groups (PGGs), based on the impurities identified in the PG spectrum. Each PGG was represented by as many as 11 MIS items of varying actinide content. A query was developed in the ISP database to match each 3013 container to the most similar MIS items.

\section{Methodology}

As part of the 94-1 R\&D program, each DOE site packaging plutonium oxide in 3013 containers selected oxide samples that were believed to be representative of larger populations of material being packaged. Because multiple sites used the same or similar processes, representative MIS materials from one site could be used to represent the same or similar processes from other sites. The MIS items were provided by Rocky Flats Environmental Technology Site (RFETS), Hanford, and LANL and make up the MIS item inventory of representative materials. These samples were shipped to LANL where they became representative MIS items. The MIS items were chemically and physically characterized both before and after stabilization to validate the stabilization process in terms of eliminating the chemical reactivity and reducing the moisture content of the material to below $0.5 \mathrm{wt} \%$. Following characterization, samples of the MIS items were sealed in small-scale test units, and the behavior of the material was monitored over time under 3013 container storage conditions.

All of the current MIS items available for representation are listed in Appendix A grouped according to process. It should be noted that several of the MIS items do not represent any 3013 containers currently in storage. Some MIS items exist in the inventory for research purposes only and were included in the list for completeness. For example, MIS items MISNE2 and MISNE4 were used for moisture and neutron moderation experiments.

Representation of 3013 containers by MIS items is based on one of two criteria. Most containers are represented based on process knowledge, which gives general information as to the impurities that are likely to be present in the material. MIS items in a given group are typical of the 3013 containers in the same process group and provide a starting point for defining representation. For example, all 3013 containers from a burned metal process group would be represented by all MIS items from metal oxidation. Then, adjustments are made to the representation, based on site-specific or process-specific material characteristics. This method of representation was used for most of the 3013 containers. Another method for linking 3013 containers and MIS items into process 
groups is through a detailed analysis of 3013 containers and MIS item data resulting in item-to-item linkages. The item-to-item linkages were reserved for identification of containers represented by MIS items with unique or bounding storage behavior (e.g., 3013 containers similar to MIS items 011589A and C06032A). This method of representation was used for only 111 oxide containers out of the total population. Second, for 3013 containers represented by PG analysis in which process history information was not available or suspected to be inaccurate, representation was defined by comparing the PG spectrum of the 3013 container to the PG spectra for the MIS items and defining the "best” matches. This method of representation was used for 296 containers (see Appendix D). Following a consistency review of the process groups, chemical impurities were identified in 33 containers that are uncharacteristic of the process group to which the containers belong. Representative MIS items have been assigned to these 33 containers based on PG analysis (see Appendix E) that will be used for representation since they are uncharacteristic of the original process group they were assigned. The number of containers represented by each method is given below. A discussion of these methods used to define the 3013 container to MIS item linkages is given in the sections that follow.

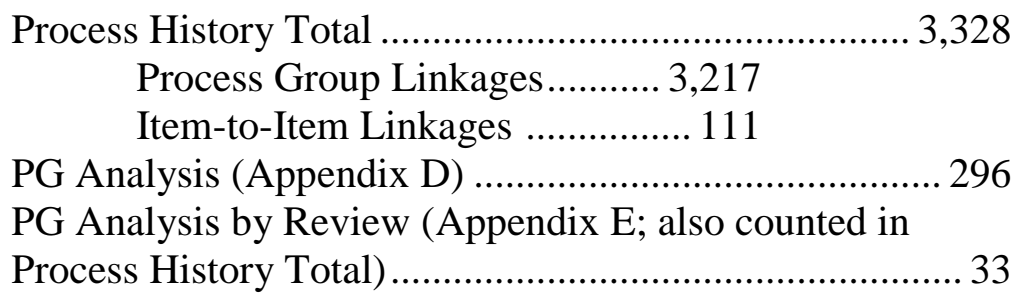

\section{Containers Represented by Process Knowledge}

Several members of the MIS working group and other subject matter experts conducted a review of the linkages between the 3013 container process groups and the MIS items identified in the "Represented" documents provided by the sites. ${ }^{3-7}$ The goal of the review was to ensure that each process group is represented by all related MIS items and to create a combined master representation table. As part of the review, each of the MIS items was first classified into a process group, based on its process history, characterization data, and the results from small-scale, shelf-life experiments. Eleven process groups were identified as part of the classification process and are shown in Table 1. Each process group was then given an identification code called the MIS taxon. 
Table 1. MIS Process Groups

\begin{tabular}{|c|c|c|}
\hline Group ID & Process Group & MIS Taxon* \\
\hline 01 & Oxalate Precipitation & OxIPPt \\
\hline 02 & Peroxide Precipitation & PeroxPPt \\
\hline 03 & Magnesium Hydroxide Precipitation & MgOHPPt \\
\hline 04 & Metal Oxidation & MetalOx \\
\hline 05 & Hydride Oxidation & HydroOx \\
\hline 06 & Pu/U Oxides & PuUOx \\
\hline 07 & Dissolution Residues & DisResd \\
\hline 08 & Oxide Screenings & OxScrns \\
\hline 09 & Pyrochemical Oxide & PyroOx \\
\hline 10 & Scrap Oxide & ScrapOx \\
\hline 11 & Miscellaneous Oxide & MiscOx \\
\hline
\end{tabular}

*The word taxon here is used as a name applied to a taxonomic group in a formal system of nomenclature.

In order to assign individual 3013 containers to a process group, it was noted that many of the process groups contained subgroups. When a process existed at more than one site, material generated by that process at each site was viewed as a distinct subgroup (e.g., oxide from oxalate precipitation generated at Hanford vs. oxide generated at LANL or Savannah River Site (SRS) from oxalate precipitation). In some cases, oxide from the same process group and site was generated from different feed sources or at different times that resulted in variations in the purity of the oxide or introduced different impurities within the process group (e.g., oxide from oxalate precipitation generated at LANL from chloride solution vs. nitrate solution). The 11 groups in Table 1 were expanded to accommodate site-specific subpopulations within some groups and to indicate the packaging site. This combination then becomes the 3013 process group as shown in Table 2.

Each process group in Table 2 was given a unique identification code called the 3013 taxon. This identification code consists of a root and several suffixes. The root corresponds with the MIS process group that represents the material, and the suffixes are used to indicate the site and other site-specific information. 
Table 2. 3013 Container Process Groups

\begin{tabular}{|c|c|c|c|}
\hline Process Group & Packaging Site & Site Specific Information & 3013 Taxon \\
\hline \multirow[t]{7}{*}{ Oxalate Precipitation } & \multirow[t]{4}{*}{ Hanford } & From impure solutions & OxIPPt-HN-Impure \\
\hline & & From pure mixed Pu/U solutions & OxIPPt-HN-MixedPure \\
\hline & & From sources & OxIPPt-HN-Sources \\
\hline & & Product oxide & OxIPPt-HN \\
\hline & \multirow[t]{2}{*}{ Los Alamos } & From chloride line & OxIPPt-LA-Cl \\
\hline & & From nitrate line & OxIPPt-LA-NO3 \\
\hline & Savannah River & Product oxide & OxIPPt-SR \\
\hline Peroxide Precipitation & Rocky Flats & Product oxide & PeroxPPT-RF \\
\hline \multirow{2}{*}{$\begin{array}{l}\text { Magnesium Hydroxide } \\
\text { Precipitation }\end{array}$} & Hanford & & MgOHPPt-HN \\
\hline & Rocky Flats & & MgOHPPt-RF \\
\hline \multirow[t]{6}{*}{ Metal Oxidation } & Hanford & & MetalOx-HN \\
\hline & Los Alamos & & MetalOx-LA \\
\hline & Rocky Flats & & MetalOX-RF \\
\hline & \multirow[t]{3}{*}{ Savannah River } & Produced at Lawrence Livermore & MetalOx-SR-LL \\
\hline & & Produced at Rocky Flats & MetalOx-SR-RF \\
\hline & & & MetalOx-SR \\
\hline Hydride Oxidation & Rocky Flats & & HydroOx-RF \\
\hline \multirow[t]{7}{*}{ Pu/U Oxides } & \multirow[t]{2}{*}{ Hanford } & From alloy oxidation & PuUOx-HN-Alloy \\
\hline & & From oxalate precipitation & PuUOx-HN-Misc \\
\hline & Los Alamos & & PuUOx-LA \\
\hline & Rocky Flats & & PuUOx-RF \\
\hline & \multirow[t]{3}{*}{ Savannah River } & From alloy oxidation & PuUOx-SR-Alloy \\
\hline & & From Hanford & PuUOX-SR-HN \\
\hline & & From oxalate precipitation & PuUOx-SR-Misc \\
\hline \multirow[t]{2}{*}{ Dissolution Residues } & Los Alamos & & DisResd-LA \\
\hline & Rocky Flats & & DisResd-RF \\
\hline Oxide Screenings & Rocky Flats & & OxScrns-RF \\
\hline \multirow[t]{5}{*}{ Pyrochemical Oxide } & \multirow[t]{3}{*}{ Hanford } & From Rocky Flats & PyroOx-HN-RF-ERScrap \\
\hline & & & PyroOx-HN-RF-FndryOX \\
\hline & & & PyroOx-HN-RF-MiscOx \\
\hline & Los Alamos & & PyroOx-LA \\
\hline & Rocky Flats & & PyroOx-RF \\
\hline \multirow[t]{2}{*}{ Scrap Oxide } & \multirow[t]{2}{*}{ Hanford } & & ScrapOx-HN-Hi \\
\hline & & & ScrapOx-HN-Lo \\
\hline \multirow[t]{2}{*}{ Miscellaneous Oxide } & Hanford & From polycubes & MiscOx-HN-PC \\
\hline & Los Alamos & & MiscOx-LA \\
\hline
\end{tabular}

MIS items were then assigned to each of the process groups in Table 2 so that each 3013 process group is represented by all applicable MIS items that match the group. A list of the process groups and their associated MIS items is shown in Appendix B. The 3013 process groups were compared to their respective MIS process groups, based on their assay values and impurities. MIS items having impurities or properties different from those in the process group of 3013 containers were removed from the list. MIS items from other process groups having a similar assay and impurities were added to the list in some cases. For example, 3013 containers with metal oxidation material generated at Hanford was greater than $71 \mathrm{wt} \%$ actinide and did not have chloride or fluoride detected by PG. As shown in Appendix C; Metal Oxidation at Hanford, this material was originally represented by 011589A, 011608, and TS707001. MIS item 011589A was removed because the unique behavior observed in MIS item 011589A is associated with chlorides, and PG did not detect chloride in this process group. MIS items 07221730 and 
ARF-102-85-114-1 were added because they contain high-purity plutonium oxide generated from metal oxidation.

During the review, 3013 containers in some process groups were found by PG analysis to have chemical impurities that are uncharacteristic of the MIS items representing that process group. These containers are considered to be outliers and are listed in Appendix E. PG analysis can be used to better match these containers to MIS items having similar impurities.

Upon completion of the review, the updated linkages between 3013 process groups and MIS items were combined into the master represented table, which is found in Appendix B. The changes to the original "Represented" linkages were documented and are included in Appendix C. The taxons in the master-represented table provide a method for implementing the complex linkages between the ISP database and the MIS items database and allow for generating queries between the two databases. Through the 3013 taxon, each 3013 container can be matched to the MIS process group that represents it, the specific MIS items within the process group that represent the 3013 container, and the characterization and shelf-life data for the MIS items that represent the 3013 container.

\section{Containers Represented by MIS Items Showing Unique or Bounding Behavior}

In the small-scale, shelf-life studies, 10-gram samples of the MIS items are placed in sealed, instrumented test containers with a gas mixture of mainly helium with some air to represent actual containers. In order to examine the boundary conditions allowed by the 3013 Standard, the materials are prepared so that the total moisture content is approximately $0.5 \mathrm{wt} \%$. The sealed containers are then placed in an array heated to $55^{\circ} \mathrm{C}$, and the pressure and gas composition are monitored over time. Two MIS items (011589A and C06032A) exhibited unique or bounding behavior during these experiments, which led to concerns about the materials represented by these items. The data for the affected 3013 containers were reviewed, and it was found that only a subset of those containers have material that could potentially have conditions like those observed in the smallscale, shelf-life studies.

\section{MIS Item 011589A}

MIS item 011589A is an oxide produced before 1990 at Rocky Flats in Building 707 and is associated with plutonium foundry processing. The oxide material was received at LANL as part of the MIS Program in 1997. The chemical and physical properties of the material were characterized upon receipt by LANL. The material was stabilized for two hours at $950^{\circ} \mathrm{C}$. After stabilization, the material was $90 \mathrm{wt} \%$ plutonium oxide and approximately $2 \mathrm{wt} \%$ soluble salt components (magnesium, calcium, sodium, potassium, chlorine, and fluorine) with the remainder made up of insoluble metal oxides. The gas pressure within the test unit loaded with 011589A material at $0.5 \mathrm{wt} \%$ moisture reached a maximum pressure of $225 \mathrm{kPa}$ (32.6 psia) at approximately one year, at which time it contained a mixture of $46 \% \mathrm{H}_{2}$ and $14 \% \mathrm{O}_{2}$ with the remaining being nonflammable 
gases. At 3.4 years, the total corrected pressure had decreased to $203 \mathrm{kPa}$ (29 psia) with $44 \% \mathrm{H}_{2}$ and $11 \% \mathrm{O}_{2} \cdot{ }^{12}$ No other test unit approaches this combination of flammability and pressure.

Based on the original "Represented" documents, 011589A (in conjunction with other MIS items) represented 611 plutonium oxide-bearing 3013 containers from Hanford, Rocky Flats, and SRS as shown below. ${ }^{10}$

Hanford metal oxidation 106

Hanford impure and scrap mixed oxides from alloy oxidation 12

RFETS metal oxidation 364

RFETS Oxides at SRS 72

Metal oxidation from Livermore at SRS .......................... 35

SRS metal oxidation...................................................... 5

SRS impure / scrap mixed oxides from alloy oxidation.... 17

Because of the large number of containers represented by 011589A, a review was done to identify the 3013 containers that are "most similar" to 011589A. Upon conclusion of the review, 17 containers were identified as "most similar" to 011589A, and 31 containers were identified as being "potentially similar" to 011589A. These containers are listed in Tables 3 and 4 and are now the only containers represented by 011589A.

Table 3. Plutonium Oxide-Bearing 3013 Containers Identified as “Most Similar” to MIS Item 011589A

\begin{tabular}{|l|c|c|c|c|}
\hline \multicolumn{5}{|c|}{ Hanford Containers (8) } \\
\hline H002447 & H002809 & H002866 & H003077 & H003343 \\
\hline H003931 & H003989 & H004233 & \\
\hline \multicolumn{5}{|c|}{ RFETS Containers (2) } \\
\hline R600793 & R602498 & \\
\hline \multicolumn{5}{|c|}{ SRS Containers (7) } \\
\hline S001105 & S002117 & S002139 & S002187 & S002221 \\
\hline S002251 & S002277 & & \\
\hline
\end{tabular}


Table 4. Plutonium Oxide-Bearing 3013 Containers Identified as "Potentially Similar to MIS Item 011589A

\begin{tabular}{|c|c|c|c|c|}
\hline \multicolumn{5}{|c|}{ Hanford Containers (11) } \\
\hline H002391 & $\mathrm{H} 002410$ & $\mathrm{H} 002474$ & $\mathrm{H} 002657$ & H002767 \\
\hline H003367 & $\mathrm{H} 003413$ & H003748 & $\mathrm{H} 003774$ & $\mathrm{H} 003940$ \\
\hline H004156 & & & & \\
\hline \multicolumn{5}{|c|}{ RFETS Containers (13) } \\
\hline R600060 & R600142 & R600151 & R600178 & R600234 \\
\hline R600718 & R601558 & R601611 & R601793 & R602012 \\
\hline R602245 & R602787 & R611398 & & \\
\hline \multicolumn{5}{|c|}{ SRS Containers (7) } \\
\hline S002121 & S002125 & S002136 & S002157 & S002203 \\
\hline S002284 & S002286 & & & \\
\hline
\end{tabular}

\section{MIS Item C06032A}

MIS item C06032A also originated at Rocky Flats and was identified as material from oxide screenings. ${ }^{11}$ Oxide screenings was a broad category that included solid chunks of material removed from plutonium oxide during foundry operations and packaging. In foundry operations, solid chunks were separated from the bulk material and calcined in air at $500^{\circ} \mathrm{C}$ to convert any plutonium metal to oxide. Any remaining solids were calcined again. Any remaining solids after the second calcination, which may include tantalum metal, steel, and crucible pieces, were collected and placed in containers designated as oxide screenings (RFETS Item Description Code 159). Oxide screenings generated in packaging operations consisted of solid chunks removed from chloride-bearing plutonium oxides that were packaged for off-site shipment. The specific process history information for C06032A indicates that the oxide screenings were generated from oxides at Rocky Flats packaged for off-site shipment, based on the high chloride content of the material and the building in which it was generated.

The oxide material was received at LANL as part of the MIS Program in 1997, and the chemical and physical properties of the material were characterized upon receipt. During the characterization, the material was split into separate portions so that characterization data could be obtained for the material under different conditions. After stabilization, the milled portion was $74 \mathrm{wt} \%$ plutonium oxide and approximately $24 \%$ soluble salt components (magnesium, calcium, sodium, chloride and fluoride) with the remainder made up of insoluble metal oxides. The gas pressure within the test unit loaded with C06032A material at $0.5 \mathrm{wt} \%$ moisture reached a pressure of $415 \mathrm{kPa}$ (60.1 psia) at approximately one year, at which time it contained a mixture of $65 \% \mathrm{H}_{2}$ with the remaining being nonflammable gases. At 3.8 years, the total corrected pressure had increased to $545 \mathrm{kPa}$ (79.1 psia) with $77 \% \mathrm{H}_{2}$. No other test unit approaches this pressure.

Based on the original "Represented" documents, C06032A (in conjunction with other MIS items) represents oxide screenings from Rocky Flats, impure and scrap oxides from Hanford with 30-80 wt\% plutonium from the Plutonium Finishing Plant (PFP) and impure and scrap mixed oxides from Hanford alloy oxidation. In the subsequent review 
of the ISP database, it was confirmed that C06032A does represent the Rocky Flats oxide screenings material (63 containers). All of these containers are high in chloride as confirmed by PG analysis. Four of these containers have greater than $0.1 \%$ moisture and were identified as being “most similar” to C06032A. However, C06032A does not represent the 185 Hanford containers. Hanford process history states that these groups contain screenings from metal and alloy oxidation, which has considerably lower chloride content as shown by PG analysis. MIS item C06032A is high in chloride, and is therefore not representative of Hanford oxide screenings. The 63 RFETS containers are now the only containers represented by C06032A.

\section{Containers Represented by PG Analysis}

PG analysis offers a powerful and simple semiquantitative analysis of a 3013 container after packaging. In 3013 containers, alpha-particle-induced PG analysis provides an indication of certain major impurities and approximate concentration. Alpha-particleinduced PG analysis is sensitive to aluminum, beryllium, boron, chlorine, fluorine, potassium, phosphorous, magnesium, and sodium. However, there are some impurities, such as calcium, that are not sensitive to PG or have interferences, such as silicon, that make them impossible to detect by PG analysis. The sensitive elements were used to identify groups of containers that have similar prompt gamma signatures and are assumed to have similar behavior in storage. These groups are called prompt gamma groups (PGGs). The PGGs include materials with chloride (PGCl), materials with high concentrations of fluoride without chloride (PGFHi), materials with low concentrations of fluoride without chloride (PGFLo), materials with impurities other than chloride or fluoride (PGMisc), and materials without any impurities detected by PG (PGNoImp). In the ISP database, the PG analysis data can be used to quickly group 3013 containers by impurities present through the assigned PGG. In addition, the PG analysis data are used to determine approximate levels of various chemicals in the containers and to help clarify which process groups to put the 3013 container in.

The 3013 containers without process history information are considered to be "represented by PG analysis," which is defined as the following: "An item is represented by PG analysis if an MIS item exists with the same positive identification for the sensitive elements or if it falls within a specific category (PGG) by impurity content."13 The path between 3013 containers represented by PG analysis and the representative MIS items consists of two segments. One segment was created by assigning MIS items to a PGG, based on its PG signature. The other segment was created by assigning each 3013 container to a PGG, based on its PG signature. The PGGs and associated MIS items are shown in Table 5. All MIS items in this table have been characterized by PG analysis and analytical chemistry and tested in small-scale surveillance experiments. 
Table 5. Linkage of PGGs to Representative MIS Items

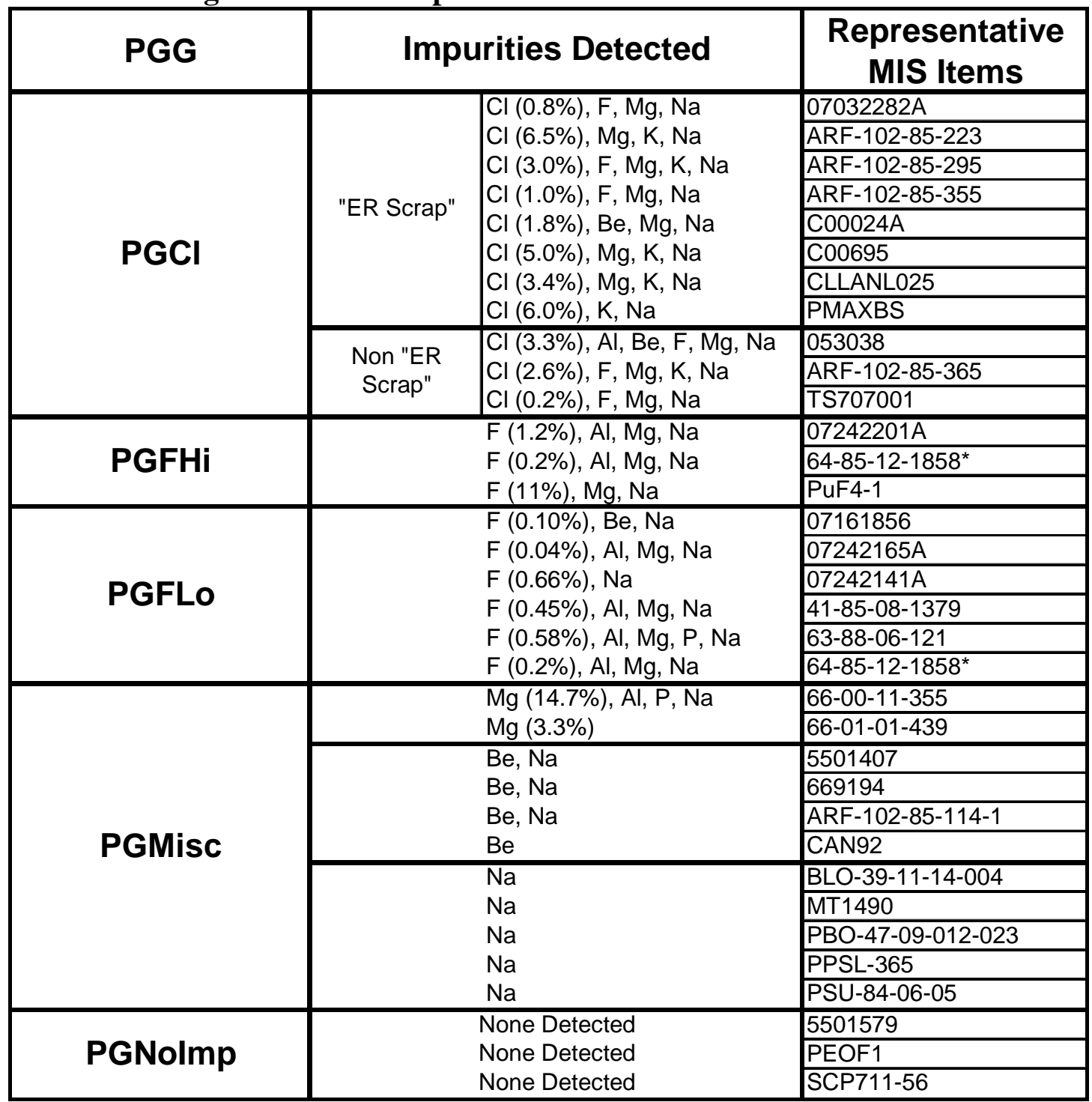

*Note: MIS item 64-85-12-1858 was used to represent both PGFHi and PGFLo. Although the fluorine content best matches PGFLo, this item was added to PGFHi after sorting the 3013 containers because of the similarity of the impurities detected in this item and the impurities detected in the 3013 containers assigned to this group.

Based on the linkages in Table 5, 3013 containers in certain PGGs would be represented by as many as 11 MIS items. The MIS items within a particular PGG may have originated in a variety of processes, have different levels of chemical impurities, and behave differently under storage conditions. Therefore, it is important to link each 3013 container to the most representative MIS items within each PGG. This was accomplished using queries to match each 3013 container to the most similar MIS items, based on the concentration of the chemical impurities as determined by PG analysis and the concentration of the actinides in the material. These queries are discussed in the next sections. The results of the matching are shown in Appendix D. 


\section{Containers Represented by PGCl}

Group PGCl includes 11 MIS items and was matched to 49 packaged 3013 containers, which have a PG analysis indicating the presence of chloride. Estimates for the 3013 container chlorine concentrations range from 0.2 to $8.3 \mathrm{wt} \%$, and the actinide concentrations range from 34.8 to $82.5 \mathrm{wt} \%$. This group can be subdivided, based on the type of chloride that is present in the material. It has been shown that the $\mathrm{Na}: \mathrm{Cl}$ and Mg:Na peak area ratios from PG analysis can be used to distinguish ER scrap from other chloride-bearing plutonium oxides such as foundry oxides. ${ }^{10,14}$ ER scrap materials are expected to have some amount of chloride salt from the salt matrix used in the ER process (equimolar $\mathrm{NaCl} / \mathrm{KCl}$ with $5 \% \mathrm{MgCl}_{2}$ ). The ER scrap materials have $\mathrm{Na}$ :Cl peak area ratios with a mean of 12.0 and a range of 9.5 to 15.5 and $\mathrm{Mg}: \mathrm{Na}$ peak area ratios less than 0.25 . Foundry oxides may be contaminated with chloride salts. The chloride salts originated from skins on the surface of metal buttons that were brushed or burned to oxide and also from occlusions of salt inside buttons. These foundry oxides may also contain varying amounts of Mg and Ca chloride salts from Molten Salt Extraction (MSE) and Direct Oxide Reduction (DOR) processes. As shown by small-scale, shelf-life studies, chloride-bearing foundry oxides such as 011589A behave differently in storage from the ER salts because of the higher concentration of alkaline earth chloride, which absorbs moisture at very low relative humidities. Therefore, $\mathrm{Na}: \mathrm{Cl}$ and $\mathrm{Mg}: \mathrm{Na}$ peak ratios and the chlorine concentration were used as criteria to match each 3013 container to the three most similar MIS items according to the decision tree in Figure 1. Based on the $\mathrm{Na}: \mathrm{Cl}$ and $\mathrm{Mg}: \mathrm{Na}$ peak ratios, ten of the 49 containers appear to have a salt composition similar to that of ER scrap material, and the remaining containers originated in other processes. 


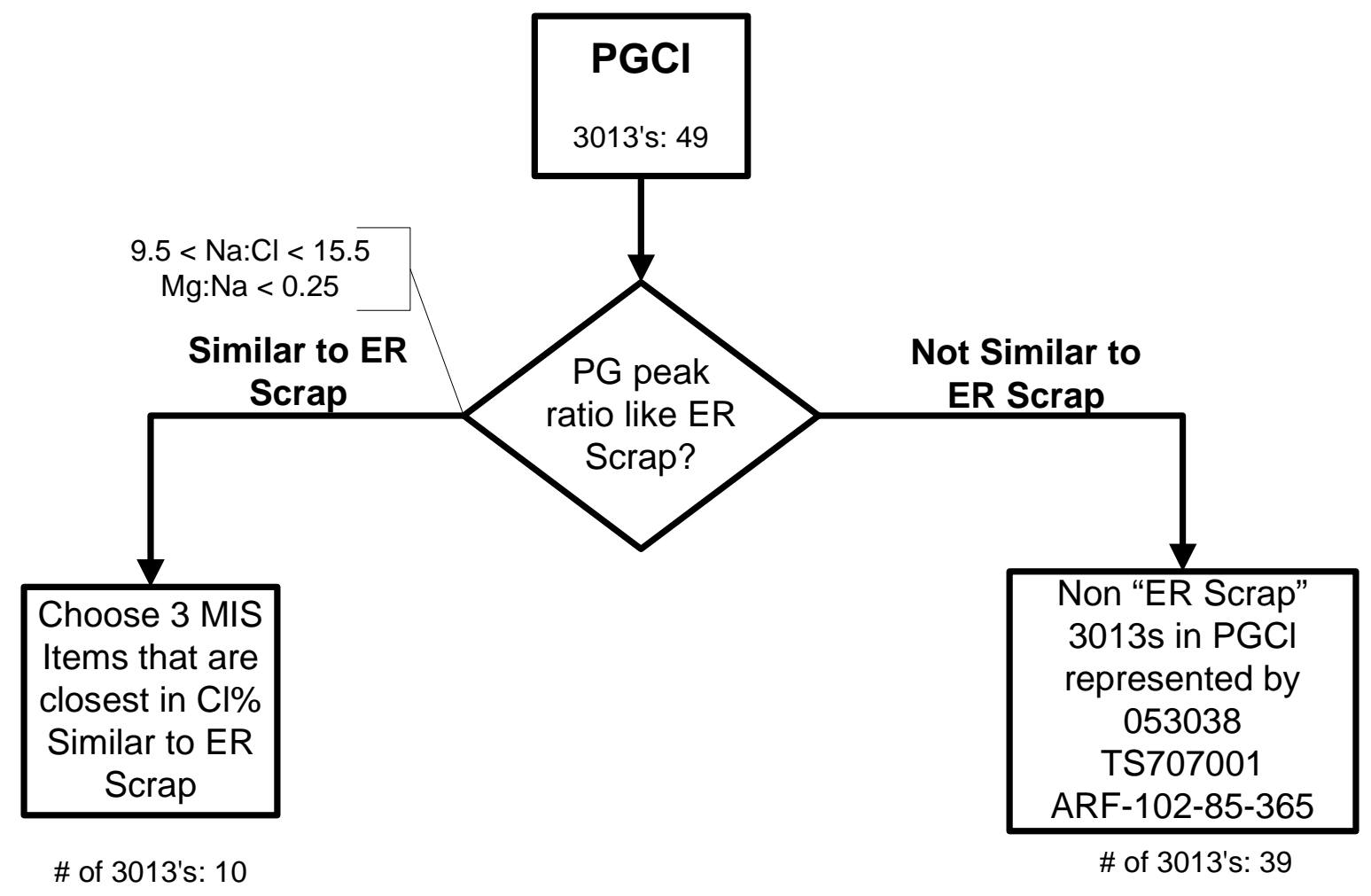

Figure 1. Decision Tree for Matching 3013 Containers in PGCl to the Three Most Similar MIS Items.

Table 6 shows an example of two 3013 containers in PGCl and the respective MIS items that are most similar by this decision tree. The 3013 container H000808 has peak ratios indicating a salt composition similar to that of ER scrap. Based on the decision tree in Figure 1, the three most similar MIS items were chosen according to the chlorine concentration. The peak ratios for H000817 indicate that the salt composition is unlike that of ER scrap; therefore, this container is matched to MIS items 053038, ARF-102-85365, and TS707001, which have chlorine but do not have peak ratios like those of ER scrap. 
Table 6. Example Showing That Two 3013 Containers in PGCI Matched the Most Similar MIS Items Shown Below as Shaded

\begin{tabular}{|c|c|c|c|c|c|c|c|c|c|}
\hline PGG & 3013 ID & Pyro & Act $\%$ & PredCI\% & Item ID & ER & Representative & Act\% (MIS) & Cl\% (MIS) \\
\hline $\mathrm{PGCl}$ & $\mathrm{H} 000808$ & Pyro & 69.72 & 2.78 & CLLANL025 & ER & TRUE & 77.7 & 3.37 \\
\hline $\mathrm{PGCl}$ & $\mathrm{H} 000808$ & Pyro & 69.72 & 2.78 & C00024A & ER & TRUE & 74.3 & 1.79 \\
\hline $\mathrm{PGCl}$ & $\mathrm{H} 000808$ & Pyro & 69.72 & 2.78 & $07032282 \mathrm{~A}$ & ER & FALSE & 67.9 & 0.8 \\
\hline $\mathrm{PGCl}$ & $\mathrm{H} 000808$ & Pyro & 69.72 & 2.78 & ARF-102-85-365 & Non-ER & FALSE & 68.4 & 2.63 \\
\hline $\mathrm{PGCl}$ & $\mathrm{H} 000808$ & Pyro & 69.72 & 2.78 & ARF-102-85-223 & ER & FALSE & 70.9 & 6.5 \\
\hline $\mathrm{PGCl}$ & $\mathrm{H} 000808$ & Pyro & 69.72 & 2.78 & TS707001 & Non-ER & FALSE & 87 & 0.18 \\
\hline $\mathrm{PGCl}$ & $\mathrm{H} 000808$ & Pyro & 69.72 & 2.78 & $\mathrm{C} 00695$ & ER & FALSE & 74.1 & 4.95 \\
\hline $\mathrm{PGCl}$ & $\mathrm{H} 000808$ & Pyro & 69.72 & 2.78 & ARF-102-85-355 & ER & FALSE & 65.6 & 0.96 \\
\hline $\mathrm{PGCl}$ & $\mathrm{H} 000808$ & Pyro & 69.72 & 2.78 & 053038 & Non-ER & FALSE & 64.1 & 3.28 \\
\hline $\mathrm{PGCl}$ & $\mathrm{H} 000808$ & Pyro & 69.72 & 2.78 & PMAXBS & ER & FALSE & 71.9 & 6.02 \\
\hline $\mathrm{PGCl}$ & $\mathrm{H} 000808$ & Pyro & 69.72 & 2.78 & ARF-102-85-295 & ER & TRUE & 39.6 & 2.99 \\
\hline $\mathrm{PGCl}$ & $\mathrm{H} 000817$ & Non-Pyro & 72.22 & 2.38 & CLLANL025 & ER & FALSE & 77.7 & 3.37 \\
\hline $\mathrm{PGCl}$ & $\mathrm{H} 000817$ & Non-Pyro & 72.22 & 2.38 & PMAXBS & ER & FALSE & 71.9 & 6.02 \\
\hline $\mathrm{PGCl}$ & $\mathrm{H} 000817$ & Non-Pyro & 72.22 & 2.38 & $07032282 \mathrm{~A}$ & ER & FALSE & 67.9 & 0.8 \\
\hline $\mathrm{PGCl}$ & $\mathrm{H} 000817$ & Non-Pyro & 72.22 & 2.38 & ARF-102-85-223 & ER & FALSE & 70.9 & 6.5 \\
\hline $\mathrm{PGCl}$ & $\mathrm{H} 000817$ & Non-Pyro & 72.22 & 2.38 & $\mathrm{C} 00695$ & ER & FALSE & 74.1 & 4.95 \\
\hline $\mathrm{PGCl}$ & $\mathrm{H} 000817$ & Non-Pyro & 72.22 & 2.38 & ARF-102-85-295 & ER & FALSE & 39.6 & 2.99 \\
\hline $\mathrm{PGCl}$ & $\mathrm{H} 000817$ & Non-Pyro & 72.22 & 2.38 & $\mathrm{C} 00024 \mathrm{~A}$ & ER & FALSE & 74.3 & 1.79 \\
\hline $\mathrm{PGCl}$ & $\mathrm{H} 000817$ & Non-Pyro & 72.22 & 2.38 & 053038 & Non-ER & TRUE & 64.1 & 3.28 \\
\hline $\mathrm{PGCl}$ & $\mathrm{H} 000817$ & Non-Pyro & 72.22 & 2.38 & ARF-102-85-365 & Non-ER & TRUE & 68.4 & 2.63 \\
\hline $\mathrm{PGCl}$ & $\mathrm{H} 000817$ & Non-Pyro & 72.22 & 2.38 & ARF-102-85-355 & ER & FALSE & 65.6 & 0.96 \\
\hline $\mathrm{PGCl}$ & $\mathrm{H} 000817$ & Non-Pyro & 72.22 & 2.38 & TS707001 & Non-ER & TRUE & 87 & 0.18 \\
\hline
\end{tabular}

\section{Containers Represented by PGFHi}

Group PGFHi is for items with greater than or equal to $0.8 \mathrm{wt} \%$ fluorine. The $0.8 \mathrm{wt} \%$ cutoff value was chosen, based on the detection limit of chloride with PG analysis.

Twenty-two packaged 3013 containers were matched to PGFHi and are represented by two MIS items. The estimated fluorine concentration in the 3013 containers ranges from 0.8 to $4.8 \mathrm{wt} \%$, and the actinide concentration ranges from 35.8 to $81.2 \mathrm{wt} \%$. The two MIS items in PGFHi, PuF4-1 and 07242201A, have actinide concentrations of 72.7 and 63.3 wt\% respectively. To better bound the actinide concentration, MIS item 64-85-121858, which has $32.7 \mathrm{wt} \%$ actinide, was assigned to PGFHi to represent the low purity material (particularly those from Hanford); although its fluorine concentration is 0.2 wt\%, which is below the $0.8 \mathrm{wt} \%$ cutoff value. With this item included in PGFHi, the actinide and fluoride concentrations for the materials packaged in 3013 containers are bounded by those of the MIS items, which are designated as representative of all 3013 containers in this group.

\section{Containers Represented by PGFLo}

Group PGFLo is for items with less than $0.8 \mathrm{wt} \%$ fluorine and includes six MIS items. Forty-five packaged 3013 containers were matched to this group. The estimated fluorine concentration in the 3013 containers ranges from 0.02 to $0.8 \mathrm{wt} \%$, and the actinide concentration ranges from 33.6 to greater than $85 \mathrm{wt} \%$. As done previously for PGCl, each 3013 container was matched to the three most similar MIS items in this group. Each 3013 container was matched first to the MIS item closest in actinide concentration and to two other MIS items that are closest in fluoride concentration. An example is shown in Table 7. Here, container R610475 is first matched to MIS item 07161856, based on actinide content, followed by 41-85-08-1379 and 64-85-12-1858, based on the concentration of fluoride. 
Table 7. Example Showing a 3013 Container in PGFLo Matched the Most Similar MIS Items Shown Below as Shaded

\begin{tabular}{|c|c|c|c|c|c|c|c|c|c|c|}
\hline PGG & $\mathbf{3 0 1 3}$ ID & Act $\%$ & $\begin{array}{c}\text { Pred } \\
\text { F\% }\end{array}$ & $\begin{array}{c}\text { Pred } \\
\text { Be } \%\end{array}$ & Item ID & Representative & $\begin{array}{c}\text { Act\% } \\
\text { (MIS) }\end{array}$ & $\begin{array}{c}\text { F\% } \\
\text { (MIS) }\end{array}$ & $\begin{array}{c}\text { Be\% } \\
\text { (MIS) }\end{array}$ & Level \\
\hline PGFLO & R610475 & 76.67 & 0.33 & 0.56 & $07242165 A$ & FALSE & 34.1 & 0.04 & & \\
\hline PGFLO & R610475 & 76.67 & 0.33 & 0.56 & $63-88-06-121$ & FALSE & 35.7 & 0.58 & & \\
\hline PGFLO & R610475 & 76.67 & 0.33 & 0.56 & $41-85-08-1379$ & TRUE & 34.1 & 0.45 & & PGFLo-2-F\% \\
\hline PGFLO & R610475 & 76.67 & 0.33 & 0.56 & $64-85-12-1858$ & TRUE & 32.7 & 0.21 & & PGFLo-3-F\% \\
\hline PGFLO & R610475 & 76.67 & 0.33 & 0.56 & 07242141 A & FALSE & 43.1 & 0.66 & & \\
\hline PGFLO & R610475 & 76.67 & 0.33 & 0.56 & 07161856 & TRUE & 84.2 & 0.1 & 0.04 & PGFL0-1-Act $\%$ \\
\hline
\end{tabular}

\section{Containers Represented by PGMisc}

Group PGMisc includes materials without chloride or fluoride. Eleven MIS items and 159 miscellaneous 3013 containers were assigned to this group. The actinide concentration of the 3013 containers in this group ranges from 30 to greater than $85 \mathrm{wt} \%$. Uranium is found in many of the containers in concentrations as high as $81 \mathrm{wt} \%$. The MIS items representing this group range from low-purity materials from the magnesium hydroxide precipitation process to high-purity plutonium and mixed plutonium/uranium oxides. None of the materials in this group are significant gas generators in the smallscale, shelf-life studies.

The 3013 containers in this group are expected to be fairly innocuous in their behavior because they are relatively free of chloride salts and other metal impurities. The impurities allowed in this group that PG analysis can detect (aluminum, beryllium, magnesium, phosphorus, sodium) do not have a major effect on the behavior of the material. Although the presence of magnesium and sodium may suggest that chloride salts are present in small amounts, chloride salts at concentrations below the PG detection limit of $0.8 \mathrm{wt} \%$ are generally not of concern for pressurization or corrosion. In some cases, PG analysis provides only limited information because the materials contain impurities that PG analysis cannot detect; however, these impurities are considered to be innocuous.

Because of the limited information from PG analysis for the materials in this group, matching of 3013 containers to the "most similar" MIS items was based primarily on other data in the ISP database. The criteria for matching included the actinide concentration, the uranium concentration (if present), the presence of beryllium (if present), and the ${ }^{240} \mathrm{Pu}$ isotopic concentration. Matching was accomplished using the decision tree in Figure 2.

Examples of the 3013 container to MIS item matching are shown in Table 8. In the first example, ISP data show that 3013 container L000205 is weapons-grade oxide with about $56 \%$ actinide, a small amount of uranium, and no beryllium. The first matching MIS item (CAN92) was chosen, based on the uranium concentration. The second MIS item (66-0101-439) was chosen, based on the actinide concentration, and the third matching MIS item (5501407) was chosen because it is the next closest in actinide concentration. In the second example, H003601 has an actinide concentration of 86.75 wt\%, with no uranium or beryllium, and is high in ${ }^{240} \mathrm{Pu}$. Based on the decision tree in Figure 2, this container is 
represented by ARF-102-85-114-1 and PBO-47-09-012-023, based on its actinide concentration and the absence of uranium and beryllium, and BLO-39-11-14-004, based on its ${ }^{240} \mathrm{Pu}$ content. In the third example, R610497 has an actinide concentration of 83.76 $\mathrm{wt} \%$, and the concentrations of uranium and beryllium in the material are $17.08 \mathrm{wt} \%$ and $0.35 \mathrm{wt} \%$, respectively. Based on the decision tree in Figure 2, this container is represented by 5501407, based on the uranium concentration, CAN92, based on the actinide concentration and the presence of uranium, and ARF-102-85-114-1 because it is a weapons-grade oxide with beryllium. 


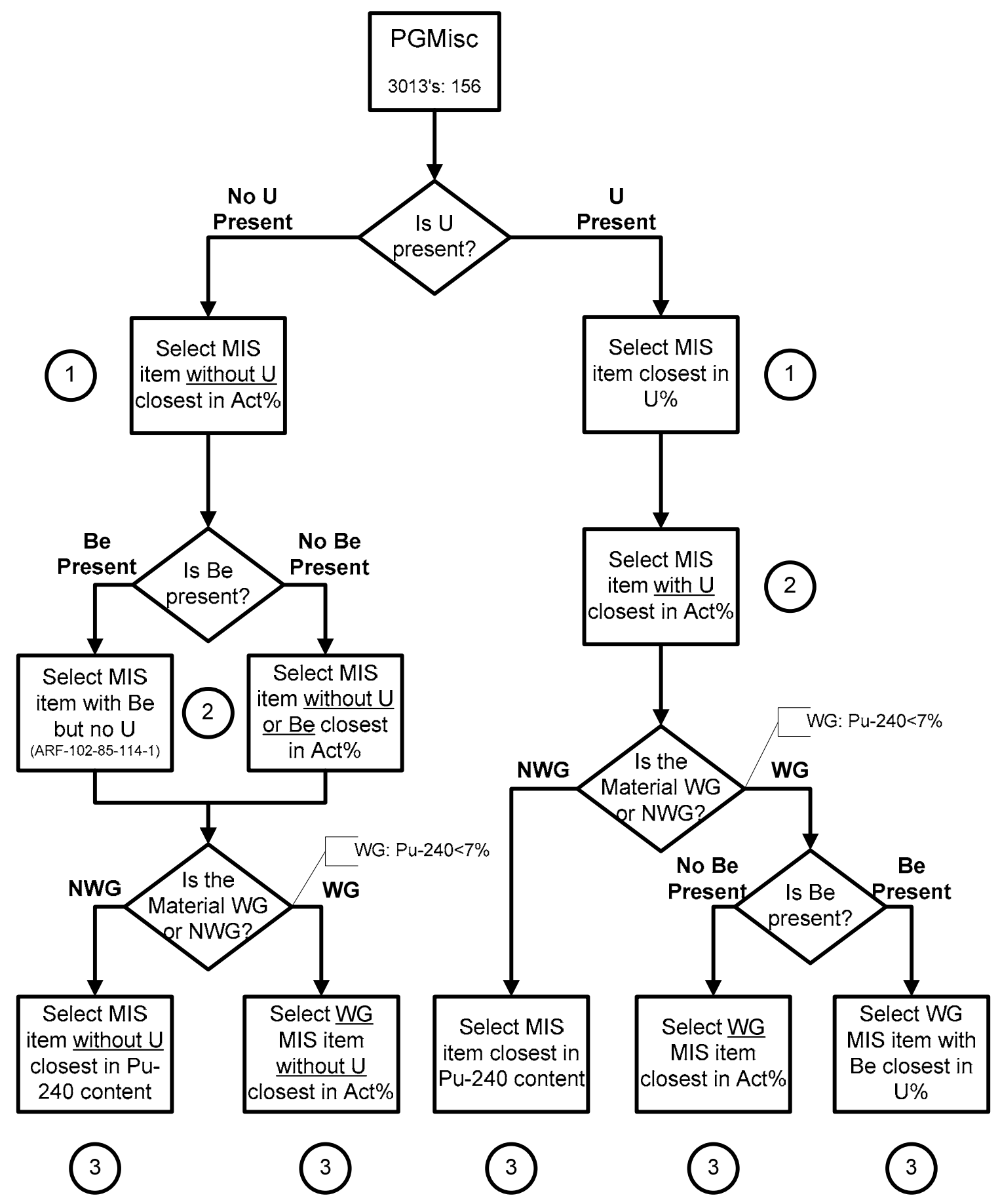

Figure 2. Decision Tree for Matching 3013 Containers in PGMisc to the Three Most Similar MIS Items. 
Table 8. Example Showing Three 3013 Containers in PGMisc Matched the Most Similar MIS Items Shown Below as Shaded

\begin{tabular}{|c|c|c|c|c|c|c|c|c|c|c|c|}
\hline PGG & 3013 ID & Act $\%$ & U\% & PredBe\% & Pu-240 (\%) & Item ID & Representative & Act\% (MIS) & U\% (MIS) & $\mathrm{Be} \%$ (MIS) & Pu-240\% (MIS) \\
\hline PGMisc & L000205 & 56.01 & 0.04 & & 6.9882 & ARF-102-85-114-1 & FALSE & 86.30 & 0.00 & 0.12 & 5.8176 \\
\hline PGMisc & L000205 & 56.01 & 0.04 & & 6.9882 & 5501407 & TRUE & 76.70 & 10.90 & 0.05 & 5.8115 \\
\hline PGMisc & L000205 & 56.01 & 0.04 & & 6.9882 & BLO-39-11-14-004 & FALSE & 85.20 & 0.00 & & 18.8438 \\
\hline PGMisc & L000205 & 56.01 & 0.04 & & 6.9882 & 669194 & FALSE & 83.00 & 69.20 & 0.31 & 4.2964 \\
\hline PGMisc & L000205 & 56.01 & 0.04 & & 6.9882 & PPSL-365 & FALSE & 83.40 & 0.00 & & 6.0355 \\
\hline PGMisc & L000205 & 56.01 & 0.04 & & 6.9882 & $66-01-01-439$ & TRUE & 63.13 & 0.00 & & 5.7306 \\
\hline PGMisc & L000205 & 56.01 & 0.04 & & 6.9882 & MT1490 & FALSE & 85.60 & 0.00 & & 5.9240 \\
\hline PGMisc & L000205 & 56.01 & 0.04 & & 6.9882 & PBO-47-09-012-023 & FALSE & 87.50 & 0.00 & & 12.6022 \\
\hline PGMisc & L000205 & 56.01 & 0.04 & & 6.9882 & PSU-84-06-05 & FALSE & 78.50 & 65.10 & & 8.5417 \\
\hline PGMisc & L000205 & 56.01 & 0.04 & & 6.9882 & $66-00-11-355$ & FALSE & 29.19 & 0.00 & & 5.8041 \\
\hline PGMisc & L000205 & 56.01 & 0.04 & & 6.9882 & CAN92 & TRUE & 83.90 & 2.30 & 0.20 & 5.9480 \\
\hline & & & & & & & & & & & \\
\hline PGMisc & $\mathrm{H} 003601$ & 86.75 & 0.00 & & 21.4068 & 669194 & FALSE & 83.00 & 69.20 & 0.31 & 4.2964 \\
\hline PGMisc & $\mathrm{H} 003601$ & 86.75 & 0.00 & & 21.4068 & $66-00-11-355$ & FALSE & 29.19 & 0.00 & & 5.8041 \\
\hline PGMisc & $\mathrm{H} 003601$ & 86.75 & 0.00 & & 21.4068 & PBO-47-09-012-023 & TRUE & 87.50 & 0.00 & & 12.6022 \\
\hline PGMisc & $\mathrm{H} 003601$ & 86.75 & 0.00 & & 21.4068 & $66-01-01-439$ & FALSE & 63.13 & 0.00 & & 5.7306 \\
\hline PGMisc & $\mathrm{H} 003601$ & 86.75 & 0.00 & & 21.4068 & CAN92 & FALSE & 83.90 & 2.30 & 0.20 & 5.9480 \\
\hline PGMisc & $\mathrm{H} 003601$ & 86.75 & 0.00 & & 21.4068 & PPSL-365 & FALSE & 83.40 & 0.00 & & 6.0355 \\
\hline PGMisc & $\mathrm{H} 003601$ & 86.75 & 0.00 & & 21.4068 & BLO-39-11-14-004 & TRUE & 85.20 & 0.00 & & 18.8438 \\
\hline PGMisc & $\mathrm{H} 003601$ & 86.75 & 0.00 & & 21.4068 & PSU-84-06-05 & FALSE & 78.50 & 65.10 & & 8.5417 \\
\hline PGMisc & $\mathrm{H} 003601$ & 86.75 & 0.00 & & 21.4068 & 5501407 & FALSE & 76.70 & 10.90 & 0.05 & 5.8115 \\
\hline PGMisc & $\mathrm{H} 003601$ & 86.75 & 0.00 & & 21.4068 & MT1490 & FALSE & 85.60 & 0.00 & & 5.9240 \\
\hline PGMisc & $\mathrm{H} 003601$ & 86.75 & 0.00 & & 21.4068 & ARF-102-85-114-1 & TRUE & 86.30 & 0.00 & 0.12 & 5.8176 \\
\hline PGMisc & R610497 & 83.76 & 17.08 & 0.35 & 5.5599 & 5501407 & TRUE & 76.70 & 10.90 & 0.05 & 5.8115 \\
\hline PGMisc & R610497 & 83.76 & 17.08 & 0.35 & 5.5599 & 669194 & FALSE & 83.00 & 69.20 & 0.31 & 4.2964 \\
\hline PGMisc & R610497 & 83.76 & 17.08 & 0.35 & 5.5599 & CAN92 & TRUE & 83.90 & 2.30 & 0.20 & 5.9480 \\
\hline PGMisc & R610497 & 83.76 & 17.08 & 0.35 & 5.5599 & $66-01-01-439$ & FALSE & 63.13 & 0.00 & & 5.7306 \\
\hline PGMisc & R610497 & 83.76 & 17.08 & 0.35 & 5.5599 & PPSL-365 & FALSE & 83.40 & 0.00 & & 6.0355 \\
\hline PGMisc & R610497 & 83.76 & 17.08 & 0.35 & 5.5599 & ARF-102-85-114-1 & TRUE & 86.30 & 0.00 & 0.12 & 5.8176 \\
\hline PGMisc & R610497 & 83.76 & 17.08 & 0.35 & 5.5599 & BLO-39-11-14-004 & FALSE & 85.20 & 0.00 & & 18.8438 \\
\hline PGMisc & R610497 & 83.76 & 17.08 & 0.35 & 5.5599 & MT1490 & FALSE & 85.60 & 0.00 & & 5.9240 \\
\hline PGMisc & R610497 & 83.76 & 17.08 & 0.35 & 5.5599 & PBO-47-09-012-023 & FALSE & 87.50 & 0.00 & & 12.6022 \\
\hline PGMisc & R610497 & 83.76 & 17.08 & 0.35 & 5.5599 & $66-00-11-355$ & FALSE & 29.19 & 0.00 & & 5.8041 \\
\hline PGMisc & R610497 & 83.76 & 17.08 & 0.35 & 5.5599 & PSU-84-06-05 & FALSE & 78.50 & 65.10 & & 8.5417 \\
\hline
\end{tabular}




\section{Containers Represented by PGNolmp}

Group PGNoImp includes materials in which the PG analysis did not detect impurities. This group includes 21 packaged 3013 containers. The actinide concentration for the 3013 containers is $80 \mathrm{wt} \%$ or higher, which shows that these materials are fairly pure. Most of the 3013 containers have uranium present. Three MIS items 5501579, PEOF1, and SCP711-56 represent all 3013 containers in this group. All three of these items are greater than $85 \%$ actinide and have uranium concentrations of $0.1,0.0$, and $70 \mathrm{wt} \%$, respectively.

\section{Containers Represented by PG Analysis without a PG Measurement}

One 3013 container from Rocky Flats (R610887) was represented by PG analysis but did not receive a PG analysis. The ISP data for this container were reviewed, and it was found that this container was from a batch of material that had been split into ten 3013 containers. The relevant data for the other nine 3013 containers is shown in Table 9. The actinide concentration of the material in R610887 is $81.31 \mathrm{wt} \%$, which is within the range of the other nine containers. The PG results for the other nine 3013 containers was similar to those of R610887 in that beryllium was detected in all nine containers, but chlorides and fluorides were not detected in the PG analysis. Therefore, it was assumed that the material in R610887 would not differ significantly from the material in the other nine containers, and the PGMisc decision tree was used to match this container to the most similar MIS items. Based on the PGMisc decision tree, R610887 is represented by MIS items 5501407, ARF-102-85-114-1, and CAN92, all of which originated at Rocky Flats. 
Table 9. Comparison of R610887 with the Nine Related Containers with Material from the Same Input Batch. (Shaded Items Were Selected for Representation.)

\begin{tabular}{|c|c|c|c|c|c|c|c|c|c|c|c|}
\hline PGG & 3013 ID & Act\% & U\% & PredBe\% & Pu-240 (\%) & Item ID & Representative & Act\% (MIS) & U\% (MIS) & $\mathrm{Be} \%$ (MIS) & Pu-240\% (MIS) \\
\hline PGMisc & R610752 & 83.54 & 0.00 & 1.45 & 5.8720 & ARF-102-85-114-1 & TRUE & 86.30 & 0.00 & 0.12 & 5.8176 \\
\hline PGMisc & R610752 & 83.54 & 0.00 & 1.45 & 5.8720 & MT1490 & TRUE & 85.60 & 0.00 & & 5.9240 \\
\hline PGMisc & R610752 & 83.54 & 0.00 & 1.45 & 5.8720 & PPSL-365 & TRUE & 83.40 & 0.00 & & 6.0355 \\
\hline PGMisc & R601843 & 80.18 & 0.00 & 1.31 & 6.3113 & ARF-102-85-114-1 & TRUE & 86.30 & 0.00 & 0.12 & 5.8176 \\
\hline PGMisc & R601843 & 80.18 & 0.00 & 1.31 & 6.3113 & MT1490 & TRUE & 85.60 & 0.00 & & 5.9240 \\
\hline PGMisc & R601843 & 80.18 & 0.00 & 1.31 & 6.3113 & PPSL-365 & TRUE & 83.40 & 0.00 & & 6.0355 \\
\hline PGMisc & R610847 & 87.53 & 0.00 & 1.30 & 5.8720 & ARF-102-85-114-1 & TRUE & 86.30 & 0.00 & 0.12 & 5.8176 \\
\hline PGMisc & R610847 & 87.53 & 0.00 & 1.30 & 5.8720 & MT1490 & TRUE & 85.60 & 0.00 & & 5.9240 \\
\hline PGMisc & R610847 & 87.53 & 0.00 & 1.30 & 5.8720 & PBO-47-09-012-023 & TRUE & 87.50 & 0.00 & & 12.6022 \\
\hline PGMisc & R610874 & 74.78 & 1.07 & 0.89 & 6.2819 & ARF-102-85-114-1 & TRUE & 86.30 & 0.00 & 0.12 & 5.8176 \\
\hline PGMisc & R610874 & 74.78 & 1.07 & 0.89 & 6.2819 & CAN92 & TRUE & 83.90 & 2.30 & 0.20 & 5.9480 \\
\hline PGMisc & R610888 & 91.13 & 0.00 & 1.57 & 5.5798 & ARF-102-85-114-1 & TRUE & 86.30 & 0.00 & 0.12 & 5.8176 \\
\hline PGMisc & R610888 & 91.13 & 0.00 & 1.57 & 5.5798 & MT1490 & TRUE & 85.60 & 0.00 & & 5.9240 \\
\hline PGMisc & R610888 & 91.13 & 0.00 & 1.57 & 5.5798 & PBO-47-09-012-023 & TRUE & 87.50 & 0.00 & & 12.6022 \\
\hline PGMisc & R601928 & 83.76 & 0.00 & 1.35 & 5.8720 & ARF-102-85-114-1 & TRUE & 86.30 & 0.00 & 0.12 & 5.8176 \\
\hline PGMisc & R601928 & 83.76 & 0.00 & 1.35 & 5.8720 & MT1490 & TRUE & 85.60 & 0.00 & & 5.9240 \\
\hline PGMisc & R601928 & 83.76 & 0.00 & 1.35 & 5.8720 & PPSL-365 & TRUE & 83.40 & 0.00 & & 6.0355 \\
\hline PGMisc & R610951 & 82.68 & 1.58 & 1.42 & 6.4735 & 669194 & TRUE & 83.00 & 69.20 & 0.31 & 4.2964 \\
\hline PGMisc & R610951 & 82.68 & 1.58 & 1.42 & 6.4735 & CAN92 & TRUE & 83.90 & 2.30 & 0.20 & 5.9480 \\
\hline PGMisc & R611320 & 74.72 & 0.00 & 1.23 & 6.5979 & ARF-102-85-114-1 & TRUE & 86.30 & 0.00 & 0.12 & 5.8176 \\
\hline PGMisc & R611320 & 74.72 & 0.00 & 1.23 & 6.5979 & MT1490 & TRUE & 85.60 & 0.00 & & 5.9240 \\
\hline PGMisc & R611320 & 74.72 & 0.00 & 1.23 & 6.5979 & PPSL-365 & TRUE & 83.40 & 0.00 & & 6.0355 \\
\hline PGMisc & R611424 & 72.30 & 0.00 & 1.75 & 7.5962 & $66-01-01-439$ & TRUE & 63.13 & 0.00 & & 5.7306 \\
\hline PGMisc & R611424 & 72.30 & 0.00 & 1.75 & 7.5962 & ARF-102-85-114-1 & TRUE & 86.30 & 0.00 & 0.12 & 5.8176 \\
\hline PGMisc & R611424 & 72.30 & 0.00 & 1.75 & 7.5962 & PBO-47-09-012-023 & TRUE & 87.50 & 0.00 & & 12.6022 \\
\hline PGNoPG & R610887 & 81.31 & 0.00 & NM & 5.8720 & 5501407 & TRUE & 76.70 & 10.90 & 0.05 & 5.8115 \\
\hline PGNoPG & R610887 & 81.31 & 0.00 & NM & 5.8720 & $66-00-11-355$ & FALSE & 29.19 & 0.00 & & 5.8041 \\
\hline PGNoPG & R610887 & 81.31 & 0.00 & NM & 5.8720 & 66-01-01-439 & FALSE & 63.13 & 0.00 & & 5.7306 \\
\hline PGNoPG & R610887 & 81.31 & 0.00 & NM & 5.8720 & 669194 & FALSE & 83.00 & 69.20 & 0.31 & 4.2964 \\
\hline PGNoPG & R610887 & 81.31 & 0.00 & NM & 5.8720 & ARF-102-85-114-1 & TRUE & 86.30 & 0.00 & 0.12 & 5.8176 \\
\hline PGNoPG & R610887 & 81.31 & 0.00 & NM & 5.8720 & BLO-39-11-14-004 & FALSE & 85.20 & 0.00 & & 18.8438 \\
\hline PGNoPG & R610887 & 81.31 & 0.00 & NM & 5.8720 & CAN92 & TRUE & 83.90 & 2.30 & 0.20 & 5.9480 \\
\hline PGNoPG & R610887 & 81.31 & 0.00 & NM & 5.8720 & MT1490 & FALSE & 85.60 & 0.00 & & 5.9240 \\
\hline PGNoPG & R610887 & 81.31 & 0.00 & NM & 5.8720 & PBO-47-09-012-023 & FALSE & 87.50 & 0.00 & & 12.6022 \\
\hline PGNoPG & R610887 & 81.31 & 0.00 & NM & 5.8720 & PPSL-365 & FALSE & 83.40 & 0.00 & & 6.0355 \\
\hline PGNoPG & R610887 & 81.31 & 0.00 & NM & 5.8720 & PSU-84-06-05 & FALSE & 78.50 & 65.10 & & 8.5417 \\
\hline
\end{tabular}




\section{Conclusion}

The "Represented" linkages between 3013 containers and MIS items have been revised, based on a review by several members of the MIS working group and other subject matter experts. The revision was needed to address three issues: (1) unique or bounding behavior was observed in MIS items 011589A and C06032A under storage conditions that would not be expected in the entire population of 3013 containers that were originally represented by these items; (2) new information was available for both 3013 containers in storage and the representative MIS items that had not been addressed in the original "Represented" documents issued by the sites; and (3) the completion of PG analysis for the items "represented by PG analysis.” A complete list of MIS Representation linkages has been compiled to create a Master "Represented" table for all of the 3013 containers for all sites. The Master Represented table links the MIS items database to the ISP database and allows the immediate matching of 3013 containers to its representative MIS items and their respective gas-generation data from small-scale, shelflife studies. The linked databases are necessary for field surveillance destructive evaluations, nondestructive evaluations, and 3013 storage activities when the sites need to trace the 3013 containers back to the representative items and data. In addition, the linked databases will enable the sites to simultaneously query data for 3013 containers and MIS items to identify potentially problematic containers. These methodologies will also be applied to demonstrating representativeness for future produced containers as done for the LANL processing groups in Appendix B and C.

\section{References}

1. R.E. Mason, "Integrated Surveillance Program in Support of Long-Term Storage of Plutonium-Bearing Materials,” Los Alamos National Laboratory document LA-UR-00-3246, Rev. 1 (March 2001).

2. Department of Energy, "Stabilization, Packaging, and Storage of PlutoniumBearing Materials,” DOE-STD-3013, U.S. Department of Energy.

3. T.J. Venetz, "PFP Material Representation in the Materials Identification and Surveillance Program,” Fluor Hanford report HNF-14482, Rev. 0 (January 2003).

4. H.F. Dalton, "Rocky Flats Material Representation in the Materials Identification and Surveillance Program,” Department of Energy, Rocky Flats Field Office memorandum (May 7, 2001).

5. R. McNew, "FB-Line Material Representation in the Materials Identification and Surveillance Program,” Westinghouse Savannah River Company report X-TR-F-00003, Revision 0 (October 2003).

6. D.C. Riley, K. Dodson, “Lawrence Livermore National Laboratory (LLNL) Oxide Material Representation in the Material Identification and Surveillance (MIS) Program,” Lawrence Livermore National Laboratory report UCRL-TR202194, Revision 2, (June 29, 2004). 
7. W.A. Punjak, L.G. Peppers, and J.E. Narlesky, "Los Alamos National Laboratory Material Representation in the Materials Identification and Surveillance (MIS) Program,” Los Alamos National Laboratory report LAUR-07-7151 (October 10, 2007).

8. L. Peppers, D. Prochnow, J. Narlesky, and J. Watts, "Integrated Surveillance Program Database,” Los Alamos National Laboratory (Microsoft ${ }^{\circledR}$ Access database available electronically and classified SRD) (2002-2006).

9. J. Narlesky, et. al., "Materials Identification and Surveillance Items Database,” Los Alamos National Laboratory LA-CC-05-111 (2002-Present).

10. G.P. Friday and L.G. Peppers, "Investigation of MIS Item 011589A and 3013 Containers Having Similar Characteristics," Washington Savannah River Company, Savannah River National Laboratory report WSRC-TR-200600236 (August 2006).

11. J.E. Narlesky, J.M. Berg, and D.K. Veirs, "Identification of 3013 Containers Represented by MIS Item C06032A,” Los Alamos National Laboratory document LA-UR-06-6460 (August 2006).

12. J.M. Berg, D.K. Veirs, and L.A. Worl, "Maximum Pressure for Structural Response Calculation of 3013 Containers," Los Alamos National Laboratory report LA-UR-06-4051 (2006).

13. J.E. Narlesky, "PG Analysis of RFETS, Hanford \& LLNL Items" in "FY03 Surveillance \& Monitoring Year End Program Review," Los Alamos National Laboratory report LA-UR-03-9079, October 2003.

14. D.M. Fazzari, S.A. Jones and C.H. Delegard, "Application of Prompt GammaRay Analysis to Identify Electrorefining Salt-Bearing Plutonium Oxide at the Plutonium Finishing Plant,” Pacific Northwest National Laboratory document PNNL-14409 (September 2003). 
Appendix A: MIS Items 
Table A-1. MIS Items Data, (Columns 1 through 7)

\begin{tabular}{|c|c|c|c|c|c|c|}
\hline PROCESS GROUP & SubCategory & $\underset{\text { ID }}{\text { Group }}$ & MIS SAMPLE & $\begin{array}{l}\text { SOURCE } \\
\text { SITE }\end{array}$ & ORIGINATION PROCESS & COMMENTS \\
\hline $\begin{array}{l}\text { Oxalate } \\
\text { Precipitation }\end{array}$ & & 1 & BLO-39-11-14-004 & HANFORD & $\begin{array}{l}\text { Continuous Oxalate } \\
\text { Precipitation/Calcination }\end{array}$ & $\begin{array}{l}\text { Converted from purified nitrate/PFP RMA } \\
\text { Line }\end{array}$ \\
\hline $\begin{array}{l}\text { Oxalate } \\
\text { Precipitation }\end{array}$ & & 1 & CXLNM1 & LANL & LANL Chloride line & $\begin{array}{l}\text { Pure Pu oxide produced from oxalate } \\
\text { precipitated from chloride solution }\end{array}$ \\
\hline $\begin{array}{l}\text { Oxalate } \\
\text { Precipitation }\end{array}$ & & 1 & MISSTD-1 & LANL & Precipitation and Calcination of oxalate & $\begin{array}{l}\text { Batch oxalate (III) precipitation from nitrate } \\
\text { solution and ion-exchange feed. }\end{array}$ \\
\hline $\begin{array}{l}\text { Oxalate } \\
\text { Precipitation }\end{array}$ & & 1 & MISSTD-2 & LANL & Precipitation and Calcination of oxalate & $\begin{array}{l}\text { Batch oxalate (III) precipitation from nitrate } \\
\text { solution and ion-exchange feed }\end{array}$ \\
\hline $\begin{array}{l}\text { Oxalate } \\
\text { Precipitation }\end{array}$ & & 1 & PBO-47-09-012-023 & HANFORD & $\begin{array}{l}\text { Continuous Oxalate } \\
\text { Precipitation/Calcination }\end{array}$ & $\begin{array}{l}\text { Converted from purified nitrate/PUREX N- } \\
\text { cell }\end{array}$ \\
\hline $\begin{array}{l}\text { Oxalate } \\
\text { Precipitation }\end{array}$ & & 1 & PEOF1 & LANL & Precipitation and Calcination of Oxalate & $\begin{array}{l}\text { Batch oxalate (III) precipitation from nitrate } \\
\text { solution and ion-exchange feed. Obtained } \\
\text { from parent PEOFRBJSTD calc at } 975 \text { for } 4 \\
\text { hours. }\end{array}$ \\
\hline $\begin{array}{l}\text { Oxalate } \\
\text { Precipitation }\end{array}$ & & 1 & PEOR3258 & LANL & Precipitation and Calcination of Oxalate & $\begin{array}{l}\text { Batch oxalate (III) precipitation from nitrate } \\
\text { solution and ion-exchange feed. Pure Pu } \\
\text { oxide standard. }\end{array}$ \\
\hline $\begin{array}{l}\text { Oxalate } \\
\text { Precipitation }\end{array}$ & & 1 & CXLOX091802 & LANL & Precipitation and Calcination of Oxlate & $\begin{array}{l}\text { Pure Pu oxide produced from oxalate } \\
\text { precipitated from chloride solution }\end{array}$ \\
\hline $\begin{array}{l}\text { Oxalate } \\
\text { Precipitation }\end{array}$ & & 1 & CXLPROD091901 & LANL & Precipitation and Calcination of Oxlate & $\begin{array}{l}\text { Pure Pu oxide produced from oxalate } \\
\text { precipitated from chloride solution }\end{array}$ \\
\hline $\begin{array}{l}\text { Oxalate } \\
\text { Precipitation }\end{array}$ & & 1 & CXLPROD021202 & LANL & Precipitation and Calcination of Oxlate & $\begin{array}{l}\text { Pure Pu oxide produced from oxalate } \\
\text { precipitated from chloride solution }\end{array}$ \\
\hline $\begin{array}{l}\text { Peroxide } \\
\text { Precipitation }\end{array}$ & & 2 & 07161856 & RFETS & $\begin{array}{l}\text { Precipitation and Calcination of } \\
\text { Peroxide }\end{array}$ & Calcination, Building 771, Room 114 \\
\hline $\begin{array}{l}\text { Peroxide } \\
\text { Precipitation }\end{array}$ & & 2 & 1000089 & RFETS & $\begin{array}{l}\text { Precipitation and Calcination of } \\
\text { Peroxide }\end{array}$ & $\begin{array}{l}\text { Precipitation/Calcination, Building 371, } \\
\text { Room } 3511\end{array}$ \\
\hline $\begin{array}{l}\text { MgOH } \\
\text { Precipitation }\end{array}$ & & 3 & 39-01153A & RFETS & $\begin{array}{l}\text { By-product Oxide from Hydroxide } \\
\text { Precipitation }\end{array}$ & $\begin{array}{l}\text { Caustic Waste Treatment, Building 371, } \\
\text { Room } 1115\end{array}$ \\
\hline $\begin{array}{l}\text { MgOH } \\
\text { Precipitation }\end{array}$ & & 3 & 66-00-11-355 & HANFORD & $\mathrm{Mg}(\mathrm{OH}) 2$ Precipitation/Calcination & From Impure Nitrate (Concentrated Filtrate) \\
\hline $\begin{array}{l}\text { MgOH } \\
\text { Precipitation }\end{array}$ & & 3 & 66-01-01-439 & HANFORD & $\mathrm{Mg}(\mathrm{OH}) 2$ Precipitation/Calcination & From Purified Nitrate \\
\hline Metal Oxidation & & 4 & 011589A & RFETS & Metal Oxidation & $\begin{array}{l}\text { Thermal Stabilization, Building 707, J- } \\
\text { Module Before } 1990\end{array}$ \\
\hline Metal Oxidation & & 4 & 011608 & RFETS & Metal Oxidation & $\begin{array}{l}\text { Thermal Stabilization, Building 707, J- } \\
\text { Module Before } 1990\end{array}$ \\
\hline Metal Oxidation & & 4 & 07221730 & RFETS & Metal Oxidation & $\begin{array}{l}\text { Metal and Chip Burning, Building 771, Room } \\
114\end{array}$ \\
\hline Metal Oxidation & & 4 & 101707001 & RFETS & Metal Oxidation & Foundry Oxide \\
\hline Metal Oxidation & & 4 & ARF-102-85-114-1 & HANFORD & Metal Oxidation & $\begin{array}{l}\text { WG Foundry Oxide received from Rocky } \\
\text { Flats }\end{array}$ \\
\hline Metal Oxidation & & 4 & MT1490 & RFETS & High-Purity Plutonium Oxide Bearing & $\begin{array}{l}\text { Plutonium Metallurgy R\&D, Building } 771, \\
\text { Room } 182\end{array}$ \\
\hline
\end{tabular}


Table A-1. MIS Items Data, (Columns 1 through 7)

\begin{tabular}{|c|c|c|c|c|c|c|}
\hline PROCESS GROUP & SubCategory & $\underset{\text { ID }}{\text { Group }}$ & MIS SAMPLE & $\begin{array}{l}\text { SOURCE } \\
\text { SITE }\end{array}$ & ORIGINATION PROCESS & COMMENTS \\
\hline Metal Oxidation & & 4 & TS707001 & RFETS & Metal Oxidation & $\begin{array}{l}\text { Thermal Stabilization, Building 707, J- } \\
\text { Module After } 1995\end{array}$ \\
\hline Metal Oxidation & & 4 & TS707013 & RFETS & Metal Oxidation & $\begin{array}{l}\text { Thermal Stabilization, Building 707, J- } \\
\text { Module After } 1996\end{array}$ \\
\hline Hydride Oxidation & & 5 & 5501407 & RFETS & $\begin{array}{l}\text { Hydride Oxide / By-product Plutonium- } \\
\text { Uranium Oxide }\end{array}$ & $\begin{array}{l}\text { Hydride Operations Building 779, Rooms } \\
152 \mathrm{~A} / 160 \mathrm{~A}\end{array}$ \\
\hline Hydride Oxidation & & 5 & 5501579 & RFETS & $\begin{array}{l}\text { Hydride Oxide / By-product Plutonium- } \\
\text { Uranium Oxide }\end{array}$ & $\begin{array}{l}\text { Hydride Operations Building 779, Rooms } \\
\text { 152A / 160A }\end{array}$ \\
\hline Hydride Oxidation & & 5 & 669194 & RFETS & By-product Plutonium-Uranium Oxide & Special Assembly Projects, Building 777 \\
\hline Pu/U Oxide & Misc Pu/U Oxides & 6 & 053038 & RFETS & By-product Plutonium-Uranium Oxide & Hydroxide Precipitation, Building 771 \\
\hline Pu/U Oxide & Misc Pu/U Oxides & 6 & CAN92 & RFETS & By-product Plutonium-Uranium Oxide & $\begin{array}{l}\text { Analytical Lab Production Support, Building } \\
559\end{array}$ \\
\hline Pu/U Oxide & Misc Pu/U Oxides & 6 & MISNE2 & LANL & $\begin{array}{l}\text { Mix of } 7221730, \text { TS707001, 11608, and } \\
62750\end{array}$ & $\begin{array}{l}\text { MISNE2 post neutron moderation. Contains } \\
\text { borax and gypsum. }\end{array}$ \\
\hline Pu/U Oxide & Misc Pu/U Oxides & 6 & PuUOXBC05 & LANL & Unknown & $\begin{array}{l}\text { Impure Pu/U oxide originally packaged Nov } \\
\text { 1983; High } \mathrm{Ca}, \mathrm{Mg}, \mathrm{K}, \mathrm{Na}\end{array}$ \\
\hline Pu/U Oxide & MOX Fuel & 6 & MOXSCP-COM & LANL & Calcined MOX & MOX fuel pellets and powder \\
\hline Pu/U Oxide & MOX Fuel & 6 & SCP711-46 & LANL & Hot Plate Oxidation & Fuel Pellets and powder \\
\hline Pu/U Oxide & MOX Fuel & 6 & SCP711-56 & LANL & Hot Plate Oxidation & Fuel Pellets and powder \\
\hline Pu/U Oxide & MOX Fuel & 6 & 1685 & LANL & Misc. & Depleted Uranium Oxide \\
\hline Pu/U Oxide & MOX Fuel & 6 & BMU & LANL & By-product uranium oxide & Burned uranium metal \\
\hline Pu/U Oxide & Pu/U Mixture & 6 & BMUCXL70-30 & LANL & $\begin{array}{l}\text { Mixture of } 70 \% \mathrm{BMU}(\mathrm{EU}) \text { and } 30 \% \\
\mathrm{CXL}(\mathrm{PuO} 2)\end{array}$ & Pu/U mixture \\
\hline Pu/U Oxide & Pu/U Mixture & 6 & BMUCXL93-7 & LANL & $\begin{array}{l}\text { Mixture of } 93 \% \mathrm{BMU}(\mathrm{EU}) \text { and } 7 \% \mathrm{CXL} \\
(\mathrm{PuO})\end{array}$ & Pu/U mixture \\
\hline Pu/U Oxide & Pu/U Mixture & 6 & CXL1685 & LANL & Mixture of $\mathrm{CXL}$ and 1685 & Pu/U mixture \\
\hline Pu/U Oxide & Pu/U Mixture & 6 & CXLBMU70-30 & LANL & $\begin{array}{l}\text { Mixture of } 70 \% \mathrm{CXL}\left(\mathrm{PuO}_{2}\right) \text { and } 30 \% \\
\text { BMU (EU) }\end{array}$ & $\mathrm{Pu} / \mathrm{U}$ mixture \\
\hline Pu/U Oxide & Pu/U Mixture & 6 & CXLBMU93-7 & LANL & $\begin{array}{l}\text { Mixture of } 93 \% \mathrm{CXL}\left(\mathrm{PuO}_{2}\right) \text { and } 7 \% \\
\text { BMU (EU) }\end{array}$ & Pu/U mixture \\
\hline Pu/U Oxide & Polycube Oxide & 6 & PSU-84-06-05 & HANFORD & Recovery from Pyrolytic Processing & Mixed Oxide recovered from polycube/PFP \\
\hline \begin{tabular}{|l|} 
Dissolution \\
Residuals
\end{tabular} & & 7 & 07032282A & RFETS & $\begin{array}{l}\text { Dissolution Residuals (from foundry } \\
\text { scrap oxide) }\end{array}$ & Oxide Dissolution, Building 771, Room 114 \\
\hline \begin{tabular}{|l|} 
Dissolution \\
Residuals
\end{tabular} & & 7 & 07242165A & RFETS & $\begin{array}{l}\text { Dissolution Residuals (from foundry } \\
\text { scrap oxide) }\end{array}$ & $\begin{array}{l}\text { Residue Dissolution, Building 771, Room } \\
\text { 149, Line } 24\end{array}$ \\
\hline $\begin{array}{l}\text { Dissolution } \\
\text { Residuals } \\
\end{array}$ & & 7 & $07242201 \mathrm{~A}$ & RFETS & $\begin{array}{l}\text { Dissolution Residuals (from foundry } \\
\text { scrap oxide) }\end{array}$ & $\begin{array}{l}\text { Residue Dissolution, Building 771, Room } \\
149 \text {, Line } 24 .\end{array}$ \\
\hline $\begin{array}{l}\text { Dissolution } \\
\text { Residuals }\end{array}$ & & 7 & $07242243 \mathrm{~A}$ & RFETS & $\begin{array}{l}\text { Dissolution Residuals (from foundry } \\
\text { scrap oxides) }\end{array}$ & Low Purity Heel \\
\hline \begin{tabular}{|l|} 
Dissolution \\
Residuals
\end{tabular} & & 7 & $07242326 \mathrm{~A}$ & RFETS & $\begin{array}{l}\text { Dissolution Residuals (from foundry } \\
\text { scrap oxides) }\end{array}$ & Dissolution Heel \\
\hline
\end{tabular}


Table A-1. MIS Items Data, (Columns 1 through 7)

\begin{tabular}{|c|c|c|c|c|c|c|}
\hline PROCESS GROUP & SubCategory & $\underset{\text { ID }}{\text { Group }}$ & MIS SAMPLE & $\begin{array}{l}\text { SOURCE } \\
\text { SITE }\end{array}$ & ORIGINATION PROCESS & COMMENTS \\
\hline \begin{tabular}{|l|} 
Dissolution \\
Residuals \\
\end{tabular} & & 7 & 62750 & RFETS & By-product Plutonium-Uranium Oxide & Dissolution, Building 371, Room 1115 \\
\hline $\begin{array}{l}\text { Dissolution } \\
\text { Residuals }\end{array}$ & & 7 & ARF-102-85-355 & HANFORD & Oxide from Residue Processing & WG Oxide received from Rocky Flats \\
\hline Oxide Screenings & & 8 & $07242141 \mathrm{~A}$ & RFETS & $\begin{array}{l}\text { Screenings from Oxide packaged for off } \\
\text { site shipment }\end{array}$ & Unknown origin \\
\hline Oxide Screenings & & 8 & $\mathrm{C} 06032 \mathrm{~A}$ & RFETS & $\begin{array}{l}\text { Screenings from Oxide packaged for off } \\
\text { site shipment }\end{array}$ & $\begin{array}{l}\text { Split Can, Calcined then stored in the B371 } \\
\text { S/R. }\end{array}$ \\
\hline $\begin{array}{l}\begin{array}{l}\text { Pyrochemical } \\
\text { Oxide }\end{array} \\
\end{array}$ & & 9 & 520610020 & RFETS & Oxide from Pyrochemical Processes & $\begin{array}{l}\text { Pyrochemistry Technology Development, } \\
\text { Building } 779\end{array}$ \\
\hline \begin{tabular}{|l}
$\begin{array}{l}\text { Pyrochemical } \\
\text { Oxide }\end{array}$ \\
\end{tabular} & & 9 & ARF-102-85-223 & HANFORD & $\begin{array}{l}\text { Scrap Oxide from Pyrochemical } \\
\text { Process }\end{array}$ & WG Oxide received from Rocky Flats \\
\hline $\begin{array}{l}\text { Pyrochemical } \\
\text { Oxide }\end{array}$ & & 9 & ARF-102-85-295 & HANFORD & $\begin{array}{l}\text { Scrap Oxide from Pyrochemical } \\
\text { Process }\end{array}$ & WG Oxide received from Rocky Flats \\
\hline \begin{tabular}{|l|} 
Pyrochemical \\
Oxide
\end{tabular} & & 9 & ARF-102-85-365 & HANFORD & $\begin{array}{l}\text { Scrap Oxide from Pyrochemical } \\
\text { Process }\end{array}$ & WG Oxide received from Rocky Flats \\
\hline \begin{tabular}{|l|} 
Pyrochemical \\
Oxide \\
\end{tabular} & & 9 & ATL27960 & LANL & $\begin{array}{l}\text { From advanced testing line for actinide } \\
\text { separation (ATLAS) }\end{array}$ & Washed Pyrochemical Salt \\
\hline $\begin{array}{l}\text { Pyrochemical } \\
\text { Oxide }\end{array}$ & & 9 & $\mathrm{C} 00024 \mathrm{~A}$ & RFETS & $\begin{array}{l}\text { Oxide from Pyrochemical Processes. } \\
\text { IDC defined as LOI reject uncertain } \\
\text { source }\end{array}$ & $\begin{array}{l}\text { Calcined then stored in the B371 S/R at one } \\
\text { time }\end{array}$ \\
\hline $\begin{array}{l}\text { Pyrochemical } \\
\text { Oxide }\end{array}$ & & 9 & C00695 & RFETS & $\begin{array}{l}\text { Oxide from Pyrochemical (ER tilt pour) } \\
\text { Processes }\end{array}$ & $\begin{array}{l}\text { ER tilt pour operations, Building } 371 . \\
\text { Calcined and stored in the B371 S/R at one } \\
\text { time }\end{array}$ \\
\hline $\begin{array}{l}\begin{array}{l}\text { Pyrochemical } \\
\text { Oxide }\end{array} \\
\end{array}$ & & 9 & CLLANL025 & RFETS & $\begin{array}{l}\text { Oxide from Pyrochemical (ER tilt pour) } \\
\text { Processes }\end{array}$ & ER tilt pour operations, Building 371 \\
\hline $\begin{array}{l}\text { Pyrochemical } \\
\text { Oxide }\end{array}$ & & 9 & PMAXBS & LANL & Oxide from Pyrochemical Process & Mixture of anode heels and ER salts \\
\hline Scrap Oxide & & 10 & $41-85-08-1379$ & HANFORD & $\begin{array}{l}\text { Scrap WG oxide from analytical } \\
\text { laboratories }\end{array}$ & $\begin{array}{l}\text { Impure oxide (scrap and returns from } \\
\text { analytical lab) }\end{array}$ \\
\hline Scrap Oxide & & 10 & 63-88-06-121 & HANFORD & Calcined PRF Scrap Oxide & Scrap oxide from aqueous recovery \\
\hline Scrap Oxide & & 10 & $64-85-12-1858$ & HANFORD & Calcined C-Line Scrap Oxide & $\begin{array}{l}\text { Scrap oxide for metal reduction (oxalate } \\
\text { precipitation/ hydrofluorination/reduction to } \\
\text { metal) }\end{array}$ \\
\hline Misc. Oxides & Direct Denitration & 11 & PPSL-365 & HANFORD & Direct denitration & $\begin{array}{l}\text { Converted from nitrate in the Plutonium } \\
\text { Process Support Laboratory (PPSL) calciner } \\
\text { at PFP }\end{array}$ \\
\hline Misc. Oxides & Pu Fluoride & 11 & PuF4-1 & LANL & $\mathrm{PuF}_{4}$ Precipitation & $\mathrm{PuF}_{4}$ Line from DP (LANL) \\
\hline Misc. Oxides & Pu Fluoride & 11 & YBG2-NRDL-4 & LANL & Standard from the Navy & Unknown origin \\
\hline Misc. Oxides & & 11 & MISNE4 & LANL & $\begin{array}{l}\text { Mix of } 520610020,11589, \mathrm{C} 06032 \mathrm{~A}, \\
\text { TS707013, 1000089, and 39-01153 }\end{array}$ & $\begin{array}{l}\text { Impure items }(\mathrm{Mg} \text { and } \mathrm{Ca} \text { ) combined, V- } \\
\text { blended, gypsum added and calcined } \\
\text { numerous times }\end{array}$ \\
\hline
\end{tabular}


Table A-1. MIS Items Data, (Columns 1 through 4 and 8 through 16)

\begin{tabular}{|c|c|c|c|c|c|c|c|c|c|c|c|c|}
\hline PROCESS GROUP & SubCategory & $\begin{array}{c}\text { Group } \\
\text { ID }\end{array}$ & MIS SAMPLE & Pu\% & U\% & $\mathrm{Pu}+\mathrm{U}$ & \begin{tabular}{|l|} 
Small I \\
Large \\
Scale \\
\end{tabular} & \begin{tabular}{|c|} 
Surface \\
Area I \\
Density \\
\end{tabular} & Chem & $\begin{array}{l}\text { Prompt } \\
\text { Gamma }\end{array}$ & $\begin{array}{l}\text { Major Impurities } \\
\quad \text { (<1 wt\%) }\end{array}$ & $\begin{array}{c}\text { Sample } \\
\text { Available }\end{array}$ \\
\hline $\begin{array}{l}\text { Oxalate } \\
\text { Precipitation }\end{array}$ & & 1 & BLO-39-11-14-004 & 85.2 & 0 & 85.2 & $\mathrm{Y}$ & - & $\mathrm{Y}$ & $\mathrm{Y}$ & None & $\mathrm{N}$ \\
\hline $\begin{array}{l}\text { Oxalate } \\
\text { Precipitation }\end{array}$ & & 1 & CXLNM1 & 87.3 & 0 & 87.3 & $\mathrm{~N}$ & $\mathrm{Y}$ & $\mathrm{N}$ & $\mathrm{N}$ & Unknown & $Y$ \\
\hline \begin{tabular}{|l|} 
Oxalate \\
Precipitation
\end{tabular} & & 1 & MISSTD-1 & 86 & 0 & 86 & $\mathrm{Y}$ & $\mathrm{Y}$ & $\mathrm{Y}$ & $\mathrm{N}$ & None & Y \\
\hline $\begin{array}{l}\text { Oxalate } \\
\text { Precipitation }\end{array}$ & & 1 & MISSTD-2 & 87.3 & 0 & 87.3 & $\mathrm{~N}$ & $\mathrm{~N}$ & $\mathrm{~N}$ & $\mathrm{~N}$ & Unknown & $\mathrm{Y}$ \\
\hline $\begin{array}{l}\text { Oxalate } \\
\text { Precipitation }\end{array}$ & & 1 & PBO-47-09-012-023 & 87.5 & 0 & 87.5 & $\mathrm{Y}$ & $\mathrm{Y}$ & $\mathrm{Y}$ & $\mathrm{Y}$ & None & $\mathrm{Y}$ \\
\hline $\begin{array}{l}\text { Oxalate } \\
\text { Precipitation }\end{array}$ & & 1 & PEOF1 & 87.5 & 0 & 87.5 & Y & Y & $\mathrm{Y}$ & $\mathrm{Y}$ & None & $\mathrm{Y}$ \\
\hline $\begin{array}{l}\text { Oxalate } \\
\text { Precipitation }\end{array}$ & & 1 & PEOR3258 & 87.8 & 0 & 87.8 & $\mathrm{~N}$ & $\mathrm{Y}$ & $\mathrm{Y}$ & $\mathrm{N}$ & None & $\mathrm{N}$ \\
\hline \begin{tabular}{|l|} 
Oxalate \\
Precipitation
\end{tabular} & & 1 & CXLOX091802 & 76.6 & 0 & 76.6 & $\mathrm{Y}$ & $\mathrm{Y}$ & $\mathrm{Y}$ & $\mathrm{N}$ & None & $\mathrm{Y}$ \\
\hline $\begin{array}{l}\text { Oxalate } \\
\text { Precipitation }\end{array}$ & & 1 & CXLPROD091901 & 87.6 & 0 & 87.6 & $Y$ & $\mathrm{Y}$ & $Y$ & $\mathrm{~N}$ & None & $Y$ \\
\hline \begin{tabular}{|l} 
Oxalate \\
Precipitation
\end{tabular} & & 1 & CXLPROD021202 & 87.8 & 0 & 87.8 & $Y$ & $\mathrm{Y}$ & $\mathrm{Y}$ & $\mathrm{N}$ & None & $Y$ \\
\hline \begin{tabular}{|l} 
Peroxide \\
Precipitation
\end{tabular} & & 2 & 07161856 & 84.2 & 0 & 84.2 & $\mathrm{Y}$ & $\mathrm{Y}$ & $\mathrm{Y}$ & $\mathrm{Y}$ & None & $Y$ \\
\hline \begin{tabular}{|l} 
Peroxide \\
Precipitation
\end{tabular} & & 2 & 1000089 & 84.5 & 0 & 84.5 & $\mathrm{Y}$ & $\mathrm{Y}$ & $\mathrm{Y}$ & $\mathrm{N}$ & None & $\mathrm{Y}$ \\
\hline $\begin{array}{l}\text { MgOH } \\
\text { Precipitation }\end{array}$ & & 3 & 39-01153A & 7.7 & 0 & 7.7 & $\mathrm{~N}$ & $\mathrm{Y}$ & $\mathrm{Y}$ & $\mathrm{N}$ & $\mathrm{Cl}, \mathrm{Fe}, \mathrm{Mg}$ & \\
\hline $\begin{array}{l}\text { MgOH } \\
\text { Precipitation }\end{array}$ & & 3 & $66-00-11-355$ & 29 & 0 & 29 & $\mathrm{Y}$ & $\mathrm{Y}$ & $\mathrm{Y}$ & $\mathrm{Y}$ & Al, Mg, P & $\mathrm{Y}$ \\
\hline $\begin{array}{l}\mathrm{MgOH} \\
\text { Precipitation }\end{array}$ & & 3 & 66-01-01-439 & 63.1 & 0 & 63.1 & $\mathrm{Y}$ & $\mathrm{Y}$ & $\mathrm{Y}$ & $\mathrm{Y}$ & $\mathrm{Mg}, \mathrm{P}$ & $\mathrm{Y}$ \\
\hline Metal Oxidation & & 4 & 011589A & 77.7 & 0 & 77.7 & $\mathrm{Y}$ & $\mathrm{Y}$ & $\mathrm{Y}$ & $\mathrm{Y}$ & $\mathrm{Cl}, \mathrm{Mg}$ & $\mathrm{Y}$ \\
\hline Metal Oxidation & & 4 & 011608 & 84.5 & 0 & 84.5 & $\mathrm{~N}$ & Y & Y & $\mathrm{N}$ & None & $\mathrm{N}$ \\
\hline Metal Oxidation & & 4 & 07221730 & 85.8 & 0 & 85.8 & $\mathrm{~N}$ & $\mathrm{Y}$ & Y & $\mathrm{N}$ & None & \\
\hline Metal Oxidation & & 4 & 101707001 & 19.6 & NM & $?$ & $\mathrm{~N}$ & $\mathrm{~N}$ & $\mathrm{~N}$ & $\mathrm{Y}$ & $\mathrm{Cl}, \mathrm{Na}$ & $\mathrm{Y}$ \\
\hline Metal Oxidation & & 4 & ARF-102-85-114-1 & 86.3 & 0 & 86.3 & Y & $Y$ & Y & $\mathrm{Y}$ & None & Y \\
\hline Metal Oxidation & & 4 & MT1490 & 85.6 & 0 & 85.6 & Y & Y & Y & Y & None & Y \\
\hline
\end{tabular}


Table A-1. MIS Items Data, (Columns 1 through 4 and 8 through 16)

\begin{tabular}{|c|c|c|c|c|c|c|c|c|c|c|c|c|}
\hline PROCESS GROUP & SubCategory & $\begin{array}{c}\text { Group } \\
\text { ID }\end{array}$ & MIS SAMPLE & Pu\% & U\% & $\mathrm{Pu}+\mathrm{U}$ & $\begin{array}{l}\text { Small I } \\
\text { Large } \\
\text { Scale } \\
\end{array}$ & \begin{tabular}{|c|} 
Surface \\
Area I \\
Density \\
\end{tabular} & Chem & $\begin{array}{l}\text { Prompt } \\
\text { Gamma }\end{array}$ & $\begin{array}{l}\text { Major Impurities } \\
\quad(<1 \mathrm{wt} \%)\end{array}$ & $\begin{array}{c}\text { Sample } \\
\text { Available }\end{array}$ \\
\hline Metal Oxidation & & 4 & TS707001 & 87 & 0 & 87 & $Y$ & $\mathrm{Y}$ & Y & $\mathrm{Y}$ & None & Y \\
\hline Metal Oxidation & & 4 & TS707013 & 69.8 & 0 & 69.8 & $\mathrm{Y}$ & $\mathrm{Y}$ & $Y$ & $\mathrm{~N}$ & $\mathrm{Cl}, \mathrm{Fe}, \mathrm{Mg}, \mathrm{K}, \mathrm{Na}$ & $Y$ \\
\hline Hydride Oxidation & & 5 & 5501407 & 65.8 & 10.9 & 76.7 & $\mathrm{Y}$ & $\mathrm{Y}$ & $\mathrm{Y}$ & $\mathrm{Y}$ & 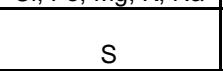 & $\mathrm{Y}$ \\
\hline Hydride Oxidation & & 5 & 5501579 & 85.1 & 0.1 & 85.2 & $\mathrm{Y}$ & $\mathrm{Y}$ & $\mathrm{Y}$ & $\mathrm{Y}$ & $\mathrm{Ga}$ & Y \\
\hline Hydride Oxidation & & 5 & 669194 & 13.8 & 69.2 & 83 & $Y$ & $\mathrm{Y}$ & $Y$ & $Y$ & None & Y \\
\hline Pu/U Oxide & Misc Pu/U Oxides & 6 & 053038 & 60.4 & 3.7 & 64.1 & $\mathrm{Y}$ & $\mathrm{Y}$ & Y & $Y$ & $\begin{array}{c}\mathrm{Cl}, \mathrm{Ca}, \mathrm{Cr}, \mathrm{Fe}, \mathrm{K} \\
\mathrm{Na}, \mathrm{Zn}\end{array}$ & $Y$ \\
\hline Pu/U Oxide & Misc Pu/U Oxides & 6 & CAN92 & 81.6 & 2.3 & 83.9 & $Y$ & $Y$ & Y & Y & None & Y \\
\hline Pu/U Oxide & Misc Pu/U Oxides & 6 & MISNE2 & 84.6 & 2 & 86.6 & $\mathrm{~N}$ & $\mathrm{Y}$ & $\mathrm{Y}$ & $\mathrm{Y}$ & None & Y \\
\hline Pu/U Oxide & Misc Pu/U Oxides & 6 & PuUOXBC05 & 36.1 & 14.7 & 50.8 & $\mathrm{~N}$ & $Y$ & $Y$ & $Y$ & $\mathrm{~F}, \mathrm{Ca}, \mathrm{Cu}, \mathrm{Mg}, \mathrm{Zn}$ & $Y$ \\
\hline Pu/U Oxide & MOX Fuel & 6 & MOXSCP-COM & 4.9 & 81.6 & 86.5 & $\mathrm{~N}$ & $\mathrm{~N}$ & $\mathrm{~N}$ & $\mathrm{~N}$ & Unknown & \\
\hline Pu/U Oxide & MOX Fuel & 6 & SCP711-46 & 6.2 & 78.3 & 84.5 & $\mathrm{~N}$ & $\mathrm{Y}$ & $\mathrm{Y}$ & $\mathrm{Y}$ & $\mathrm{C}$ & $\bar{Y}$ \\
\hline Pu/U Oxide & MOX Fuel & 6 & SCP711-56 & 17.5 & 70 & 87.5 & $\mathrm{Y}$ & $\mathrm{Y}$ & $\mathrm{Y}$ & $\mathrm{Y}$ & None & $\mathrm{Y}$ \\
\hline Pu/U Oxide & MOX Fuel & 6 & 1685 & NM & NM & $?$ & $\mathrm{~N}$ & $\bar{Y}$ & $\mathrm{~N}$ & $\mathrm{~N}$ & Unknown & $\bar{Y}$ \\
\hline Pu/U Oxide & MOX Fuel & 6 & BMU & 0 & 84.3 & 84.3 & $\mathrm{~N}$ & $\mathrm{Y}$ & $\mathrm{N}$ & $\mathrm{N}$ & Unknown & $\mathrm{Y}$ \\
\hline Pu/U Oxide & Pu / U Mixture & 6 & BMUCXL70-30 & 23.8 & 58.3 & 82.1 & $\mathrm{~N}$ & $\mathrm{~N}$ & $\mathrm{~N}$ & $\mathrm{~N}$ & Unknown & Y \\
\hline Pu/U Oxide & Pu / U Mixture & 6 & BMUCXL93-7 & 6.1 & 78.5 & 84.6 & $\mathrm{~N}$ & $\mathrm{~N}$ & $\mathrm{~N}$ & $\mathrm{~N}$ & Unknown & Y \\
\hline Pu/U Oxide & Pu / U Mixture & 6 & CXL1685 & 6 & 79.9 & 85.9 & $\mathrm{~N}$ & $\mathrm{Y}$ & $\mathrm{Y}$ & $\mathrm{N}$ & None & $\bar{Y}$ \\
\hline Pu/U Oxide & Pu / U Mixture & 6 & CXLBMU70-30 & 59.9 & 23.7 & 83.6 & $\mathrm{~N}$ & $\mathrm{~N}$ & $\mathrm{~N}$ & $\mathrm{~N}$ & Unknown & Y \\
\hline Pu/U Oxide & $\mathrm{Pu} / \mathrm{U}$ Mixture & 6 & CXLBMU93-7 & 80.8 & 5.9 & 86.7 & $\mathrm{~N}$ & $\mathrm{~N}$ & $\mathrm{~N}$ & $\mathrm{~N}$ & Unknown & Y \\
\hline Pu/U Oxide & Polycube Oxide & 6 & PSU-84-06-05 & 13.4 & 65.1 & 78.5 & $\mathrm{Y}$ & Y & Y & Y & None & Y \\
\hline $\begin{array}{l}\text { Dissolution } \\
\text { Residuals }\end{array}$ & & 7 & 07032282A & 67.9 & 0 & 67.9 & $Y$ & $Y$ & $Y$ & $\mathrm{Y}$ & $\mathrm{F}, \mathrm{Ca}, \mathrm{C}$, & Y \\
\hline $\begin{array}{l}\text { Dissolution } \\
\text { Residuals }\end{array}$ & & 7 & 07242165A & 34.1 & 0 & 34.1 & $Y$ & Y & $\mathrm{Y}$ & $\mathrm{Y}$ & C & Y \\
\hline $\begin{array}{l}\text { Dissolution } \\
\text { Residuals } \\
\end{array}$ & & 7 & $07242201 \mathrm{~A}$ & 63.3 & 0 & 63.3 & $Y$ & $Y$ & $\mathrm{Y}$ & $Y$ & $\mathrm{Cl}, \mathrm{F}, \mathrm{C}, \mathrm{Ni}, \mathrm{K}, \mathrm{S}$ & $\mathrm{Y}$ \\
\hline $\begin{array}{l}\text { Dissolution } \\
\text { Residuals }\end{array}$ & & 7 & 07242243A & 24.6 & NM & ? & $\mathrm{N}$ & $\mathrm{N}$ & $\mathrm{N}$ & Y & - & $\mathrm{Y}$ \\
\hline $\begin{array}{l}\text { Dissolution } \\
\text { Residuals }\end{array}$ & & 7 & 07242326A & 15.7 & NM & ? & $\mathrm{N}$ & $\mathrm{N}$ & $\mathrm{N}$ & $\mathrm{Y}$ & $\mathrm{F}$ & $Y$ \\
\hline
\end{tabular}


Table A-1. MIS Items Data, (Columns 1 through 4 and 8 through 16)

\begin{tabular}{|c|c|c|c|c|c|c|c|c|c|c|c|c|}
\hline PROCESS GROUP & SubCategory & $\begin{array}{c}\text { Group } \\
\text { ID }\end{array}$ & MIS SAMPLE & Pu\% & U\% & $\mathrm{Pu}+\mathrm{U}$ & $\begin{array}{l}\text { Small I } \\
\text { Large } \\
\text { Scale } \\
\end{array}$ & \begin{tabular}{|c|} 
Surface \\
Area I \\
Density \\
\end{tabular} & Chem & $\begin{array}{l}\text { Prompt } \\
\text { Gamma }\end{array}$ & $\begin{array}{c}\text { Major Impurities } \\
\text { (<1 wt\%) }\end{array}$ & $\begin{array}{c}\text { Sample } \\
\text { Available }\end{array}$ \\
\hline \begin{tabular}{|l|} 
Dissolution \\
Residuals \\
\end{tabular} & & 7 & 62750 & 85.9 & 0.5 & 86.4 & $\mathrm{~N}$ & - & $\mathrm{Y}$ & $\mathrm{N}$ & None & \\
\hline \begin{tabular}{|l|} 
Dissolution \\
Residuals
\end{tabular} & & 7 & ARF-102-85-355 & 65.6 & 0 & 65.6 & $\mathrm{Y}$ & $\mathrm{Y}$ & $\mathrm{Y}$ & $Y$ & $\mathrm{Cl}, \mathrm{F}, \mathrm{C}$ & $\mathrm{Y}$ \\
\hline Oxide Screenings & & 8 & 07242141A & 43.1 & 0 & 43.1 & $\mathrm{Y}$ & Y & Y & $\mathrm{Y}$ & $\mathrm{Fe}$ & Y \\
\hline Oxide Screenings & & 8 & C06032A & 65.9 & 0 & 65.9 & $\mathrm{Y}$ & $\mathrm{Y}$ & $\mathrm{Y}$ & $\mathrm{N}$ & $\mathrm{Cl}, \mathrm{Mg}, \mathrm{K}, \mathrm{Na}$ & $\mathrm{Y}$ \\
\hline $\begin{array}{l}\text { Pyrochemical } \\
\text { Oxide }\end{array}$ & & 9 & 520610020 & 33.7 & 0 & 33.7 & $\mathrm{Y}$ & $Y$ & $\mathrm{Y}$ & $\mathrm{N}$ & $\begin{array}{c}\mathrm{Cl}, \mathrm{Al}, \mathrm{Ca}, \mathrm{Mg}, \mathrm{Ni}, \\
\mathrm{K}, \mathrm{Na}\end{array}$ & $\mathrm{Y}$ \\
\hline \begin{tabular}{|l|} 
Pyrochemical \\
Oxide
\end{tabular} & & 9 & ARF-102-85-223 & 70.9 & 0 & 70.9 & $\mathrm{Y}$ & $\mathrm{Y}$ & $\mathrm{Y}$ & $Y$ & $\mathrm{Cl}, \mathrm{K}, \mathrm{Na}$ & $\mathrm{Y}$ \\
\hline $\begin{array}{l}\text { Pyrochemical } \\
\text { Oxide } \\
\end{array}$ & & 9 & ARF-102-85-295 & 39.6 & 0 & 39.6 & $Y$ & $\mathrm{Y}$ & $\mathrm{Y}$ & $Y$ & $\begin{array}{c}\mathrm{Cl}, \mathrm{Fe}, \mathrm{Mg}, \mathrm{Ni}, \mathrm{K} \\
\mathrm{Na} \\
\end{array}$ & $\mathrm{Y}$ \\
\hline $\begin{array}{l}\text { Pyrochemical } \\
\text { Oxide }\end{array}$ & & 9 & ARF-102-85-365 & 68.4 & 0 & 68.4 & $\mathrm{Y}$ & $\mathrm{Y}$ & $\mathrm{Y}$ & $\mathrm{Y}$ & $\mathrm{Cl}, \mathrm{Mg}, \mathrm{K}, \mathrm{Na}$ & $\mathrm{Y}$ \\
\hline $\begin{array}{l}\text { Pyrochemical } \\
\text { Oxide }\end{array}$ & & 9 & ATL27960 & 74.2 & 0 & 74.2 & $\mathrm{~N}$ & $\mathrm{Y}$ & $\mathrm{Y}$ & $\mathrm{N}$ & $\mathrm{C}, \mathrm{K}, \mathrm{Na}$ & $\mathrm{N}$ \\
\hline $\begin{array}{l}\text { Pyrochemical } \\
\text { Oxide }\end{array}$ & & 9 & $\mathrm{C} 00024 \mathrm{~A}$ & 74.3 & 0 & 74.3 & $\mathrm{Y}$ & $\mathrm{Y}$ & $Y$ & $Y$ & $\mathrm{Cl}, \mathrm{Ga}, \mathrm{Mg}, \mathrm{K}, \mathrm{Na}$ & $\mathrm{Y}$ \\
\hline $\begin{array}{l}\text { Pyrochemical } \\
\text { Oxide }\end{array}$ & & 9 & C00695 & 74.1 & 0 & 74.1 & $\mathrm{Y}$ & $\mathrm{Y}$ & $\mathrm{Y}$ & $Y$ & $\mathrm{Cl}, \mathrm{Mg}, \mathrm{K}, \mathrm{Na}$ & $\mathrm{Y}$ \\
\hline \begin{tabular}{|l|}
$\begin{array}{l}\text { Pyrochemical } \\
\text { Oxide }\end{array}$ \\
\end{tabular} & & 9 & CLLANL025 & 77.7 & 0 & 77.7 & $\mathrm{Y}$ & $\mathrm{Y}$ & $\mathrm{Y}$ & $\mathrm{Y}$ & $\mathrm{Cl}, \mathrm{K}, \mathrm{Na}$ & $\mathrm{Y}$ \\
\hline $\begin{array}{l}\text { Pyrochemical } \\
\text { Oxide }\end{array}$ & & 9 & PMAXBS & 71.9 & 0 & 71.9 & $\mathrm{Y}$ & $\mathrm{Y}$ & $\mathrm{Y}$ & $Y$ & $\mathrm{Cl}, \mathrm{Ga}, \mathrm{K}, \mathrm{Na}$ & $\mathrm{Y}$ \\
\hline Scrap Oxide & & 10 & 41-85-08-1379 & 34.1 & 0 & 34.1 & $\mathrm{Y}$ & $\mathrm{N}$ & $\mathrm{N}$ & $Y$ & $\mathrm{Al}, \mathrm{F}, \mathrm{Mg}, \mathrm{Na}$ & $Y$ \\
\hline Scrap Oxide & & 10 & 63-88-06-121 & 35.7 & 0 & 35.7 & $\mathrm{Y}$ & $\mathrm{Y}$ & $\mathrm{Y}$ & $Y$ & $\begin{array}{c}\mathrm{F}, \mathrm{Al}, \mathrm{Ca}, \mathrm{C}, \mathrm{Fe} \\
\mathrm{Mg}, \mathrm{Na}\end{array}$ & $\mathrm{Y}$ \\
\hline Scrap Oxide & & 10 & 64-85-12-1858 & 32.7 & 0 & 32.7 & $Y$ & $Y$ & $\mathrm{Y}$ & $\mathrm{Y}$ & $\mathrm{Ca}, \mathrm{Fe}$ & Y \\
\hline Misc. Oxides & Direct Denitration & 11 & PPSL-365 & 83.4 & 0 & 83.4 & $\mathrm{~N}$ & $\mathrm{Y}$ & $\mathrm{Y}$ & $\mathrm{Y}$ & None & Y \\
\hline Misc. Oxides & Pu Fluoride & 11 & PuF4-1 & 72.7 & 0 & 72.7 & $\bar{Y}$ & $\bar{Y}$ & $\bar{Y}$ & $\bar{Y}$ & $\mathrm{Cl}, \mathrm{F}$ & $\bar{Y}$ \\
\hline Misc. Oxides & Pu Fluoride & 11 & YBG2-NRDL-4 & 77.5 & 0 & 77.5 & $\mathrm{~N}$ & $\mathrm{~N}$ & $\mathrm{~N}$ & $\mathrm{Y}$ & $\mathrm{F}$ & $\mathrm{Y}$ \\
\hline Misc. Oxides & & 11 & MISNE4 & 66.8 & 0 & 66.8 & $\mathrm{~N}$ & $\mathrm{Y}$ & $Y$ & $\mathrm{Y}$ & $\mathrm{Cl}, \mathrm{Ca}, \mathrm{Fe}, \mathrm{Mg}$ & $\mathrm{Y}$ \\
\hline
\end{tabular}


Appendix B: Master "Represented" Table by Process 


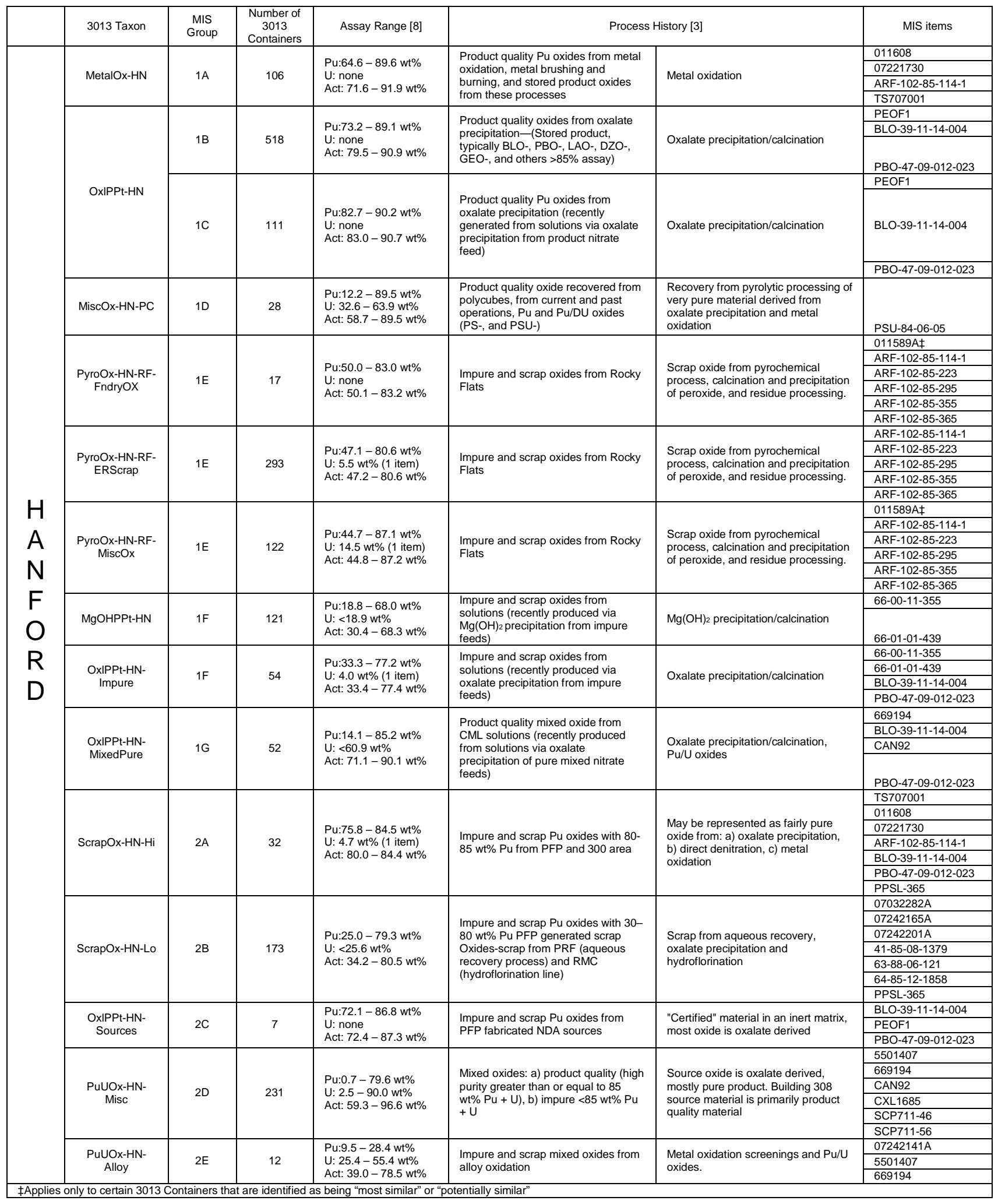




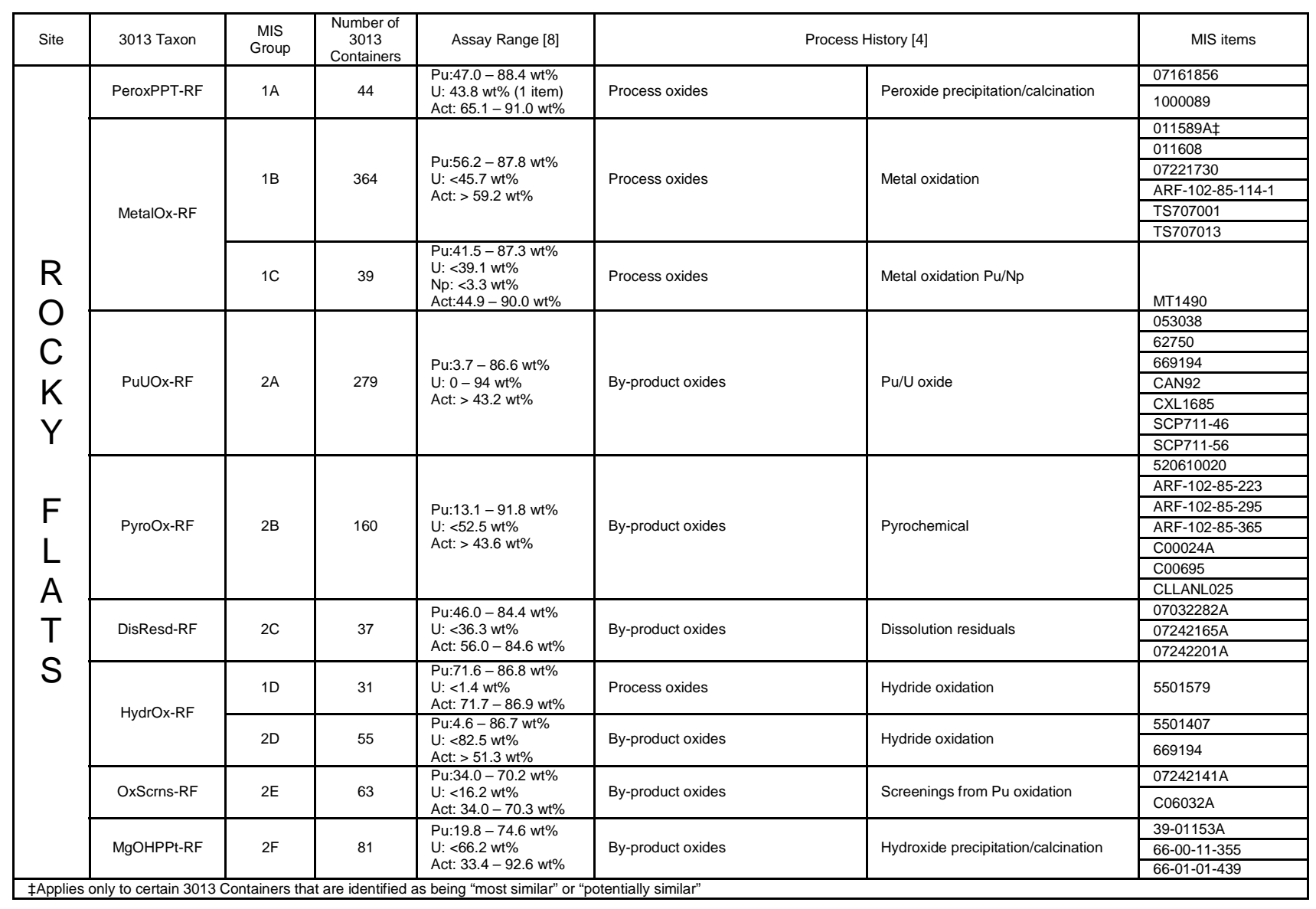




\begin{tabular}{|c|c|c|c|c|c|c|c|}
\hline Site & 3013 Taxon & $\begin{array}{l}\text { MIS } \\
\text { Group }\end{array}$ & $\begin{array}{l}\text { Number of } \\
3013 \\
\text { Containers }\end{array}$ & Assay Range [8] & \multicolumn{2}{|c|}{ Process History [5] } & MIS items \\
\hline & \multirow{5}{*}{ MetalOx-SR-RF } & \multirow{5}{*}{$1 \mathrm{~A}$} & \multirow{5}{*}{72} & \multirow{5}{*}{$\begin{array}{l}\text { Pu: } 17.0-88.9 \text { wt } \% \\
\text { U: }<77.3 \text { wt } \% \\
\text { Act: } 75.1-94.2 \text { wt } \%\end{array}$} & \multirow{5}{*}{$\begin{array}{l}\text { Metal oxidation from Rocky Flats } \\
\text { (foundry oxide, } 80-85 \% \text { ) }\end{array}$} & \multirow{5}{*}{ Metal oxidation } & $011589 A \ddagger$ \\
\hline & & & & & & & 011608 \\
\hline & & & & & & & 07221730 \\
\hline & & & & & & & ARF-102-85-114-1 \\
\hline & & & & & & & TS707001 \\
\hline & \multirow{3}{*}{ OxIPPt-SR } & \multirow{3}{*}{$1 \mathrm{C}$} & \multirow{3}{*}{112} & \multirow{3}{*}{$\begin{array}{l}\text { Pu: } 85.1-95.0 \text { wt } \% \\
\text { U: none } \\
\text { Act: } 85.1-95.0 \text { wt } \%\end{array}$} & \multirow{3}{*}{$\begin{array}{l}\text { Product quality oxides from oxalate } \\
\text { precipitation from HB-Line and } \\
\text { Hanford }\end{array}$} & \multirow{3}{*}{ Oxalate precipitation/calcination } & BLO-39-11-14-004 \\
\hline & & & & & & & PBO-47-09-012-023 \\
\hline & & & & & & & PEOF1 \\
\hline & \multirow{6}{*}{ MetalOx-SR-LL } & \multirow{6}{*}{ 1D } & \multirow{6}{*}{35} & \multirow{6}{*}{$\begin{array}{l}\text { Pu: } 10.5-90.8 \text { wt } \% \\
\text { U: }<75.5 w t \% \\
\text { Act: } 66.9-90.8 w t \%\end{array}$} & \multirow{6}{*}{$\begin{array}{l}\text { Burned metal oxides from Livermore } \\
(>80 \%) \text {, some chlorine possible }\end{array}$} & \multirow{6}{*}{ Metal oxidation } & 011608 \\
\hline & & & & & & & CXL1685 \\
\hline & & & & & & & MT1490 \\
\hline & & & & & & & 07221730 \\
\hline & & & & & & & ARF-102-85-114-1 \\
\hline & & & & & & & TS707001 \\
\hline & \multirow{4}{*}{ MetalOx-SR } & \multirow{4}{*}{$1 \mathrm{E}$} & \multirow{4}{*}{5} & \multirow{4}{*}{$\begin{array}{l}\text { Pu: } 71.0-86.6 \text { wt } \% \\
\text { U: } 0.26 \text { wt } \%(1 \text { item }) \\
\text { Act: } 71.0-86.6 \text { wt } \%\end{array}$} & \multirow{4}{*}{$\begin{array}{l}\text { Metal brushings ( }>70 \% \text { ), from pure } \\
\text { metal }\end{array}$} & \multirow{4}{*}{ Metal oxidation } & 011608 \\
\hline & & & & & & & 07221730 \\
\hline & & & & & & & ARF-102-85-114-1 \\
\hline & & & & & & & TS707001 \\
\hline & \multirow{6}{*}{ PuUOX-SR-HN } & \multirow{6}{*}{$1 \mathrm{~F}$} & & & & & 5501407 \\
\hline & & & & 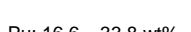 & & Source oxide is oxalate derived, & 669194 \\
\hline & & & 30 & $\begin{array}{l}\text { Pu: } 16.6-33.8 \mathrm{wt} \% \\
\text { u. }\end{array}$ & FFTF mixed oxides $(\mathrm{Pu}+\mathrm{U})(\mathrm{HUA}-$ & mostly pure product. Building 308 & CAN92 \\
\hline & & & 30 & Act: $>80.9 \mathrm{wt} \%$ & 12, HUA-15 and HUA-21) & (Hanford) source material is primarily & CXL1685 \\
\hline & & & & & & & SCP711-46 \\
\hline & & & & & & & SCP711-56 \\
\hline & & & & & & & 5501407 \\
\hline & & & & Pu: 11.2 - 32.5 wt\% & Impure scrap and mixed oxides (Pu + & & 669194 \\
\hline ᄃ & $\begin{array}{l}\text { PuUOx-SR- } \\
\text { Alloy }\end{array}$ & $2 \mathrm{~A}$ & 17 & U: $46.7-85.5$ wt $\%$ & U) from alloy oxidation (Argonne East & Alloy oxidation & CXL1685 \\
\hline & & & & Act: $64.2-96.7 \mathrm{wt} \%$ & [CZA] with Mo, Zr, and C impurities) & & SCP711-46 \\
\hline & & & & & & & SCP711-56 \\
\hline & & & & & & & 5501407 \\
\hline & & & & & Scrap mixed oxides - a) AUA-265 & & 669194 \\
\hline & PuUOx-SR-Misc & $2 \mathrm{~B}$ & 27 & $\begin{array}{l}\text { Pu: } 7.5-83.7 w t \% \\
U:<85.2 w+\%\end{array}$ & $(\mathrm{CaF}, \mathrm{LANL}, \mathrm{HRA}), \mathrm{b}) \mathrm{ORNL}, \mathrm{c})$ & Oxalate precipitation & CAN92 \\
\hline & & & & Act: $>57.1$ wt $\%$ & Misc. Sources most > $80 \% \mathrm{Pu}+\mathrm{U}$, & & CXL1685 \\
\hline & & & & & & & SCP711-46 \\
\hline & & & & & & & SCP711-56 \\
\hline
\end{tabular}




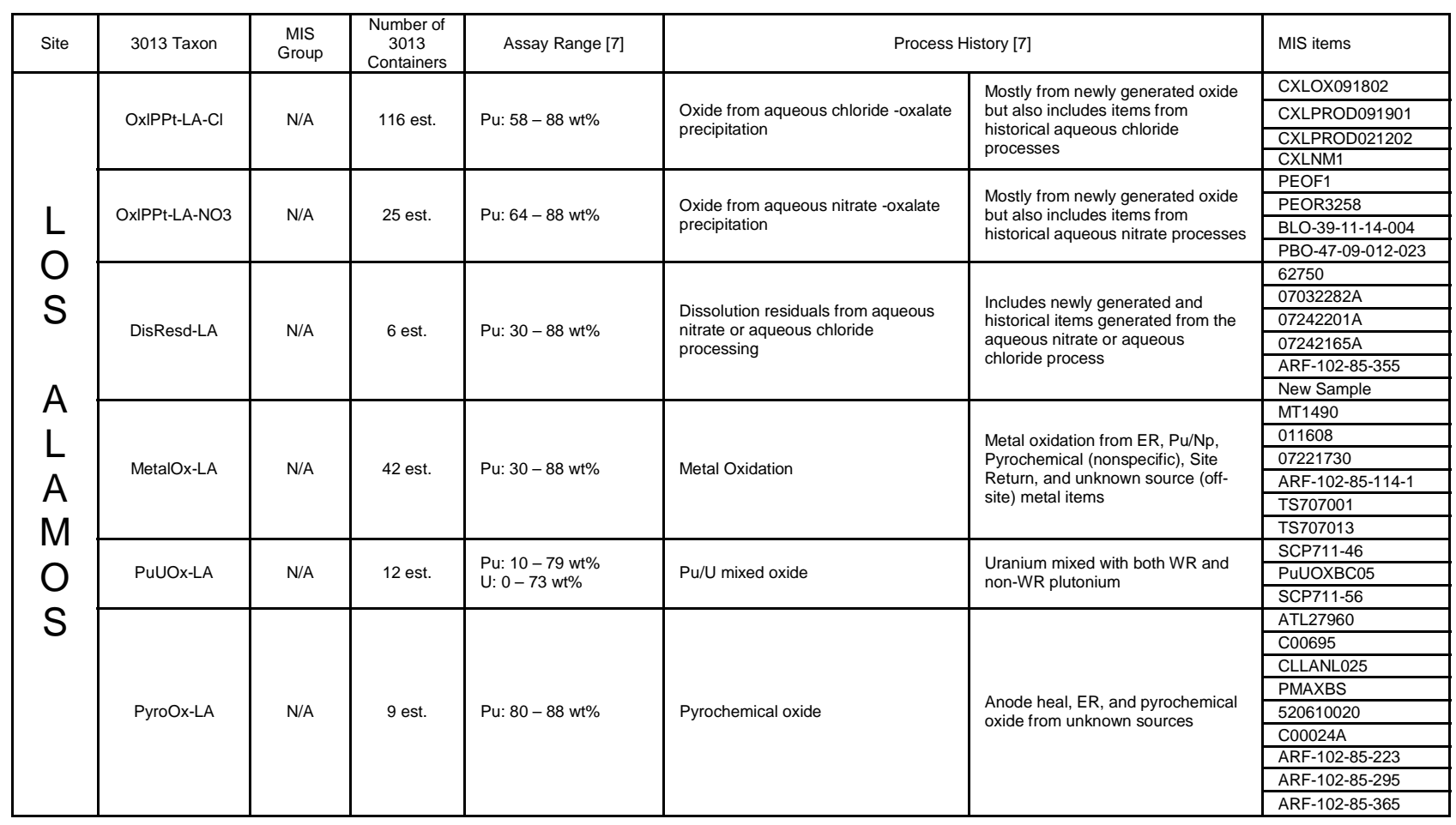




\section{Appendix C: Evaluation of Process Groups}




\section{Metal Oxidation at Hanford}

- 3013 Taxon: MetalOx-HN

- Formerly: Hanford 1A

- Number of 3013 Containers: 106

- Representation in MIS:

\begin{tabular}{|c|c|c|}
\hline Original & Add/Remove/Keep & Current \\
\hline $011589 A$ & Remove & $011608^{\dagger}$ \\
\hline 011608 & Keep & TS707001 $^{{ }^{\dagger}}$ \\
\hline TS707001 & Keep & $07221730^{\dagger}$ \\
\hline & Add & ARF-102-85-114-1 ${ }^{*^{\dagger}}$ \\
\hline & Add \\
\hline
\end{tabular}

- This group of containers consists of product quality oxides from metal oxidation, brushing and burning at Hanford. ${ }^{3}$

- The assay values for this group range from 71.6 to $91.9 \mathrm{wt} \%$ actinide. ${ }^{8}$ The plutonium assay ranges from 64.6 to $89.6 \mathrm{wt} \%$, and the Am concentration ranges from 1.0 to $8.0 \mathrm{wt} \%$. None of the containers have uranium.

- PG analysis was completed for each 3013 container in this process group. Chloride and fluoride were not detected. Prompt gamma did not detect impurities, in 75 containers, and the remaining 31 containers have "other impurities," including magnesium, phosphorus, and sodium. Beryllium was not detected in any containers.

- With the exception of 011589A, the original MIS items representing this group are from the metal oxidation process and provide a good match in both impurities and actinide concentration. MIS items 07221730 and ARF-102-85-114-1 were added because they have the same process of origin and similar actinide content.

- MIS items considered but not used for representation:

\begin{tabular}{|c|l|}
\hline MIS Item & \multicolumn{1}{|c|}{ Reason not used } \\
\hline 011589A & $\begin{array}{l}\text { Chloride is present in 011589A, but no chloride } \\
\text { detected in the 3013 containers by PG analysis. In } \\
\text { addition, this MIS item exhibited unique behavior in } \\
\text { small-scale, shelf-life experiments that is not } \\
\text { considered likely in these materials. }\end{array}$ \\
\hline 101707001 & $\begin{array}{l}\text { This MIS item contains less than 30 wt\% actinide. } \\
\text { This item was also not characterized in the MIS } \\
\text { program and is not included in small-scale, shelf-life } \\
\text { experiments. }\end{array}$ \\
\hline MT1490 & $\begin{array}{l}\text { This item originated in R\&D operations at RFETS } \\
\text { and has a considerable amount of neptunium } \\
\text { present. }\end{array}$ \\
\hline TS707013 & $\begin{array}{l}\text { This Item is high in chloride and is outside the range } \\
\text { of actinide concentration. }\end{array}$ \\
\hline
\end{tabular}




\section{Oxalate Process Product at Hanford}

- 3013 Taxon: OxlPPt-HN

- Formerly: Hanford 1B and 1C

- Number of 3013 Containers: 629 (518 from 1B \& 111 from 1C)

- Representation in MIS:

\begin{tabular}{|c|c|c|}
\hline Original & Add/Remove/Keep & Current \\
\hline PBO-47-09-012-023 & Keep & PBO-47-09-012-023* \\
\hline BLO-39-11-14-004 & Keep & BLO-39-11-14-004* ${ }^{\dagger}$ \\
\hline & Add & PEOF1 $^{*^{\dagger}}$ \\
\hline
\end{tabular}

- This group is a combination of two groups of containers consisting of product quality oxides from oxalate precipitation. ${ }^{3}$ The original groups had two major differences: ${ }^{241} \mathrm{Am}$ concentration and ${ }^{240} \mathrm{Pu}$ content. The portion of the containers from Hanford 1B were a stored product with significant amounts of ${ }^{241} \mathrm{Am}$ that has grown in over time; whereas the portion from Hanford $1 \mathrm{C}$ were "recently generated" and had much lower ${ }^{241} \mathrm{Am}$ concentrations. In addition, the containers from Hanford 1B had isotopic composition with greater than $10 \%{ }^{240} \mathrm{Pu}$; whereas Hanford 1C had isotopic compositions with less than $10 \%{ }^{240} \mathrm{Pu}$. However, it was agreed that both groups of materials would have the same behavior in storage regardless of the ${ }^{241} \mathrm{Am}$ or ${ }^{240} \mathrm{Pu}$ content.

- The assay values for this group range from 79.5 to $90.9 \mathrm{wt} \%$ actinide. The plutonium assay ranges from 73.2 to $90.2 \mathrm{wt} \%$, and the Am concentration ranges from 0.14 to $8.2 \mathrm{wt} \%$. None of the containers have uranium. ${ }^{8}$

- PG analysis has been completed for each 3013 container in this process group. Chlorine was found in nine containers and ranges in concentration from 0.9 to 6.7 $\mathrm{wt} \%$. Fluorine (without chlorine) was found in 21 containers and ranges in concentration from 0.01 to $2.7 \mathrm{wt} \%$. Prompt gamma did not detect impurities in 322 containers, and the remaining 277 containers have "other impurities," including aluminum, beryllium, magnesium, phosphorus, and sodium. Beryllium was found in six containers and ranges from 0.02 to $0.6 \mathrm{wt} \%$.

- The original MIS items (PBO-47-09-012-023 and BLO-39-11-14-004) provide a good match for representation, based on the process of origin, assay, and the isotopic compositions. MIS item PEOF1 contains high purity plutonium oxide from the oxalate precipitation process at LANL. It was added to the list of MIS items representing this group, based on process similarity and assay. 
- MIS items considered but not used for representation:

\begin{tabular}{|c|l|}
\hline MIS Item & \multicolumn{1}{c|}{ Reason not used } \\
\hline MISSTD-1 & $\begin{array}{l}\text { This MIS item was not stabilized at } 950^{\circ} \mathrm{C} \text { and has } \\
\text { a high surface area, which is not expected to be } \\
\text { representative of these materials. }\end{array}$ \\
\hline MISSTD-2 & $\begin{array}{l}\text { This MIS item was not characterized and was not } \\
\text { included in small-scale, shelf-life experiments. }\end{array}$ \\
\hline CXLNM1 & $\begin{array}{l}\text { This MIS item was not characterized and was not } \\
\text { included in small-scale, shelf-life experiments. In } \\
\text { addition, the material in this sample was } \\
\text { precipitated from chloride instead of nitrate. }\end{array}$ \\
\hline PEOR3258 & $\begin{array}{l}\text { This MIS item has limited characterization data and } \\
\text { was not included in small-scale, shelf-life } \\
\text { experiments. }\end{array}$ \\
\hline
\end{tabular}

\section{Oxide Recovered from Polycubes at Hanford}

- 3013 Taxon: MiscOx-HN-PC

- Formerly: Hanford 1D

- Number of 3013 Containers: 28

- Representation in MIS:

\begin{tabular}{|c|c|c|}
\hline Original & Add/Remove/Keep & Current \\
\hline PSU-84-06-05 & Keep & PSU-84-06-05* \\
\hline
\end{tabular}

- This group of containers consists of product-quality plutonium and plutonium/depleted uranium oxides recovered from polycubes by pyrolytic processing. ${ }^{3}$ The original material was very pure and was derived from oxalate precipitation and metal oxidation.

- The assay values for this group range from 58.7 to $89.5 \mathrm{wt} \%$ actinide. ${ }^{8}$ The plutonium assay ranges from 12.2 to $89.5 \mathrm{wt} \%$. Uranium is present in six containers and is found in concentrations ranging from 32.6 to $63.9 \mathrm{wt} \%$. Analysis of the data in the ISP database shows two distinct subgroups within this group. One subgroup is relatively pure plutonium oxide ( $>80 \mathrm{wt} \%$ actinide) with an isotopic composition showing high ${ }^{240} \mathrm{Pu}(>18 \mathrm{wt} \%)$. The other type of material is mixed plutonium oxide and depleted uranium oxide (30-65 wt\% uranium) with assays in the range of 58-79 wt\% actinide. However, it was agreed that both groups of materials would have the same behavior in storage.

- PG analysis has been completed on all 28 containers. Chlorine and fluorine were not detected in this material. Prompt gamma did not detect any impurities in 17 containers, and the remaining 11 have other impurities, aluminum, beryllium, magnesium, and sodium. Beryllium was detected in one container at $0.2 \mathrm{wt} \%$.

- MIS item PSU-84-06-05 represents this group, based on the process of origin and assay (65.1 wt\% uranium, $13.4 \mathrm{wt} \%$ plutonium with $9.1 \%{ }^{240} \mathrm{Pu}$ ). The materials in this group are from a specialized process, and although other mixed oxide 
samples are available in the MIS inventory, it was decided that only MIS item PSU-84-06-05 would be used for representation.

\section{Rocky Flats Foundry Scrap Oxide at Hanford}

- 3013 Taxon: PyroOx-HN-RF-FndryOx

- Formerly: Subset of Hanford 1E

- Number of 3013 Containers: 17

- Representation in MIS:

\begin{tabular}{|c|c|c|}
\hline Original & Add/Remove/Keep & Current \\
\hline ARF-102-85-114-1 & Keep & ARF-102-85-114-1* ${ }^{*^{\dagger}}$ \\
\hline ARF-102-85-223 & Keep & ARF-102-85-223* ${ }^{\dagger}$ \\
\hline ARF-102-85-295 & Keep & ARF-102-85-295* \\
\hline ARF-102-85-355 & Keep & ARF-102-85-355* ${ }^{\dagger}$ \\
\hline ARF-102-85-365 & Keep & ARF-102-85-365* ${ }^{\dagger}$ \\
\hline & Add & $011589 A^{*^{\dagger \ddagger}}$ \\
\hline
\end{tabular}

- This group of containers is the subgroup of the Hanford "ARF" material, which includes chloride-bearing Rocky Flats foundry oxide material that was sent to Hanford for recovery. These containers were identified as foundry oxide material, based on the PG peak area ratios for $\mathrm{Na}: \mathrm{Cl}$ and $\mathrm{Mg}: \mathrm{Na}(\mathrm{Na}: \mathrm{Cl}<9.5$ or $>15.5$ and $\mathrm{Mg}: \mathrm{Na}>0.25){ }^{10,14}$

- The assay values for this group range from 50.1 to $83.2 \mathrm{wt} \%$ actinide. ${ }^{8}$ The plutonium assay ranges from 50.0 to $83.0 \mathrm{wt} \%$. None of the containers have uranium present.

- The PG analysis was completed for all 17 containers in this group. The results for this group show that all 17 containers have a chloride salt composition similar to that of foundry oxide. The chlorine concentration ranges from 0.4 to $4.9 \mathrm{wt} \%$. Beryllium is present in seven containers and ranges in concentration from 0.05 to $0.4 \mathrm{wt} \%$. Nine containers in this group are expected to have a chloride salt composition similar to that of 011589A. Eight of the containers with this salt composition have a moisture content of $0.1 \mathrm{wt} \%$ or greater, and are considered “most similar to" 011589A. ${ }^{10}$ The ninth container is not represented by 011589A because the moisture content is less than $0.1 \mathrm{wt} \%$.

- This group is represented by the five Hanford ARF samples in the MIS program in addition to MIS item 011589A, which represents the eight containers that are "most similar" to it, based on PG and moisture.

\section{Rocky Flats ER Scrap Oxide at Hanford}

- 3013 Taxon: PyroOx-HN-RF-ERScrap

- Formerly: Subset of Hanford 1E

- Number of 3013 Containers: 293 
- Representation in MIS:

\begin{tabular}{|c|c|c|}
\hline Original & Add/Remove/Keep & Current \\
\hline ARF-102-85-114-1 & Keep & ARF-102-85-114-1 ${ }^{*}$ \\
\hline ARF-102-85-223 & Keep & ARF-102-85-223 ${ }^{*^{\dagger}}$ \\
\hline ARF-102-85-295 & Keep & ARF-102-85-295* ${ }^{\dagger}$ \\
\hline ARF-102-85-355 & Keep & ARF-102-85-355* ${ }^{\dagger}$ \\
\hline ARF-102-85-365 & Keep & ARF-102-85-365* ${ }^{\dagger}$ \\
\hline
\end{tabular}

- This group of containers is the subgroup of the Hanford "ARF" material, which includes chloride-bearing Rocky Flats ER scrape-out material that was sent to Hanford for recovery. These containers were identified as foundry oxide material, based on the PG peak area ratios for $\mathrm{Na}: \mathrm{Cl}$ and $\mathrm{Mg}: \mathrm{Na}(9.5 \leq \mathrm{Na}: \mathrm{Cl} \leq 15.5$ and $\mathrm{Mg}: \mathrm{Na} \leq 0.25) .^{10,14}$

- The assay values for this group range from 47.2 to $80.6 \mathrm{wt} \%$ actinide. ${ }^{8}$ The plutonium assay ranges from 47.1 to $80.6 \mathrm{wt} \%$. Uranium was found in one container at a concentration of $5.5 \mathrm{wt} \%$.

- PG analysis was completed for all 293 containers in this group. The results show that all 293 containers have a chloride salt composition similar to that of ER scrap. The chlorine concentration for the materials in this group range from 2.2 to $10.6 \mathrm{wt} \%$. Beryllium is present in 23 containers and ranges in concentration from 0.02 to $0.74 \mathrm{wt} \%$. None of the containers in this group are "potentially similar" or "most similar" to MIS item 011589A.

- This group is represented by the five Hanford ARF samples in the MIS program. MIS item 011589A does not represent any containers in this group, based on the results from PG analysis.

\section{Rocky Flats Miscellaneous Scrap Oxide at Hanford}

- 3013 Taxon: PyroOx-HN-RF-MiscOx

- Formerly: Subset of Hanford 1E

- Number of 3013 Containers: 122

- Representation in MIS:

\begin{tabular}{|c|c|c|}
\hline Original & Add/Remove/Keep & Current \\
\hline ARF-102-85-114-1 & Keep & ARF-102-85-114-1 ${ }^{*}$ \\
\hline ARF-102-85-223 & Keep & ARF-102-85-223* ${ }^{\dagger}$ \\
\hline ARF-102-85-295 & Keep & ARF-102-85-295* ${ }^{\dagger}$ \\
\hline ARF-102-85-355 & Keep & ARF-102-85-355 ${ }^{\star^{\dagger}}$ \\
\hline ARF-102-85-365 & Keep & ARF-102-85-365* ${ }^{\dagger}$ \\
\hline & Add & $011589 A^{\star^{\dagger \mp}}$ \\
\hline
\end{tabular}

- This group of containers is the subgroup of the Hanford “ARF” material, which includes Rocky Flats material at Hanford with and without chlorine. The portion 
with chlorine has a composition unlike that of both ER scrap and foundry oxide, based on the PG peak area ratios for $\mathrm{Na}: \mathrm{Cl}$ and $\mathrm{Mg}: \mathrm{Na}$. These materials may include burnt anode heel and other impure plutonium oxides.

- The assay values for this group range from 44.8 to $87.2 \mathrm{wt} \%$ actinide. ${ }^{8}$ The plutonium assay ranges from 44.7 to $87.1 \mathrm{wt} \%$ actinide. Uranium was found in one container at $14.5 \mathrm{wt} \%$.

- $\quad$ PG analysis was completed on all 122 containers. Chloride was detected in 49 containers and ranges in concentration from 0.3 to $8.5 \mathrm{wt} \%$. Fluorine (without chlorine) was found in 45 containers and ranges in concentration from 0.3 to 2.4 $w t \%$. Prompt gamma did not detect impurities in six containers, and the remaining 22 containers have "other impurities," including aluminum, beryllium, magnesium, phosphorus, and sodium. Beryllium was found in 46 containers and ranges from 0.02 to $1.7 \mathrm{wt} \%$. The chloride-bearing containers in this group do not match either the ER scrap or foundry oxide material, based on the $\mathrm{Na}: \mathrm{Cl}$ and Mg:Na peak area ratios. Eleven containers in this group are "potentially similar" to 011589A because they have a salt composition unlike that of ER scrap, and they have a moisture concentration of $0.1 \mathrm{wt} \%$ or higher.

- This group is represented by the five Hanford ARF samples in the MIS program in addition to MIS item 011589A, which represents the 11 containers that are "potentially similar" to it, based on PG and moisture. ${ }^{10}$

\section{Magnesium Hydroxide Precipitation at Hanford}

- 3013 Taxon: MgOHPPt-HN

- Formerly: Subset of Hanford 1F

- Number of 3013 Containers: 121

- Representation in MIS:

\begin{tabular}{|c|c|c|}
\hline Original & Add/Remove/Keep & Current \\
\hline $66-00-11-355$ & Keep & $66-00-11-355^{{ }^{\dagger}}$ \\
\hline $66-01-01-439$ & Keep & $66-01-01-43 *^{{ }^{\dagger}}$ \\
\hline ATL27960 & Remove & \\
\hline BLO-39-11-14-004 & Remove & \\
\hline PBO-47-09-012-023 & Remove & \\
\hline $\begin{array}{l}\text { *Small-Scale Surveillance } \\
\text { tFull Characterization, including chemistry, surface area, density, etc. }\end{array}$ \\
\hline
\end{tabular}

- This group contains impure and scrap plutonium oxides "recently produced" from magnesium hydroxide precipitation. ${ }^{3}$ The primary material type of the input items as recorded in the ISP database is “MGO," which indicates the magnesiumhydroxide precipitation process.

- The assay values for this group range from 30.4 to $68.3 \mathrm{wt} \%$ actinide. ${ }^{8}$ The plutonium assay ranges from 18.8 to $68.0 \mathrm{wt} \%$. Uranium is present in 21 containers and is found in concentrations up to $18.9 \mathrm{wt} \%$.

- PG analysis was completed for all 121 containers and shows that the magnesium hydroxide precipitate and the oxalate precipitate materials are distinguishable by the concentration of magnesium and sodium and the $\mathrm{Na}: \mathrm{Mg}$ ratio. The magnesium hydroxide precipitate materials typically have less than $1 \mathrm{wt} \%$ sodium, greater 
than $3 \mathrm{wt} \%$ magnesium, and may contain phosphorus. By Na:Mg ratio, the magnesium hydroxide precipitate materials have ratios of less than 0.1 . Chloride was not detected by PG analysis for any of the containers in this group. Fluorine was detected in 16 containers and ranges from 0.03 up to about $0.2 \mathrm{wt} \%$. The estimated magnesium concentrations range from less than the detection limit of 0.1 to $52 \mathrm{wt} \%$. Beryllium was not detected in any of the containers in this group.

- MIS items 66-00-11-355 and 66-01-01-439 were produced by the magnesium hydroxide precipitation process at Hanford and represent all 121 containers in this group.

- MIS items considered but not used for representation:

\begin{tabular}{|c|l|}
\hline MIS Item & \multicolumn{1}{c|}{ Reason not used } \\
\hline 39-01153A & $\begin{array}{l}\text { This MIS item has an assay value well below 30 } \\
\text { wt\% actinide, which was the minimum actinide } \\
\text { concentration allowed in a 3013 container. In } \\
\text { addition, this MIS item was not included in the } \\
\text { small-scale, shelf-life experiments. }\end{array}$ \\
\hline ATL27960 & $\begin{array}{l}\text { This MIS item was not well characterized by } \\
\text { process history or analytical chemistry, and material } \\
\text { is no longer available in the MIS inventory. }\end{array}$ \\
\hline BLO-39-11-14-004 & $\begin{array}{l}\text { This MIS item was produced through oxalate } \\
\text { precipitation. It lacks the major impurities found in } \\
\text { oxide produced through magnesium hydroxide } \\
\text { precipitation. The actinide concentration is also out } \\
\text { of the range for this group. }\end{array}$ \\
\hline PBO-47-09-012-023 & $\begin{array}{l}\text { This MIS item was produced through oxalate } \\
\text { precipitation. It lacks the major impurities found in } \\
\text { oxide produced through magnesium hydroxide } \\
\text { precipitation. The actinide concentration is also out } \\
\text { of the range for this group. }\end{array}$ \\
\hline
\end{tabular}

\section{Oxalate Precipitation of Impure Plutonium Nitrate Solutions at Hanford}

- 3013 Taxon: OxlPPt-HN-Impure

- Formerly: Subset of Hanford 1F

- Number of 3013 Containers: 54

- Representation in MIS

\begin{tabular}{|c|c|c|}
\hline Original & Add/Remove/Keep & Current \\
\hline $66-00-11-355$ & Keep & $66-00-11-355^{{ }^{\dagger}}$ \\
\hline $66-01-01-439$ & Keep & $66-01-01-43 *^{{ }^{\dagger}}$ \\
\hline ATL27960 & Remove & \\
\hline BLO-39-11-14-004 & Keep & BLO-39-11-14-004 ${ }^{\dagger}$ \\
\hline PBO-47-09-012-023 & Keep & PBO-47-09-012- \\
& & $02 *^{{ }^{\dagger}}$ \\
\hline *Small-Scale Surveillance \\
tFull Characterization, including chemistry, surface area, density, etc.
\end{tabular}


- This group contains impure and scrap plutonium oxides recently produced from oxalate precipitation. ${ }^{3}$ The primary material type of the input items as recorded in the ISP database is "OX," and the secondary material type is either " $\mathrm{F}$ " or "C\&D." This indicates that the plutonium oxide was produced from the oxalate precipitation process from filtrate and "cats and dogs."

- The assay values for this group range from 33.4 to $77.4 \mathrm{wt} \%$ actinide. ${ }^{8}$ The plutonium assay ranges from 33.3 to $77.2 \mathrm{wt} \%$. Uranium is present in one container at $4 \mathrm{wt} \%$.

- PG analysis has been completed for all 54 containers. Chloride was not detected in any containers. Fluorine (without chlorine) was found in 52 containers and ranges in concentration from $0.05 \mathrm{wt} \%$ to $0.75 \mathrm{wt} \%$. The remaining two containers have "other impurities," including aluminum, magnesium, phosphorus, and sodium. Beryllium was not detected in any of the containers in this group. The concentration of magnesium in the material in this group ranges from 0.2 to $38 \mathrm{wt} \%$, with only 11 containers greater than $3 \mathrm{wt} \%$ magnesium. The sodium concentration ranges from 0.3 to $12 \mathrm{wt} \%$. Fifty containers have a sodium concentration greater that $2.4 \mathrm{wt} \%$. The magnesium hydroxide precipitate and the oxalate precipitate materials are distinguishable by the concentration of magnesium and sodium and the Na:Mg ratio. The magnesium hydroxide precipitate materials typically have less than $1 \mathrm{wt} \%$ sodium and greater than 3 $\mathrm{wt} \%$ magnesium. By Na:Mg ratio, the magnesium hydroxide precipitate materials have ratios less than 0.1 .

- MIS items BLO-39-11-14-004 and PBO-47-09-012-023 represent this material, based on their process of origin; although, their actinide concentration is higher than the material in this group. MIS items 66-00-11-355 and 66-01-01-439 also represent these materials, based on the impurities present in the feed and the low actinide concentrations.

- MIS items considered but not used for representation:

\begin{tabular}{|c|l|}
\hline MIS Item & \multicolumn{1}{c|}{ Reason not used } \\
\hline 39-01153A & $\begin{array}{l}\text { This MIS item has an assay value well below 30 } \\
\text { wt\% actinide, which was the minimum actinide } \\
\text { concentration allowed in a 3013 container. In } \\
\text { addition, this MIS item was not included in the } \\
\text { small-scale, shelf-life experiments. }\end{array}$ \\
\hline ATL27960 & $\begin{array}{l}\text { This MIS item was not well characterized by } \\
\text { process history or analytical chemistry, and material } \\
\text { is no longer available in the MIS inventory. }\end{array}$ \\
\hline
\end{tabular}

\section{Oxalate Precipitation of Pure Mixed Uranium/Plutonium Nitrate Solutions at Hanford}

- 3013 Taxon: OxlPPt-HN-MixedPure

- Formerly: Hanford $1 \mathrm{G}$

- Number of 3013 Containers: 52 
- Representation in MIS

\begin{tabular}{|c|c|c|}
\hline Original & Add/Remove/Keep & Current \\
\hline BLO-39-11-14-004 & Keep & BLO-39-11-14-004* \\
\hline PBO-47-09-012-023 & Keep & PBO-47-09-012-023* ${ }^{\dagger}$ \\
\hline ATL27960 & Remove & \\
\hline 669194 & Keep & $669194^{*^{\dagger}}$ \\
\hline CAN92 & Keep & CAN92* ${ }^{\dagger}$ \\
\hline
\end{tabular}

- This group contains product quality mixed oxides recently produced from pure mixed uranium/plutonium nitrate feeds. ${ }^{3}$ The primary material type of the input items as recorded in the ISP database is "OX," and the secondary is material type is "CML." This indicates that the material was produced by oxalate precipitation of solutions from the critical mass laboratory.

- The assay values for this group range from $71.1 \mathrm{wt} \%$ to $90.1 \mathrm{wt} \%$ actinide. ${ }^{8}$ The plutonium assay ranges from 14.1 to $85.2 \mathrm{wt} \%$. Uranium is present in all 52 containers and is found in concentrations up to $60.9 \mathrm{wt} \%$.

- PG analysis was completed on all 52 containers. Chlorine and fluorine were not detected in any of the containers. Prompt gamma did not detect impurities in 13 containers, and the remaining containers have low concentrations (less than 1 $\mathrm{wt} \%$ ) of aluminum, magnesium, and sodium. Beryllium was not detected in any of the containers in this group.

- MIS items BLO-39-11-14-004 and PBO-47-09-012-023 represent this material, based on their process of origin; although their actinide concentration is higher than some of the materials in this group. It was decided that MIS items 669194 and CAN92 also represent this material because they are high-purity mixed oxides. 
- MIS items considered but not used for representation:

\begin{tabular}{|c|l|}
\hline MIS Item & \multicolumn{1}{c|}{ Reason not used } \\
\hline MISSTD-1 & $\begin{array}{l}\text { This MIS item was not stabilized at } 950^{\circ} \mathrm{C} \text { and has } \\
\text { a high surface area, which is not expected to be } \\
\text { representative of these materials. }\end{array}$ \\
\hline MISSTD-2 & $\begin{array}{l}\text { This MIS item was not characterized and was not } \\
\text { included in small-scale, shelf-life experiments. }\end{array}$ \\
\hline CXLNM1 & $\begin{array}{l}\text { This MIS item was not characterized and was not } \\
\text { included in small-scale, shelf-life experiments. In } \\
\text { addition, the material in this sample was } \\
\text { precipitated from chloride instead of nitrate. }\end{array}$ \\
\hline PEOR3258 & $\begin{array}{l}\text { This MIS item has limited characterization data and } \\
\text { was not included in small-scale, shelf-life } \\
\text { experiments. }\end{array}$ \\
\hline PEOF1 & $\begin{array}{l}\text { This MIS item, although produced through oxalate } \\
\text { precipitation, was produced at LANL and is not a } \\
\text { mixed oxide. }\end{array}$ \\
\hline ATL27960 & $\begin{array}{l}\text { This MIS item was not well characterized by } \\
\text { process history or analytical chemistry, and material } \\
\text { is no longer available in the MIS inventory. }\end{array}$ \\
\hline
\end{tabular}

\section{Impure and Scrap Plutonium Oxides 80-85 wt\% from Hanford PFP and 300 Area}

- 3013 Taxon: ScrapOx-HN-Hi

- Formerly: Hanford 2A

- Number of 3013 Containers: 32

- Representation in MIS

\begin{tabular}{|c|c|c|}
\hline Original & Add/Remove/Keep & Current \\
\hline BLO-39-11-14-004 & Keep & BLO-39-11-14-004* ${ }^{\dagger}$ \\
\hline PBO-47-09-012-023 & Keep & PBO-47-09-012-023* ${ }^{\dagger}$ \\
\hline 011608 & Keep & $011608^{\dagger}$ \\
\hline \multirow[t]{4}{*}{ PPSL-365 } & Keep & PPSL-365* ${ }^{\dagger}$ \\
\hline & Add & $\mathrm{TS}^{2} 07001^{*^{\dagger}}$ \\
\hline & Add & $07221730^{\dagger}$ \\
\hline & Add & ARF-102-85-114-1* ${ }^{\dagger}$ \\
\hline
\end{tabular}

- This group contains impure and scrap plutonium oxides from PFP and the 300 area. $^{3}$

- The assay values for this group range from 80.0 to $84.8 \mathrm{wt} \%$ actinide. ${ }^{8}$ The plutonium assay ranges from 75.8 to $84.5 \mathrm{wt} \%$. Uranium is present in one container at $4.7 \mathrm{wt} \%$.

- PG analysis was completed for all 32 containers. Chlorine was found in two containers at concentrations of 1.3 and $3.0 \mathrm{wt} \%$. Fluorine (without chlorine) was 
found in 12 containers and ranges in concentration from 0.02 to $3.0 \mathrm{wt} \%$; however, only one container has fluorine above $0.8 \mathrm{wt} \%$. Prompt gamma did not detect impurities in three containers, and the remaining 15 containers have "other impurities," including aluminum, beryllium, magnesium, phosphorus, and sodium. Beryllium was detected in two containers at $0.03 \mathrm{wt} \%$.

- These materials are represented by MIS items from oxalate precipitation (PBO47-09-012-023 and BLO-39-11-14-004), direct denitration (PPSL-365), and metal oxidation (011608). Additional metal oxidation MIS items from RFETS and Hanford (TS707001, 07221730 and ARF-102-85-114-1) were added, based on process similarity. MIS item TS707001 also provides a match for the materials with detectable chloride.

- MIS items considered but not used for representation:

\begin{tabular}{|c|c|}
\hline MIS Item & Reason not used \\
\hline 011589A & $\begin{array}{l}\text { The chloride-bearing containers in this group were } \\
\text { not found to be "similar" in salt composition or } \\
\text { moisture to this sample. }\end{array}$ \\
\hline 101707001 & $\begin{array}{l}\text { This MIS item contains less than } 30 \mathrm{wt} \% \text { actinide. } \\
\text { This sample was also not characterized in the MIS } \\
\text { program and is not included in small-scale, shelf-life } \\
\text { experiments. }\end{array}$ \\
\hline MT1490 & $\begin{array}{l}\text { This MIS item originated in R\&D operations at } \\
\text { RFETS and has a considerable amount of } \\
\text { neptunium present. }\end{array}$ \\
\hline TS707013 & $\begin{array}{l}\text { This MIS item is high in chloride and outside of the } \\
\text { range of actinide concentration. }\end{array}$ \\
\hline MISSTD-1 & $\begin{array}{l}\text { This MIS item was not stabilized at } 950^{\circ} \mathrm{C} \text { and has } \\
\text { a high surface area, which is not expected to be } \\
\text { representative of these materials. }\end{array}$ \\
\hline MISSTD-2 & $\begin{array}{l}\text { This MIS item was not characterized and was not } \\
\text { included in small-scale, shelf-life experiments. }\end{array}$ \\
\hline CXLNM1 & $\begin{array}{l}\text { This MIS item was not characterized and was not } \\
\text { included in small-scale, shelf-life experiments. In } \\
\text { addition, the material in this sample was } \\
\text { precipitated from chloride instead of nitrate. }\end{array}$ \\
\hline PEOR3258 & $\begin{array}{l}\text { This MIS item has limited characterization data and } \\
\text { was not included in small-scale, shelf-life } \\
\text { experiments. }\end{array}$ \\
\hline
\end{tabular}

\section{Impure and Scrap Oxides 30-80 wt\% from Aqueous Recovery, Oxalate Precipitation, and Hydrofluorination from Hanford}

- 3013 Taxon: ScrapOx-HN-Lo

- Formerly: Hanford 2B

- Number of 3013 Containers: 173 
- Representation in MIS

\begin{tabular}{|c|c|c|}
\hline Original & Add/Remove/Keep & Current \\
\hline 07032282A & Keep & $07032282 A^{*^{\dagger}}$ \\
\hline 07242201A & Keep & $07242201 A^{*^{\dagger}}$ \\
\hline 07242165A & Keep & $07242165 A^{*^{\dagger}}$ \\
\hline ATL27960 & Remove & \\
\hline PPSL-365 & Keep & PPSL-365* ${ }^{\dagger}$ \\
\hline C06032A & Remove & \\
\hline $07242141 \mathrm{~A}$ & Remove & \\
\hline & Add & $41-85-08-1379^{*^{\dagger}}$ \\
\hline & Add & $63-88-06-121^{*^{\dagger}}$ \\
\hline & Add & $64-85-12-1858^{*^{\dagger}}$ \\
\hline
\end{tabular}

- The materials in this group are low purity (less that $80 \%$ plutonium) scrap oxides that originated in PFP from aqueous recovery, oxalate precipitation, and hydrofluorination. ${ }^{3}$

- The assay values for this group range from 34.2 to $80.5 \mathrm{wt} \%$ actinide. ${ }^{8}$ The plutonium assay ranges from 25.0 to $79.3 \mathrm{wt} \%$. Uranium is present in 25 containers and is found in concentrations up to $25.6 \mathrm{wt} \%$.

- PG analysis was completed for all 173 containers in this group. Chlorine was found in 28 containers and ranges in concentration from 0.3 to $7.5 \mathrm{wt} \%$. Fluorine (without chloride) was found in 120 containers and ranges from 0.01 to $7.7 \mathrm{wt} \%$. Most of the containers with fluoride also have aluminum and magnesium as impurities. Prompt gamma did not detect impurities in five containers, and the remaining 20 containers have "other impurities" including aluminum, beryllium, phosphorus, magnesium, sodium. Beryllium was detected in 31 containers and ranges in concentration from 0.02 to $0.3 \mathrm{wt} \%$.

- These materials are primarily represented by scrap oxide samples (41-85-08-1379, 63-88-06-121, 64-85-12-1858) from PFP. These MIS items were included in the MIS program after the original represented document for Hanford was completed. In addition, several RFETS MIS items from dissolution residuals (07032282A, 07242201A, and 07242165A) were selected for representation along with Hanford scrap oxides and one MIS item from direct denitration (PPSL-365). These are appropriate, based on process similarity and impurities. PPSL-365 (direct denitration) is appropriate because it represents oxides produced by calcining nitrate. 
- MIS items considered but not used for representation:

\begin{tabular}{|c|c|}
\hline MIS Item & Reason not used \\
\hline ATL27960 & $\begin{array}{l}\text { This MIS item was not well characterized by } \\
\text { process history or analytical chemistry, and material } \\
\text { is no longer available in the MIS inventory. }\end{array}$ \\
\hline C06032A & $\begin{array}{l}\text { This MIS item was removed from the list because } \\
\text { Rocky Flats oxide screenings material does not } \\
\text { represent this group of material. }\end{array}$ \\
\hline $07242141 \mathrm{~A}$ & $\begin{array}{l}\text { This MIS item was removed from the list because } \\
\text { Rocky Flats oxide screenings material does not } \\
\text { represent this group of material. }\end{array}$ \\
\hline 07242326A & $\begin{array}{l}\text { This MIS item contains less than } 30 \text { wt } \% \text { actinide. } \\
\text { This sample was also not characterized in the MIS } \\
\text { program and is not included in small-scale, shelf-life } \\
\text { experiments. }\end{array}$ \\
\hline 07242243A & $\begin{array}{l}\text { This MIS item contains less than } 30 \mathrm{wt} \% \text { actinide. } \\
\text { This sample was also not characterized in the MIS } \\
\text { program and is not included in small-scale, shelf-life } \\
\text { experiments. }\end{array}$ \\
\hline 62750 & $\begin{array}{l}\text { The assay value for this MIS item is outside of the } \\
\text { range for this group. }\end{array}$ \\
\hline
\end{tabular}

\section{Oxide from PFP NDA Sources at Hanford}

- 3013 Taxon: OxlPPt-HN-Sources

- Formerly: Hanford 2C

- Number of 3013 Containers: 7

- Representation in MIS

\begin{tabular}{|c|c|c|}
\hline Original & Add/Remove/Keep & Current \\
\hline BLO-39-11-14-004 & Keep & BLO-39-11-14-004* \\
\hline PBO-47-09-012-023 & Keep & PBO-47-09-012-023* \\
\hline PSU-84-06-05 & Remove & \\
\hline & Add & PEOF1*1 \\
\hline
\end{tabular}

- The materials in this group are impure and scrap plutonium oxides from PFP fabricated NDA sources. ${ }^{3}$ The material is in an inert matrix, and most of the oxide was produced from oxalate precipitation.

- The assay values for this group range from 72.4 to $87.3 \mathrm{wt} \%$ actinide. ${ }^{8}$ The plutonium assay ranges from 72.1 to $86.8 \mathrm{wt} \%$. None of the containers have uranium.

- PG analysis was completed for all seven containers, and the results do not indicate chloride or fluoride in any containers. Prompt gamma did not detect impurities in one container, and the remaining six containers had "other impurities," including aluminum, magnesium, and sodium. Beryllium was not detected in any of the containers in this group. 
- These materials are represented by BLO-39-11-14-004, PBO-47-09-012-023, and PEOF1, based on the assay and process history of these samples.

- MIS items considered but not used for representation:

\begin{tabular}{|c|l|}
\hline MIS Item & \multicolumn{1}{c|}{ Reason not used } \\
\hline MISSTD-1 & $\begin{array}{l}\text { This MIS item was not stabilized at } 950^{\circ} \mathrm{C} \text { and has } \\
\text { a high surface area, which is not expected to be } \\
\text { representative of these materials. }\end{array}$ \\
\hline MISSTD-2 & $\begin{array}{l}\text { This MIS item was not characterized and was not } \\
\text { included in small-scale, shelf-life experiments. }\end{array}$ \\
\hline CXLNM1 & $\begin{array}{l}\text { This MIS item was not characterized and was not } \\
\text { included in small-scale, shelf-life experiments. In } \\
\text { addition, the material in this sample was } \\
\text { precipitated from chloride instead of nitrate. }\end{array}$ \\
\hline PEOR3258 & $\begin{array}{l}\text { This MIS item has limited characterization data and } \\
\text { was not included in small-scale, shelf-life } \\
\text { experiments. }\end{array}$ \\
\hline PSU-84-06-05 & $\begin{array}{l}\text { This MIS item was not produced in the same } \\
\text { process. In addition, this material contains uranium, } \\
\text { which is not present in the materials in this group. }\end{array}$ \\
\hline
\end{tabular}

\section{High Purity Mixed Oxides at Hanford}

- 3013 Taxon: PuUOx-HN-Misc

- Formerly: Hanford 2D

- Number of 3013 Containers: 231

- Representation in MIS

\begin{tabular}{|c|c|c|}
\hline Original & Add/Remove/Keep & Current \\
\hline SCP711-46 & Keep & SCP711-46 $^{\dagger}$ \\
\hline SCP711-56 & Keep & SCP711-56 $^{{ }^{\dagger}}$ \\
\hline MOXSCP-COM & Remove & \\
\hline 669194 & Keep & $669194^{*^{\dagger}}$ \\
\hline CAN92 & Keep & CAN92 $^{{ }^{\dagger}}$ \\
\hline 5501407 & Keep & $5501407^{{ }^{\dagger}}$ \\
\hline \multicolumn{2}{|c|}{ Add } & CXL1685 $^{\dagger}$ \\
\hline $\begin{array}{l}\text { *Small-Scale Surveillance } \\
\text { tFull Characterization, including chemistry, surface area, density, etc. }\end{array}$
\end{tabular}

- The material in this group originated in oxalate precipitation process and included pure and impure mixed oxides. ${ }^{3}$

- $\quad$ The assay values for this group range from 59.3 to $96.6 \mathrm{wt} \%$ actinide. ${ }^{8}$ The plutonium assay ranges from 0.7 to $79.6 \mathrm{wt} \%$. Uranium is present in all of the containers and ranges in concentration from 2.5 to $90 \mathrm{wt} \%$.

- $\quad$ PG analysis was completed for all 231 containers. Chlorine was detected in 3 containers at concentrations of $0.2,1.5$, and $8.4 \mathrm{wt} \%$. Fluorine (without chlorine) was found in five containers and ranges in concentration from 0.02 to $4.6 \mathrm{wt} \%$. Prompt gamma did not detect impurities in 125 containers, and the remaining 98 
containers have "other impurities," including aluminum, magnesium, and sodium. Beryllium was not detected in any of the containers in this group.

- The materials in this group are represented by mixed plutonium/uranium oxides selected from the LANL and RFETS MIS items. MIS item CXL1685 was added to the list because it is plutonium oxalate mixed with depleted uranium.

- MIS items considered but not used for representation:

\begin{tabular}{|c|l|}
\hline MIS Item & \multicolumn{1}{c|}{ Reason not used } \\
\hline MOXSCP-COM & $\begin{array}{l}\text { This MIS item, although similar in actinide content, } \\
\text { was not included because it was not characterized } \\
\text { and not included in small-scale, shelf-life } \\
\text { experiments. }\end{array}$ \\
\hline PuUOXBC05 & $\begin{array}{l}\text { This MIS item, although a mixed oxide, is below the } \\
\text { assay range for this group. In addition, this sample } \\
\text { is not completely characterized, and its process } \\
\text { history is unknown. Further, this sample was not } \\
\text { included in small-scale, shelf-life experiments. }\end{array}$ \\
\hline 1685 & $\begin{array}{l}\text { This MIS item is depleted uranium oxide that does } \\
\text { not contain plutonium. In addition, this sample was } \\
\text { not characterized and not included in small-scale, } \\
\text { shelf-life experiments. }\end{array}$ \\
\hline BMU & $\begin{array}{l}\text { This MIS item is enriched uranium oxide that does } \\
\text { not contain plutonium. In addition, this sample was } \\
\text { not characterized and not included in small-scale, } \\
\text { shelf-life experiments. }\end{array}$ \\
\hline
\end{tabular}

\section{Mixed Oxides from Alloy Oxidation at Hanford}

- 3013 Taxon: PuUOx-HN-Alloy

- Formerly: Hanford 2E

- Number of 3013 Containers: 12

- Representation in MIS

\begin{tabular}{|c|c|c|}
\hline Original & Add/Remove/Keep & Current \\
\hline 5501407 & Keep & $5501407 *^{\dagger}$ \\
\hline 011589A & Remove & \\
\hline C06032A & Remove & \\
\hline 07242141A & Keep & $07242141 A^{*^{\dagger}}$ \\
\hline & Add & $669194^{*^{\dagger}}$ \\
\hline
\end{tabular}

- The materials in this group consist of screenings removed from impure and mixed oxides originating in the metal oxidation process. ${ }^{3}$

- The assay values for this group range from 39.0 to $78.5 \mathrm{wt} \%$ actinide. ${ }^{8}$ The plutonium assay ranges from 9.5 to $28.4 \mathrm{wt} \%$. Uranium is present in all 12 containers and is found in concentrations ranging from 25.4 to $55.4 \mathrm{wt} \%$.

- PG analysis was completed for all 12 containers. Prompt gamma did not detect chlorine. Fluorine was found in one container at $0.2 \mathrm{wt} \%$. Prompt gamma did not 
detect impurities in two containers, and the remaining nine containers have "other impurities,” including aluminum, magnesium, and sodium. Beryllium was not detected in any containers.

- The MIS items that represent this group were chosen from mixed Rocky Flats plutonium/uranium oxides (5501407 and 669194) and oxide screenings from foundry operations at Rocky Flats (07242141A). MIS item C06032A is also considered oxide screenings material from Rocky Flats, but high chloride content of C06032A shows that this MIS item may have originated in one of the Rocky Flats molten salt purification processes. However, the impurities found in 07242141A show that those oxide screenings likely originated in the foundry and are therefore representative of this material.

- MIS items considered but not used for representation:

\begin{tabular}{|l|l|}
\hline MIS Item & \multicolumn{1}{c|}{ Reason not used } \\
\hline 011589A & $\begin{array}{l}\text { Chloride is present in 011589A, but no chloride is } \\
\text { detected in the 3013 containers by PG analysis. In } \\
\text { addition, this MIS item exhibited unique behavior in } \\
\text { small-scale, shelf-life experiments that is not } \\
\text { considered likely in these materials. }\end{array}$ \\
\hline C06032A & $\begin{array}{l}\text { The high-chloride content of this MIS item clearly } \\
\text { shows that this MIS item has a different origin and } \\
\text { is therefore not representative of the materials in } \\
\text { this group. }\end{array}$ \\
\hline
\end{tabular}

\section{Peroxide Precipitation/Calcination at Rocky Flats}

- 3013 Taxon: PeroxPPT-RF

- Formerly: Rocky Flats 1A

- Number of 3013 Containers: 44

- Representation in MIS

\begin{tabular}{|c|c|c|}
\hline Original & Add/Remove/Keep & Current \\
\hline 1000089 & Keep & $1000089^{*^{\dagger}}$ \\
\hline 07161856 & Keep & $07161856^{*^{\dagger}}$ \\
\hline \multicolumn{2}{|c|}{} \\
*Small-Scale Surveillance \\
tFull Characterization, including chemistry, surface area, density, etc.
\end{tabular}

- The materials in this process were produced by peroxide precipitation from nitrate solution at Rocky Flats. ${ }^{4}$

- The assay values for this group range from 65.1 to $91.0 \mathrm{wt} \%$ actinide. ${ }^{8}$ The plutonium assay values range from 47.0 to $88.4 \mathrm{wt} \%$. Uranium is present in one container at a concentration of $43.8 \mathrm{wt} \%$.

- PG analysis was completed for 43 containers. Chlorine was found in two containers at concentrations around $1.0 \mathrm{wt} \%$. Fluorine (without chlorine) was found in 22 containers and ranges in concentration from 0.2 to $3.4 \mathrm{wt} \%$. Prompt gamma did not detect impurities in ten containers, and the remaining nine containers have "other impurities," including beryllium, magnesium, and sodium. Beryllium was detected in one container at about $0.3 \mathrm{wt} \%$. 
- The materials in this group are represented by MIS items from the peroxide precipitation process. The representation for this group remains unchanged.

- MIS items considered but not used for representation: none.

\section{Process Oxides from Metal Oxidation at Rocky Flats}

- 3013 Taxon: MetalOx-RF

- Formerly: Rocky Flats 1B \& 1C

- Number of 3013 Containers: 403

- Representation in MIS

\begin{tabular}{|c|c|c|}
\hline Original & Add/Remove/Keep & Current \\
\hline TS707001 & Keep & $\mathrm{TS} 707001^{*^{\dagger}}$ \\
\hline 011608 & Keep & $011608^{\dagger}$ \\
\hline 011589A & Keep & $011589 A^{* \ddagger}$ \\
\hline \multirow[t]{4}{*}{ MT1490 } & Keep & MT1490* \\
\hline & Add & $\mathrm{TS}^{2} 07013^{*^{\dagger}}$ \\
\hline & Add & $07221730^{\dagger}$ \\
\hline & Add & ARF-102-85-114-1 ${ }^{*^{\dagger}}$ \\
\hline
\end{tabular}

- The material in this group originated as plutonium metal that was calcined in air to oxidize the metal to oxide for aqueous recovery. ${ }^{4}$ This group also includes oxide that was brushed from metal. Also added to this group was the mixed plutonium-neptunium oxide produced from the oxidation of plutonium-neptunium alloy, because the presence of neptunium will not change the behavior of this material.

- The assay values show that the materials in this group are greater than $44.9 \mathrm{wt} \%$ actinide. ${ }^{8}$ The plutonium assay ranges from 41.5 to $87.8 \mathrm{wt} \%$. Uranium is present in 33 containers and is found in concentrations of up to $45.7 \mathrm{wt} \%$. The neptunium ranges in concentration from 0.01 to $3.33 \mathrm{wt} \%$ and is present in materials from both $1 \mathrm{~B}$ and $1 \mathrm{C}$.

- $\quad$ PG analysis was completed for 391 containers. Chlorine was found in 27 containers and ranges in concentration from 0.25 to $6.6 \mathrm{wt} \%$. Fluorine (without chlorine) was found in 140 containers and ranges in concentration from 0.05 to $2.4 \mathrm{wt} \%$. Prompt gamma did not detect impurities in 94 containers, and the remaining 130 containers have "other impurities," including aluminum, beryllium, magnesium, and sodium. Beryllium was found in 11 containers and ranges from 0.002 to $0.5 \mathrm{wt} \%$.

- The original MIS items representing this group are from the metal oxidation process and provide a good match in both impurities and actinide concentration. MIS items TS707013 and 07221730 were added, based on their process history and assay values. TS707013 represents the containers that have high chloride concentrations. MIS item 07221730 was added because of process similarity and assay. ARF-102-85-114-1 was a Hanford MIS item consisting of Rocky Flats material believed to be from metal oxidation. This MIS item is representative of 
the high assay containers with beryllium. MIS item 011589A represents two containers that are believed to be "most similar" to 011589A and 12 containers that are believed to be "potentially similar" to $011589 \mathrm{~A} .{ }^{10}$ (The $13^{\text {th }}$ potentially similar container R611398 is represented by PG).

- MIS items considered but not used for representation:

\begin{tabular}{|c|l|}
\hline MIS Item & \multicolumn{1}{c|}{ Reason not used } \\
\hline 101707001 & $\begin{array}{l}\text { This MIS item contains less than } 30 \mathrm{wt} \% \text { actinide. } \\
\text { This sample was also not characterized in the MIS } \\
\text { program and is not included in small-scale, shelf-life } \\
\text { experiments. }\end{array}$ \\
\hline
\end{tabular}

\section{Byproduct Plutonium/Uranium Mixed Oxides at Rocky Flats}

- 3013 Taxon: PuUOx-RF

- Formerly: Rocky Flats 2A

- Number of 3013 Containers: 279

- Representation in MIS

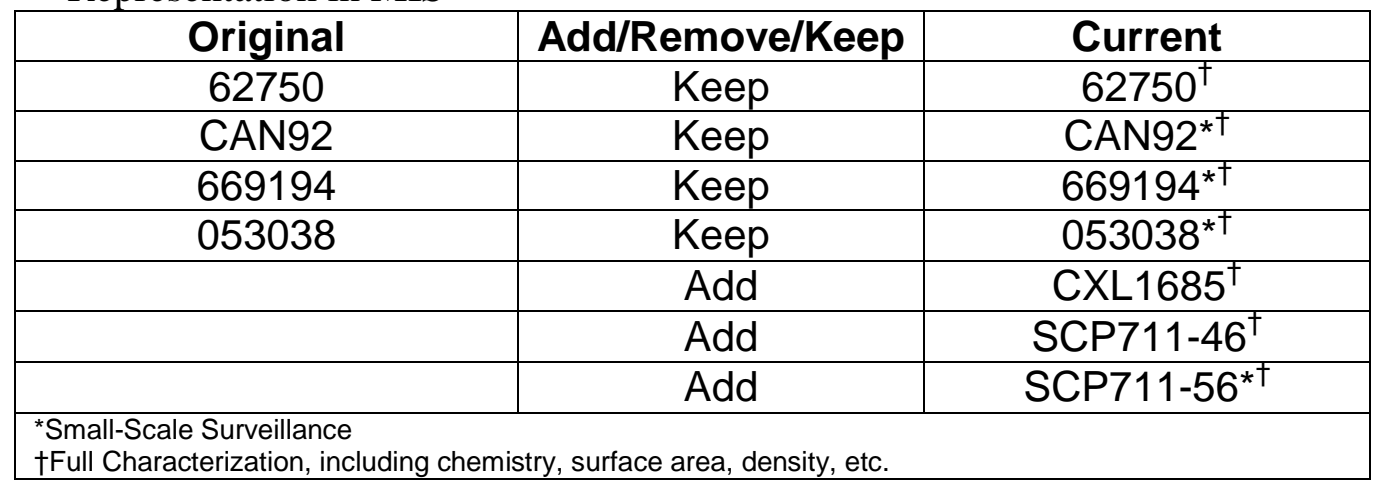

- The materials in this group are mostly Rocky Flats plutonium/uranium mixed oxides considered to be byproduct material, based on various information including the item description code (IDC), material balance area (MBA), and item name. $^{4}$

- The assay values show that the materials in this group are greater than $43.2 \mathrm{wt} \%$ actinide. ${ }^{8}$ The plutonium assay ranges from 3.7 to $86.6 \mathrm{wt} \%$. Uranium is present in 257 containers and is found in concentrations up to $94.0 \mathrm{wt} \%$.

- PG analysis was completed for 255 containers. Chlorine was found in three containers and ranges in concentration from 1.2 to $2.6 \mathrm{wt} \%$. Fluorine (without chlorine) was found in six containers and ranges in concentration from 0.04 to 2.4 wt\%. Prompt gamma did not detect impurities in 148 containers, and the remaining 98 containers have "other impurities" including aluminum, beryllium, magnesium, and sodium. Beryllium was found in 47 containers and ranges in concentration from 0.01 to $1.6 \mathrm{wt} \%$.

- This group was originally represented by four mixed-oxide MIS items in the MIS inventory from Rocky Flats, and the impurities sensitive to PG analysis are covered by these MIS items. In addition, three mixed-oxide MIS items from the MIS inventory were added to this group (CXL1685, SCP711-46, and SCP711- 
56). These MIS items have low-plutonium and high-uranium content and better represent the containers in this group that are mostly uranium oxide.

- MIS items considered but not used for representation:

\begin{tabular}{|c|c|}
\hline MIS Item & Reason not used \\
\hline MOXSCP-COM & $\begin{array}{l}\text { This MIS item, although similar in actinide content, } \\
\text { was not included because it was not characterized } \\
\text { and not included in small-scale, shelf-life } \\
\text { experiments. }\end{array}$ \\
\hline $\begin{array}{l}\text { CXL-BMU Mixtures } \\
\text { (1685, BMU, } \\
\text { BMUCXL70-30, } \\
\text { BMUCXL93-7, } \\
\text { CXL1685, } \\
\text { CXLBMU70-30, and } \\
\text { CXLBMU93-7) }\end{array}$ & $\begin{array}{l}\text { These MIS items were used for neutron moderation } \\
\text { and moisture experiments and not intended for } \\
\text { representation. Therefore, they were not } \\
\text { characterized and not included in small-scale, shelf- } \\
\text { life experiments. }\end{array}$ \\
\hline PSU-84-06-05 & $\begin{array}{l}\text { The material in this sample is considered specific to } \\
\text { pyrolytic processing of polycubes and is not } \\
\text { considered representative of this material. }\end{array}$ \\
\hline MISNE2 & $\begin{array}{l}\text { This MIS item is a mixture of Rocky Flats items } \\
\text { from various processes, which may have introduced } \\
\text { impurities that are not representative of this } \\
\text { process. In addition, gypsum and borax were added } \\
\text { to this material for neutron moderation experiments. }\end{array}$ \\
\hline PuUOXBC05 & $\begin{array}{l}\text { This MIS item, although a mixed oxide, is not } \\
\text { completely characterized, and its process history is } \\
\text { unknown. This sample was also not included in } \\
\text { small-scale, shelf-life experiments. }\end{array}$ \\
\hline
\end{tabular}

\section{Oxide from Pyrochemical Processes at Rocky Flats}

- 3013 Taxon: PyroOx-RF

- Formerly: Rocky Flats 2B

- Number of 3013 Containers: 160

- Representation in MIS

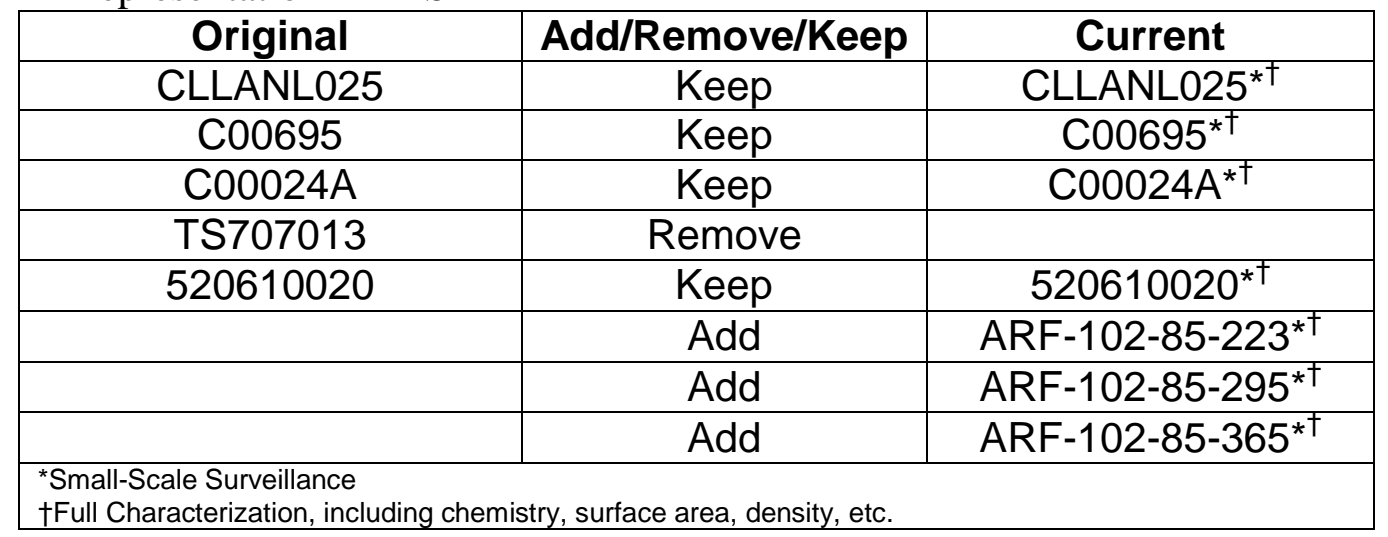


- This group of materials originated in pyrochemical processes. ${ }^{4}$ Most of the materials contain chloride salt impurities and came from the oxidation of scrapeout residuals from electrorefining.

- The assay values show that the materials in this group are greater than $43.6 \mathrm{wt} \%$ actinide. $^{8}$ The plutonium assay ranges from 13.1 to $91.8 \mathrm{wt} \%$. Uranium is present in 29 containers and is found in concentrations up to $52.5 \mathrm{wt} \%$.

- $\quad$ PG analysis was completed for 156 containers. Chlorine was found in 112 containers and ranges from 1.0 to $8.4 \mathrm{wt} \%$. Fluorine (without chlorine) was found in 13 containers and ranges in concentration from 0.07 to $2.8 \mathrm{wt} \%$. Prompt gamma did not detect impurities in 12 containers, and the remaining containers have "other impurities," including aluminum, beryllium, magnesium, and sodium. Beryllium was detected in nine containers and ranges from 0.08 to $1.0 \mathrm{wt} \%$.

- These containers were originally represented by four pyrochemical MIS items in the MIS inventory from Rocky Flats and one metal oxidation MIS item TS707013. Although it has a high chloride content, TS707013 was removed from the list because it originated in a metal oxidation process. Three additional samples from the MIS inventory were added to the MIS items that represent this group. These are Hanford samples consisting of Rocky Flats material believed to be from the same pyrochemical processes because of the high chloride content. Although other items in the MIS inventory have high chloride concentrations (053038, PMAXBS, etc.), it was decided that they do not represent this group because they are not specifically from Rocky Flats pyrochemical processing.

- MIS items considered but not used for representation:

\begin{tabular}{|c|l|}
\hline MIS Item & \multicolumn{1}{c|}{ Reason not used } \\
\hline 053038 & $\begin{array}{l}\text { Based on the chemical characterization, this } \\
\text { material has impurities that are unlike those found } \\
\text { in other MIS items in this group. }\end{array}$ \\
\hline PMAXBS & $\begin{array}{l}\text { This is a LANL-generated material produced by } \\
\text { mixing anode heel with ER salt, and may have } \\
\text { different impurities from those expected for this } \\
\text { group. }\end{array}$ \\
\hline ATL27960 & $\begin{array}{l}\text { This MIS item was not well characterized by } \\
\text { process history or analytical chemistry, and material } \\
\text { is no longer available in the MIS inventory. }\end{array}$ \\
\hline TS707013 & $\begin{array}{l}\text { This material originated in the metal oxidation } \\
\text { process. }\end{array}$ \\
\hline
\end{tabular}

\section{Oxide Residue from Dissolution at Rocky Flats}

- 3013 Taxon: DisResd-RF

- Formerly: Rocky Flats 2C

- Number of 3013 Containers: 37 
- Representation in MIS

\begin{tabular}{|c|c|c|}
\hline Original & Add/Remove/Keep & Current \\
\hline $07032282 \mathrm{~A}$ & Keep & $07032282 \mathrm{~A}^{*^{\dagger}}$ \\
\hline $07242165 \mathrm{~A}$ & Keep & $07242165 \mathrm{~A}^{*^{\dagger}}$ \\
\hline 07242201A & Keep & $07242201 \mathrm{~A}^{\star^{\dagger}}$ \\
\hline $\begin{array}{l}\text { *Small-Scale Surveillance } \\
\text { Fuull Characterization, including chemistry, surface area, density, etc. }\end{array}$
\end{tabular}

- These materials consist of the insoluble materials (high-fired oxide, carbon, and tantalum oxides) that remained after the dissolution process. ${ }^{4}$

- The assay values for this group range from 56.0 to $84.6 \mathrm{wt} \%$ actinide. ${ }^{8}$ The plutonium assay ranges from 46.0 to $84.4 \mathrm{wt} \%$. Uranium is present in 29 containers and is found in concentrations of up to $36.3 \mathrm{wt} \%$.

- PG analysis was completed for all 37 containers. Chlorine was found in one container at $0.7 \mathrm{wt} \%$. Fluorine (without chlorine) was found in 33 containers and ranges in concentration from 0.08 to $1.4 \mathrm{wt} \%$. Prompt gamma did not detect impurities in one container, and the remaining two containers have "other impurities," including aluminum, magnesium, and sodium. Beryllium was found in one container and ranges from $0.02 \mathrm{wt} \%$.

- This group is represented by three residue samples from oxide dissolution at Rocky Flats. The chloride and fluoride impurities are covered by the MIS items in this group. No changes to representation were made.

- MIS items considered but not used for representation:

\begin{tabular}{|c|l|}
\hline MIS Item & \multicolumn{1}{|c|}{ Reason not used } \\
\hline $07242326 \mathrm{~A}$ & $\begin{array}{l}\text { This MIS item contains less than 30 wt\% actinide. } \\
\text { This sample was also not characterized in the MIS } \\
\text { program and is not included in small-scale, shelf-life } \\
\text { experiments. }\end{array}$ \\
\hline 07242243A & $\begin{array}{l}\text { This MIS item contains less than 30 wt\% actinide. } \\
\text { This sample was also not characterized in the MIS } \\
\text { program and is not included in small-scale, shelf-life } \\
\text { experiments. }\end{array}$ \\
\hline 62750 & $\begin{array}{l}\text { Process history documentation indicates that this } \\
\text { material is a dissolution residue. However, this MIS } \\
\text { item has an actinide assay of } 86.4 \text { wt\%, which is } \\
\text { outside of the actinide assay range for the 3013 } \\
\text { containers in this group. }\end{array}$ \\
\hline
\end{tabular}

\section{Hydride Oxide from Rocky Flats}

- 3013 Taxon: HydrOx-RF

- Formerly: Rocky Flats 1D \& 2D

- Number of 3013 Containers: 86 (31 1D \& 55 2D) 
- Representation in MIS

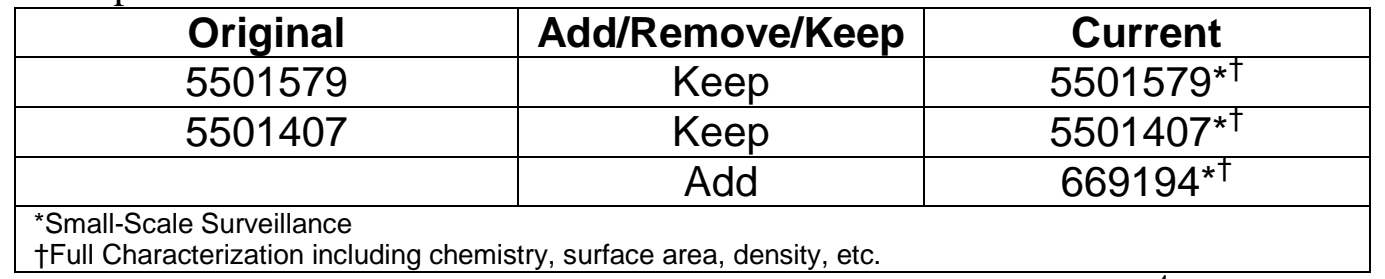

- This group of materials consists of oxide from hydride oxidation. ${ }^{4}$ This process was used to separate plutonium and uranium metals. The oxides in this group range from low plutonium/high uranium to high-plutonium/low-uranium oxides. The hydride material was originally split, based on whether the material was listed in the inventory as a product or a byproduct. The assay values from both groups show a large overlap; therefore, the groups were combined.

- The assay values show that the materials in this group are greater than $51.3 \mathrm{wt} \%$ actinide. $^{.}$The plutonium assay ranges from 4.58 to $86.8 \mathrm{wt} \%$. Uranium is present in 57 containers and is found in concentrations of up to $82.5 \mathrm{wt} \%$.

- PG analysis was completed for 83 containers. Chlorine was found in two containers at concentrations of 0.4 and $1.6 \mathrm{wt} \%$. Fluorine (without chlorine) was found in six containers ranges in concentration from 0.02 to $0.6 \mathrm{wt} \%$. Prompt gamma did not detect impurities in 53 containers, and the remaining 22 containers have "other impurities," including aluminum, beryllium, magnesium, and sodium. Beryllium was found in ten containers and ranges from 0.01 to $1.2 \mathrm{wt} \%$.

- The product and byproduct hydride oxide groups were combined, and the original MIS items were kept for representation. In addition, Rocky Flats sample 669194 was added, based on its process history, which states that it originated in special assembly projects. Materials from special assembly were generally sent for hydriding, and it is likely that this material is also a hydride oxide. In addition, it represents the material with higher concentrations of uranium oxide present.

- MIS items considered but not used for representation: None

\section{Screenings from Plutonium Oxidation at Rocky Flats}

- 3013 Taxon: OxScrns-RF

- Formerly: Rocky Flats 2E

- Number of 3013 Containers: 63

- Representation in MIS

\begin{tabular}{|c|c|c|}
\hline Original & Add/Remove/Keep & Current \\
\hline 07242141A & Keep & $07242141 A^{{ }^{\dagger}}$ \\
\hline C06032A & Keep & C06032A $*^{\dagger}$ \\
\hline $\begin{array}{l}\text { *Small-Scale Surveillance } \\
\text { tFull Characterization, including chemistry, surface area, density, etc. }\end{array}$ \\
\hline
\end{tabular}

- The material in this group consists of solid material chunks that were screened out of oxide powder. ${ }^{4}$ The material has multiple origins that include (1) glovebox floor sweepings, (2) oxide screenings following metal-to-oxide conversion at temperatures above $500^{\circ} \mathrm{C}$, and (3) screenings from chloride-bearing oxides that were being packaged for shipment to Hanford in the 1980s for recovery. 
- The assay values for this group range from 34.0 to greater than $70.3 \mathrm{wt} \%$ actinide. $^{8}$ The plutonium assay ranges from 34.0 to $70.2 \mathrm{wt} \%$. Uranium is present in 11 containers and is found in concentrations of up to $16.2 \mathrm{wt} \%$.

- PG analysis was completed for all 63 containers. Chlorine was detected in all 63 containers and ranges in concentration from 2.0 to $7.3 \mathrm{wt} \%$. Fluoride and beryllium are also present in a significant number of the chlorides with 19 containers having fluoride and 24 having beryllium present. The fluorine concentration ranges from 0.1 to $1.1 \mathrm{wt} \%$, and the beryllium concentration ranges from 0.03 to $0.4 \mathrm{wt} \%$.

- These materials are represented by two Rocky Flats oxide screenings samples: MIS items 07242141A and C06032A. MIS item 07242141A likely originated as glovebox floor sweepings and oxide screenings from metal-to-oxide conversion. MIS item C06032A likely originated in pyrochemical operations. The PG results for the materials packaged in 3013 container shows that all of the containers have chloride bearing oxide, which is likely because of the mixing of multiple items during 3013 packaging.

- MIS items considered but not used for representation: none.

\section{Oxide from Hydroxide Precipitation Rocky Flats}

- 3013 Taxon: MgOHPPt-RF

- Formerly: Rocky Flats 2F

- Number of 3013 Containers: 81

- Representation in MIS

\begin{tabular}{|c|c|c|}
\hline Original & Add/Remove/Keep & Current \\
\hline 39-01153A & Keep & $39-01153 A^{\dagger}$ \\
\hline \multicolumn{2}{|c|}{ Add } & $66-00-11-355 *^{\dagger}$ \\
\hline \multicolumn{2}{|c|}{ Add } & $66-01-01-439 *^{\dagger}$ \\
\hline $\begin{array}{r}\text { *Small-Scale Surveillance } \\
\text { tFull Characterization, including chemistry, surface area, density, etc. }\end{array}$
\end{tabular}

- This group consists of plutonium and uranium oxides that were produced from the magnesium hydroxide precipitation process. ${ }^{4}$ This process was used to process plutonium and uranium solutions as well as holdup solutions in tanks and pipes during cleanup operations.

- The assay values for this group range from 33.4 to $92.6 \mathrm{wt} \%$ actinide. ${ }^{8}$ The plutonium assay ranges from 19.8 to $74.6 \mathrm{wt} \%$. Uranium is present in ten containers and is found in concentrations of up to $66.2 \mathrm{wt} \%$.

- PG analysis was completed for all 81 containers. All 81 containers are classified "other impurities," including aluminum, beryllium, magnesium, and sodium. Magnesium is the largest impurity and ranges in concentration from 1.4 to 22.6 wt\%. Beryllium was found in one container at $0.08 \mathrm{wt} \%$.

- The only MIS item from Rocky Flats (39-01153A) intended to represent this group has only $7.7 \mathrm{wt} \%$ plutonium with no uranium, and is not very representative of this material. Therefore, Hanford samples from the same process were included to represent this material.

- MIS items considered but not used for representation: None. 


\section{Rocky Flats Oxide from Metal Oxidation at Savannah River}

- 3013 Taxon: MetalOx-SR-RF

- Formerly: Savannah River 1A

- Number of 3013 Containers: 72

- Representation in MIS

\begin{tabular}{|c|c|c|}
\hline Original & Add/Remove/Keep & Current \\
\hline TS707001 & Keep & $\mathrm{TS}^{2} 07001^{*^{\dagger}}$ \\
\hline 011608 & Keep & $011608^{\dagger}$ \\
\hline 011589A & Keep & $011589 A^{* \dagger \mp}$ \\
\hline ARF-102-85-114-1 & Keep & ARF-102-85-114-1* ${ }^{\dagger}$ \\
\hline & Add & $07221730^{\dagger}$ \\
\hline
\end{tabular}

- This group of containers consists of Rocky Flats material that is mostly foundry oxide sent to Savannah River Site for recovery during the 1980 s. $^{5}$ This group also includes plutonium/uranium oxides from hydride oxidation or residue processing and from the decontamination of metal parts.

- The assay values for this group range from 75.1 to $94.2 \mathrm{wt} \%$ actinide. ${ }^{8}$ The plutonium assay ranges from 17.0 to $88.9 \mathrm{wt} \%$. Uranium is present in 12 containers and ranges in concentration of up to $77.3 \mathrm{wt} \%$.

- $\quad$ PG analysis was completed for 71 containers. Chlorine was found in 16 containers and ranges in concentration from 0.25 to $1.2 \mathrm{wt} \%$. Fluorine (without chlorine) was found in 36 containers and ranges in concentration from 0.02 to $1.4 \mathrm{wt} \%$. Prompt gamma did not detect impurities in ten containers, and the remaining nine containers have "other impurities," including aluminum, beryllium, magnesium, and sodium. Beryllium was found in ten containers, and ranges from 0.02 to $0.9 \mathrm{wt} \%$.

- Three Rocky Flats samples and one Rocky Flats metal-oxidation sample from Hanford were originally selected for representation. MIS item 011589A represents only certain containers determined to be either potentially (7) or most similar (7). MIS Item 07221730, a Rocky Flats sample from metal oxidation was added to the group because it is a high-purity oxide that originated in metal oxidation at Rocky Flats. 
- MIS items considered but not used for representation:

\begin{tabular}{|c|c|}
\hline MIS Item & $\begin{array}{c}\text { Reason not used } \\
\end{array}$ \\
\hline 053038 & $\begin{array}{l}\text { Based on the chemical characterization, this } \\
\text { material has impurities that are unlike those found } \\
\text { in other MIS items in this group. }\end{array}$ \\
\hline PMAXBS & $\begin{array}{l}\text { This is a LANL-generated material produced by } \\
\text { mixing anode heel with ER salt, and may have } \\
\text { different impurities from those expected for this } \\
\text { group. }\end{array}$ \\
\hline 101707001 & $\begin{array}{l}\text { This MIS item contains less than } 30 \mathrm{wt} \% \text { actinide. } \\
\text { This sample was also not characterized in the MIS } \\
\text { program and is not included in small-scale, shelf-life } \\
\text { experiments. }\end{array}$ \\
\hline TS707013 & $\begin{array}{l}\text { This Rocky Flats metal-oxidation sample is high in } \\
\text { chloride and outside the range of actinide } \\
\text { concentration. }\end{array}$ \\
\hline $\begin{array}{l}\text { ARF-102-85-223 } \\
\text { ARF-102-85-295 } \\
\text { ARF-102-85-355 } \\
\text { ARF-102-85-365 }\end{array}$ & $\begin{array}{l}\text { These MIS items of Rocky Flats material at Hanford } \\
\text { are high in chloride and outside the range of } \\
\text { actinide concentration. }\end{array}$ \\
\hline
\end{tabular}

\section{Oxide from Oxalate Precipitation at Savannah River}

- 3013 Taxon: OxlPPt-SR

- Formerly: Savannah River 1C

- Number of 3013 Containers: 112

- Representation in MIS

\begin{tabular}{|c|c|c|}
\hline Original & Add/Remove/Keep & Current \\
\hline PBO-47-09-012-023 & Keep & PBO-47-09-012-023* \\
\hline BLO-39-11-14-004 & Keep & BLO-39-11-14-004* ${ }^{\dagger}$ \\
\hline & Add & PEOF1 ${ }^{*^{\dagger}}$ \\
\hline
\end{tabular}

- This group of containers consists of high purity oxide from oxalate precipitation from purified nitrate from HB-Line and Hanford. ${ }^{5}$

- The assay values for this group are all greater than $85 \mathrm{wt} \%$ actinide. ${ }^{8}$ The plutonium assay ranges from 85.1 to $95 \mathrm{wt} \%$. Uranium is not found in any containers.

- PG analysis was completed for 89 containers. Chlorine was not detected in any containers. Fluorine was found in two containers around $0.2 \mathrm{wt} \%$. Prompt gamma did not detect impurities in 63 containers, and the remaining 24 containers have only low concentrations of sodium $(<0.02 \mathrm{wt} \%)$. Beryllium was not detected in any containers.

- All three samples representing this group are high-purity samples from oxalate precipitation. MIS item PEOF1 was added because it was generated by the same process and has a similar assay to the materials in this group. 
- MIS items considered but not used for representation:

\begin{tabular}{|c|l|}
\hline MIS Item & \multicolumn{1}{c|}{ Reason not used } \\
\hline MISSTD-1 & $\begin{array}{l}\text { This MIS item was not stabilized at } 950^{\circ} \mathrm{C} \text { and has } \\
\text { a high surface area, which is not expected to be } \\
\text { representative of these materials. }\end{array}$ \\
\hline MISSTD-2 & $\begin{array}{l}\text { This MIS item was not characterized and was not } \\
\text { included in small-scale, shelf-life experiments. }\end{array}$ \\
\hline CXLNM1 & $\begin{array}{l}\text { This MIS item was not characterized and was not } \\
\text { included in small-scale, shelf-life experiments. In } \\
\text { addition, the material in this sample was } \\
\text { precipitated from chloride instead of nitrate. }\end{array}$ \\
\hline PEOR3258 & $\begin{array}{l}\text { This MIS item has limited characterization data and } \\
\text { was not included in small-scale, shelf-life } \\
\text { experiments. }\end{array}$ \\
\hline ATL27960 & $\begin{array}{l}\text { This MIS item was not well characterized by } \\
\text { process history or analytical chemistry, and material } \\
\text { is no longer available in the MIS inventory. In } \\
\text { addition, this MIS item has an assay value outside } \\
\text { of the range for this group. }\end{array}$ \\
\hline
\end{tabular}

\section{LLNL Metal Oxidation at Savannah River}

- 3013 Taxon: MetalOx-SR-LL

- Formerly: Savannah River 1D

- Number of 3013 Containers: 35

- Representation in MIS:

\begin{tabular}{|c|c|c|}
\hline Original & Add/Remove/Keep & Current \\
\hline TS707001 & Keep & TS707001* ${ }^{\dagger}$ \\
\hline 011608 & Keep & $011608^{\dagger}$ \\
\hline 011589A & Remove & \\
\hline \multirow[t]{4}{*}{ MT1490 } & Keep & MT1490* ${ }^{\dagger}$ \\
\hline & Add & CXL1685 ${ }^{\dagger}$ \\
\hline & Add & $07221730^{\dagger}$ \\
\hline & Add & ARF-102-85-114-1* \\
\hline
\end{tabular}

- This group of containers consists of plutonium metal from LLNL that had been burned to metal. $^{5}$

- The assay values for this group range from 66.9 to $90.8 \mathrm{wt} \%$ actinide. ${ }^{8}$ The plutonium assay ranges from 10.5 to $90.8 \mathrm{wt} \%$. Uranium is found in five containers and ranges from 27.1 to $75.5 \mathrm{wt} \%$.

- PG analysis was completed for 32 containers. One container has chlorine at $2 \mathrm{wt} \%$, and two containers have fluorine at 0.02 and $0.2 \mathrm{wt} \%$. Prompt gamma did not detect impurities in 24 containers, and the remaining five containers have "other impurities" including aluminum and sodium. Beryllium was not detected in any containers. 
- This group is represented by pure metal oxidation items from Rocky Flats. MIS item 011589A does not represent any containers in this group, based on the process history. ${ }^{10}$ MIS item CXL1685 was included to represent those containers with high uranium concentrations, and MIS items 07221730 and ARF-102-85-

114-1 were added because they consist of high-purity oxide from metal oxidation.

- MIS items considered but not used for representation:

\begin{tabular}{|c|l|}
\hline MIS Item & \multicolumn{1}{c|}{ Reason not used } \\
\hline 101707001 & $\begin{array}{l}\text { This MIS item contains less than 30 wt\% actinide. } \\
\text { This sample was also not characterized in the MIS } \\
\text { program and is not included in small-scale, shelf-life } \\
\text { experiments. }\end{array}$ \\
\hline TS707013 & $\begin{array}{l}\text { This Rocky Flats metal oxidation sample is high in } \\
\text { chloride and is outside the range of actinide } \\
\text { concentration. }\end{array}$ \\
\hline 011589A & $\begin{array}{l}\text { This MIS item exhibited unique behavior in small- } \\
\text { scale, shelf-life experiments that is not considered } \\
\text { likely in these materials, based on process history. }\end{array}$ \\
\hline
\end{tabular}

\section{Oxide from Metal Brushings at Savannah River}

- 3013 Taxon: MetalOx-SR

- Formerly: Savannah River 1E

- Number of 3013 Containers: 5

Representation in MIS:

\begin{tabular}{|c|c|c|}
\hline Original & Add/Remove/Keep & Current \\
\hline TS707001 & Keep & TS707001 $^{{ }^{\dagger}}$ \\
\hline 011608 & Keep & $011608^{\dagger}$ \\
\hline $011589 \mathrm{~A}$ & Remove & $07221730^{\dagger}$ \\
\hline \multicolumn{2}{|c|}{ Add } & ARF-102-85-114-1 $^{{ }^{\dagger}}$ \\
\hline \multicolumn{2}{|c|}{} & Add \\
\hline *Small-Scale Surveillance \\
tFull Characterization, including chemistry, surface area, density, etc.
\end{tabular}

- The materials in this group consist of metal brushings that were generated during the packaging of metal in 3013 containers. ${ }^{5}$

- The actinide and plutonium assay values for this group range from 71.0 to greater than $86.6 \mathrm{wt} \%$ actinide. ${ }^{8}$ The plutonium assay ranges from 71.0 to $86.6 \mathrm{wt} \%$. Uranium is present in one container at $0.26 \mathrm{wt} \%$.

- PG analysis was completed for all five containers. All five containers show fluoride and magnesium without chloride. The fluorine concentration ranges from 0.3 to $3.9 \mathrm{wt} \%$, and the magnesium concentration ranges from 0.04 to $0.4 \mathrm{wt} \%$. Beryllium was not detected in any containers.

- This group is represented by pure metal oxidation items from Rocky Flats. MIS item 011589A does not represent any containers in this group because chloride was not detected. MIS items 07221730 and ARF-102-85-114-1 were added because they consist of high-purity oxide from metal oxidation. 
- MIS items considered but not used for representation:

\begin{tabular}{|c|l|}
\hline MIS Item & \multicolumn{1}{|c|}{ Reason not used } \\
\hline 101707001 & $\begin{array}{l}\text { This MIS item contains less than } 30 \text { wt\% actinide. } \\
\text { This sample was also not characterized in the MIS } \\
\text { program and is not included in small-scale, shelf-life } \\
\text { experiments. }\end{array}$ \\
\hline TS707013 & $\begin{array}{l}\text { This Rocky Flats metal oxidation sample is high in } \\
\text { chloride and outside the range of actinide } \\
\text { concentration. }\end{array}$ \\
\hline 011589A & $\begin{array}{l}\text { This MIS item exhibited unique behavior in small- } \\
\text { scale, shelf-life experiments that is not considered } \\
\text { likely in these materials, based on process history } \\
\text { and lack of chloride. }\end{array}$ \\
\hline
\end{tabular}

\section{Hanford Mixed Oxides at Savannah River}

- 3013 Taxon: PuUOx-SR-HN

- Formerly: Savannah River 1F

- Number of 3013 Containers: 30

- Representation in MIS:

\begin{tabular}{|c|c|c|}
\hline Original & Add/Remove/Keep & Current \\
\hline SCP711-46 & Keep & SCP711-46 \\
\hline SCP711-56 & Keep & SCP711-56 \\
\hline MOXSCP-COM & Remove & \\
\hline & Add & $5501407^{*^{\dagger}}$ \\
\hline & Add & $669194^{{ }^{\dagger}}$ \\
\hline \multicolumn{2}{|c|}{ Add } & CAN92 $^{{ }^{\dagger}}$ \\
\hline & Add & CXL1685 \\
\hline $\begin{array}{l}\text { *Small-Scale Surveillance } \\
\text { tFull Characterization, including chemistry, surface area, density, etc. }\end{array}$ \\
\hline
\end{tabular}

- This group of containers consists of mixed reactor fuel product. The plutonium oxide was oxalate derived. ${ }^{5}$

- The assay values show that the materials in this group are greater than $80.9 \mathrm{wt} \%$ actinide. ${ }^{8}$ The plutonium assay ranges from 16.6 to $33.8 \mathrm{wt} \%$. Uranium is found in all 30 containers and ranges from 47.1 to $83.3 \mathrm{wt} \%$.

- A PG analysis was completed for all 30 containers. Chlorine, fluorine, and beryllium were not detected. Eleven containers have "other impurities," including aluminum, magnesium, and sodium; and the remaining 19 containers have no impurities detected.

- This group is represented by pure mixed plutonium/uranium oxides from LANL. Other mixed oxides from RFETS and LANL, including 5501407, 669194, CAN92, and CXL1685, were added to this group, based on process similarities. 
- MIS items considered but not used for representation:

\begin{tabular}{|c|c|}
\hline MIS Item & $\begin{aligned} \text { Reason not used } \\
\end{aligned}$ \\
\hline MOXSCP-COM & $\begin{array}{l}\text { This MIS item, although similar in actinide content, } \\
\text { was not included because it was not characterized } \\
\text { and not included in small-scale, shelf-life } \\
\text { experiments. }\end{array}$ \\
\hline $\begin{array}{l}\text { CXL-BMU Mixtures } \\
(1685, \text { BMU, } \\
\text { BMUCXL70-30, } \\
\text { BMUCXL93-7, } \\
\text { CXLBMU70-30, and } \\
\text { CXLBMU93-7) }\end{array}$ & $\begin{array}{l}\text { These MIS items were used for neutron moderation } \\
\text { and moisture experiments and were not intended } \\
\text { for representation. Therefore, they were not } \\
\text { characterized and not included in small-scale, shelf- } \\
\text { life experiments. }\end{array}$ \\
\hline $\begin{array}{l}\text { Pure uranium oxide } \\
\text { items: } 1685 \text { and BMU }\end{array}$ & $\begin{array}{l}\text { These MIS items were used for neutron moderation } \\
\text { experiments and not intended for representation. } \\
\text { Therefore, they were not included in small-scale } \\
\text { surveillance experiments. }\end{array}$ \\
\hline PSU-84-06-05 & $\begin{array}{l}\text { The material in this sample is considered specific to } \\
\text { pyrolytic processing of polycubes and is not } \\
\text { considered representative of this material. }\end{array}$ \\
\hline MISNE2 & $\begin{array}{l}\text { This MIS item is a mixture of Rocky Flats items } \\
\text { from various processes, which may have introduced } \\
\text { impurities that are not representative of this } \\
\text { process. In addition, gypsum and borax were added } \\
\text { to this material for neutron moderation experiments. }\end{array}$ \\
\hline 053038 & $\begin{array}{l}\text { This MIS item has a high chloride content, but } \\
\text { chloride is not present in these materials. }\end{array}$ \\
\hline PuUOXBC05 & $\begin{array}{l}\text { This MIS item, although a mixed oxide, is not } \\
\text { completely characterized, and its process history is } \\
\text { unknown. This sample was also not included in } \\
\text { small-scale, shelf-life experiments. }\end{array}$ \\
\hline
\end{tabular}

\section{Mixed Oxides from Alloy Oxidation at Savannah River}

- 3013 Taxon: PuUOx-SR-Alloy

- Formerly: Savannah River 2A

- Number of 3013 Containers: 17

- Representation in MIS:

\begin{tabular}{|c|c|c|}
\hline Original & Add/Remove/Keep & Current \\
\hline 5501407 & Keep & $5501407^{*^{\dagger}}$ \\
\hline $011589 \mathrm{~A}$ & Remove & \\
\hline & Add & $669194^{{ }^{\dagger}}$ \\
\hline & Add & CXL1685 $^{\dagger}$ \\
\hline \multicolumn{2}{|c|}{ Add } & SCP711-46 $^{\dagger}$ \\
\hline & Add & SCP711-56 $^{{ }^{\dagger}}$ \\
\hline *Small-Scale Surveillance \\
†Full Characterization, including chemistry, surface area, density, etc.
\end{tabular}


- This group of containers consists of oxide produced from the oxidation of Pu/EU, $\mathrm{Pu} / \mathrm{DU} / \mathrm{Mo}$, and $\mathrm{Pu} / \mathrm{EU} / \mathrm{Zr}$ alloys from Argonne. ${ }^{5}$

- The assay values for this group range from 64.2 to $96.7 \mathrm{wt} \%$ actinide. ${ }^{8}$ The plutonium assay ranges from 11.2 to $32.6 \mathrm{wt} \%$. Uranium is present in all 17 containers and ranges from 46.7 to $85.5 \mathrm{wt} \%$.

- A PG analysis was completed for all 17 containers. Chlorine was not detected in these materials. One container has fluorine at $0.3 \mathrm{wt} \%$. Six containers have "other impurities," which include aluminum, magnesium, and sodium, and the remaining ten containers have no impurities detected.

- This group was originally represented by MIS items 5501407, a mixed oxide from Rocky Flats, and 011589A. MIS item 011589A does not represent any containers in this group because chloride was not detected. Additional MIS items, including 669194, CXL1685, SCP711-46, and SCP711-56, were matched to this group, based on their composition and plutonium and uranium assay ranges. 
- MIS items considered but not used for representation:

\begin{tabular}{|c|c|}
\hline MIS Item & Reason not used \\
\hline MOXSCP-COM & $\begin{array}{l}\text { This MIS item, although similar in actinide content, } \\
\text { was not included because it was not characterized } \\
\text { and not included in small-scale, shelf-life } \\
\text { experiments. }\end{array}$ \\
\hline $\begin{array}{l}\text { CXL-BMU Mixtures } \\
\text { (1685, BMU, } \\
\text { BMUCXL70-30, } \\
\text { BMUCXL93-7, } \\
\text { CXLBMU70-30, and } \\
\text { CXLBMU93-7) }\end{array}$ & $\begin{array}{l}\text { These MIS items were used for neutron moderation } \\
\text { and moisture experiments and were not intended } \\
\text { for representation. Therefore, they were not } \\
\text { characterized and not included in small-scale, shelf- } \\
\text { life experiments. }\end{array}$ \\
\hline $\begin{array}{l}\text { Pure uranium oxide } \\
\text { items: } 1685 \text { \& BMU }\end{array}$ & $\begin{array}{l}\text { These MIS items were used for neutron moderation } \\
\text { experiments and not intended for representation. } \\
\text { Therefore, they were not included in small-scale } \\
\text { surveillance experiments. }\end{array}$ \\
\hline PSU-84-06-05 & $\begin{array}{l}\text { The material in this sample is considered specific to } \\
\text { pyrolytic processing of polycubes and is not } \\
\text { considered representative of this material. }\end{array}$ \\
\hline MISNE2 & $\begin{array}{l}\text { This MIS item is a mixture of Rocky Flats items } \\
\text { from various processes, which may have introduced } \\
\text { impurities that are not representative of this } \\
\text { process. In addition, gypsum and borax were added } \\
\text { to this material for neutron moderation experiments. }\end{array}$ \\
\hline 053038 & $\begin{array}{l}\text { This MIS item has a high chloride content, but } \\
\text { chloride is not present in these materials. }\end{array}$ \\
\hline PuUOXBC05 & $\begin{array}{l}\text { This MIS item, although a mixed oxide, is not } \\
\text { completely characterized, and its process history is } \\
\text { unknown. This sample was also not included in } \\
\text { small-scale, shelf-life experiments. }\end{array}$ \\
\hline CAN92 & $\begin{array}{l}\text { The uranium concentration is below the range for } \\
\text { this group of material. In addition, this MIS item } \\
\text { contains beryllium, but beryllium was not detected } \\
\text { in any } 3013 \text { containers in this group. }\end{array}$ \\
\hline 011589A & $\begin{array}{l}\text { This MIS item exhibited unique behavior in small- } \\
\text { scale, shelf-life experiments that is not considered } \\
\text { likely in these materials, based on process history } \\
\text { and lack of chloride. }\end{array}$ \\
\hline
\end{tabular}

\section{Scrap Mixed Oxides at Savannah River}

- 3013 Taxon: PuUOx-SR-Misc

- Formerly: Savannah River 2B

- Number of 3013 Containers: 27 
- Representation in MIS:

\begin{tabular}{|c|c|c|}
\hline Original & Add/Remove/Keep & Current \\
\hline 62750 & Remove & \\
\hline CAN92 & Keep & CAN92* ${ }^{\dagger}$ \\
\hline 669194 & Keep & $669194^{\star^{\dagger}}$ \\
\hline 053038 & Remove & \\
\hline 5501407 & Keep & $5501407^{*^{\dagger}}$ \\
\hline ATL27960 & Remove & \\
\hline \multirow{3}{*}{ CXL1685 } & Keep & CXL1685 ${ }^{\dagger}$ \\
\hline & Add & $\mathrm{SCP}^{2} 11-46^{\dagger}$ \\
\hline & Add & SCP711-56* \\
\hline
\end{tabular}

- This group of containers consists of plutonium oxide and mixed plutonium/uranium oxides from various sites, including LANL, Hanford, SRS, Oak Ridge National Laboratory (ORNL). ${ }^{5}$ This group may contain co-precipitated material produced through the Kerr-McGee process.

- The assay values show that the materials in this group are greater than $57.1 \mathrm{wt} \%$ actinide. ${ }^{8}$ The plutonium assay ranges from 7.5 to $83.7 \mathrm{wt} \%$. Uranium is present in 16 containers and is found in concentrations ranging from 1.4 to $85.3 \mathrm{wt} \%$.

- PG analysis was completed for 26 containers. One container has chlorine at 0.7 $\mathrm{wt} \%$. Fluorine (without chlorine) was found in ten containers and ranges in concentration from 0.06 to11.7 wt\%. Prompt gamma did not detect impurities in eight containers, and the remaining seven containers have "other impurities," including aluminum, beryllium, magnesium, and sodium. Beryllium was detected in three containers and ranges from 0.05 to $0.1 \mathrm{wt} \%$.

- This group is represented by mixed plutonium/uranium oxides from Rocky Flats and LANL. MIS items selected include mixed plutonium/uranium oxides that match the respective plutonium and uranium assay ranges. Additional MIS items, including SCP711-46, and SCP711-56, were matched to this group, based on their composition and plutonium and uranium assay ranges. 
- MIS items considered but not used for representation:

\begin{tabular}{|c|c|}
\hline MIS Item & Reason not used \\
\hline MOXSCP-COM & $\begin{array}{l}\text { This MIS item, although similar in actinide content, } \\
\text { was not included because it was not characterized } \\
\text { and not included in small-scale, shelf-life } \\
\text { experiments. }\end{array}$ \\
\hline $\begin{array}{l}\text { CXL-BMU Mixtures } \\
\text { (1685, BMU, } \\
\text { BMUCXL70-30, } \\
\text { BMUCXL93-7, } \\
\text { CXLBMU70-30, and } \\
\text { CXLBMU93-7) }\end{array}$ & $\begin{array}{l}\text { These MIS items were used for neutron moderation } \\
\text { and moisture experiments and not intended for } \\
\text { representation. Therefore, they were not } \\
\text { characterized and not included in small-scale, shelf- } \\
\text { life experiments. }\end{array}$ \\
\hline $\begin{array}{l}\text { Pure uranium oxide } \\
\text { items: } 1685 \text { \& BMU }\end{array}$ & $\begin{array}{l}\text { These MIS items were used for neutron moderation } \\
\text { experiments and not intended for representation. } \\
\text { Therefore, they were not included in small-scale } \\
\text { surveillance experiments. }\end{array}$ \\
\hline PSU-84-06-05 & $\begin{array}{l}\text { The material in this sample is considered specific to } \\
\text { pyrolytic processing of polycubes and is not } \\
\text { considered representative of this material. }\end{array}$ \\
\hline MISNE2 & $\begin{array}{l}\text { This MIS item is a mixture of Rocky Flats items } \\
\text { from various processes, which may have introduced } \\
\text { impurities that are not representative of this } \\
\text { process. In addition, gypsum and borax were added } \\
\text { to this material for neutron moderation experiments. }\end{array}$ \\
\hline 053038 & $\begin{array}{l}\text { This MIS item has high chloride content, but } \\
\text { chloride is not present in high concentrations in } \\
\text { these materials. }\end{array}$ \\
\hline PuF4-1 & $\begin{array}{l}\text { PuF4-1 was considered for representation because } \\
\text { of the high fluoride concentrations found in some } \\
3013 \text { containers in this group. However, the } \\
\text { material is not from the same process and may not } \\
\text { be in the same chemical form. }\end{array}$ \\
\hline 62750 & $\begin{array}{l}\text { This MIS item is outside of the range for uranium, } \\
\text { and this material is a dissolution residue, which is } \\
\text { not expected to be representative of this material. }\end{array}$ \\
\hline ATL27960 & $\begin{array}{l}\text { This MIS item was not well characterized by } \\
\text { process history or analytical chemistry, and material } \\
\text { is no longer available in the MIS inventory. In } \\
\text { addition, this MIS item has an assay value outside } \\
\text { of the range for this group. }\end{array}$ \\
\hline
\end{tabular}

\section{Oxide Precipitation from Chloride Solution at LANL}

- 3013 Taxon: OxlPPt-LA-Cl

- Formerly: N/A

- Number of 3013 Containers: 116 estimated 
- Represented in MIS:

\begin{tabular}{|c|c|c|}
\hline Original & Add/Remove/Keep & Current \\
\hline CXLOX091802 & Keep & CXLOX091802**†† \\
\hline CXLPROD091901 & Keep & CXLPROD091901**†† \\
\hline CXLPROD021202 & Keep & CXLPROD021202**†T \\
\hline & Add & CXLNM1 \\
\hline
\end{tabular}

- This group of materials was generated by the oxalate precipitation process from aqueous chloride solutions. ${ }^{7}$ Most of the material is newly generated oxide, but this group also includes material from historical aqueous chloride processes.

- The plutonium assay for these materials is expected to range from 58 to $88 \mathrm{wt} \%$. Most of the material is in the range from 75 to $88 \mathrm{wt} \%$ plutonium. Significant quantities of uranium are not expected in these materials.

- The MIS items representing this group were produced by the oxalate precipitation process from aqueous chloride solutions. The impurities in these materials are expected to be different than those found in material generated by oxalate precipitation from nitrate solutions. The difference in the items with the prefix CXLOX and CXLPROD is that the CXLPROD materials underwent solvent extraction before oxalate precipitation and are higher in purity than the CXLOX materials.

\section{Oxide Precipitation from Nitrate Solutions at LANL}

- 3013 Taxon: OxlPPt-LA-NO3

- Formally: N/A

- Number of 3013 Containers: 25 estimated

- Represented in MIS

\begin{tabular}{|c|c|c|}
\hline Original & Add/Remove/Keep & Current \\
\hline PEOF1 & Keep & PEOF1 $^{*^{\dagger}}$ \\
\hline PEOR3258 & Keep & PEOR3258 $^{\dagger}$ \\
\hline BLO-39-11-14-004 & Keep & BLO-39-11-14-004 $^{*^{\dagger}}$ \\
\hline PBO-47-09-012-023 & Keep & PBO-47-09-012-023 \\
\hline $\begin{array}{l}\text { *Small-Scale Surveillance } \\
\text { tFull Characterization, including chemistry, surface area, density, etc. }\end{array}$ \\
\hline
\end{tabular}

- This group of materials was generated by the oxalate precipitation process from aqueous nitrate solutions. ${ }^{7}$ Most of the material is newly generated oxide, but this group also includes material from historical aqueous nitrate processes.

- The plutonium assay for these materials is expected to range from 64 to $88 \mathrm{wt} \%$. Most of the material is in the range from 75 to $88 \mathrm{wt} \%$ plutonium. Significant quantities of uranium are not expected in these materials.

- The MIS items representing this group were produced by the oxalate precipitation process from aqueous nitrate solutions at LANL and Hanford. 


\section{Dissolution Residues at LANL}

- 3013 Taxon: DisResd-LA

- Formally: N/A

- Number of 3013 Containers: 6 estimated

- Represented in MIS:

\begin{tabular}{|c|c|c|}
\hline Original & Add/Remove/Keep & Current \\
\hline 62750 & Keep & $62750^{\dagger}$ \\
\hline 07032282A & Keep & $07032282 A^{*^{\dagger}}$ \\
\hline $07242201 \mathrm{~A}$ & Keep & $07242201 A^{*^{\dagger}}$ \\
\hline 07242165A & Keep & $07242165 A^{*^{\dagger}}$ \\
\hline ARF-102-85-355 & Keep & ARF-102-85-355* \\
\hline New Sample & To be added & New Sample \\
\hline
\end{tabular}

- This group consists of insoluble materials remaining after dissolution in the aqueous nitrate and chloride processes. ${ }^{7}$ This group includes both newly generated and historical materials from this process.

- The plutonium assay is expected to range from 30 to $88 \mathrm{wt} \%$.

- MIS items representing this group include Rocky Flats dissolution residues from the aqueous nitrate process and a new MIS item from chloride dissolution that will be added to the inventory.

\section{Metal Oxidation at $L A N L$}

- 3013 Taxon: MetalOx-LA

- Formally: N/A

- Number of 3013 Containers: 42 estimated

- Represented in MIS:

\begin{tabular}{|c|c|c|}
\hline Original & Add/Remove/Keep & Current \\
\hline MT1490 & Keep & MT1490 $^{*^{\dagger}}$ \\
\hline 011608 & Keep & $011608^{\dagger}$ \\
\hline 07221730 & Keep & $07221730^{\dagger}$ \\
\hline ARF-102-85-114-1 & Keep & ARF-102-85-114-1 ${ }^{{ }^{\dagger}}$ \\
\hline TS707001 & Keep & TS707001 $^{*^{\dagger}}$ \\
\hline TS707013 & Keep & TS707013 $^{*^{\dagger}}$ \\
\hline $\begin{array}{l}\text { *Small-Scale Surveillance } \\
\text { tFull Characterization, including chemistry, surface area, density, etc. }\end{array}$ \\
\hline
\end{tabular}

- This group consists of oxide produced in metal oxidation processes. ${ }^{7}$ Alloyed and unalloyed metal items from various processes, including electrorefining, pyrochemical, and unknown sources, will be burned to oxide.

- The plutonium assay is expected to range from 30 to $88 \mathrm{wt} \%$.

- This group is represented by metal oxidation items from Rocky Flats. 


\section{Plutonium/Uranium Oxide at LANL}

- 3013 Taxon: PuUOx-LA

- Formally: N/A

- Number of 3013 Containers: 12 estimated

- Represented in MIS:

\begin{tabular}{|c|c|c|}
\hline Original & Add/Remove/Keep & Current \\
\hline SCP711-46 & Keep & SCP711-46 \\
\hline PuUOXBC05 & Keep & PuUOXBC05 \\
\hline SCP711-56 & Keep & SCP711-56 ${ }^{{ }^{\dagger}}$ \\
\hline *Small-Scale Surveillance \\
tFull Characterization, including chemistry, surface area, density, etc.
\end{tabular}

- This group consists of mixed oxides of plutonium and uranium. ${ }^{7}$

- The plutonium assay is expected to range from 10 to $79 \mathrm{wt} \%$, and the uranium assay is expected to range from 0 to $73 \mathrm{wt} \%$.

- This group is represented by mixed oxides of plutonium and uranium produced at LANL.

\section{Pyrochemical Oxides at LANL}

- 3013 Taxon: PyroOx-LA

- Formally: N/A

- Number of 3013 Containers: 9 estimated

- Represented in MIS:

\begin{tabular}{|c|c|c|}
\hline Original & Add/Remove/Keep & Current \\
\hline ATL27960 & Keep & ATL27960 \\
\hline C00695 & Keep & $\mathrm{C} 00695^{\dagger}$ \\
\hline CLLANL025 & Keep & CLLANL025* $^{\dagger}$ \\
\hline PMAXBS & Keep & $\mathrm{PMAXBS}^{*^{\dagger}}$ \\
\hline 520610020 & Keep & $520610020^{*^{\dagger}}$ \\
\hline C00024A & Keep & 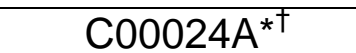 \\
\hline ARF-102-85-223 & Keep & ARF-102-85-223* ${ }^{\top}$ \\
\hline ARF-102-85-295 & Keep & ARF-102-85-295* ${ }^{\dagger}$ \\
\hline ARF-102-85-365 & Keep & ARF-102-85-365* \\
\hline
\end{tabular}

- This group consists of plutonium oxide from pyrochemical processes at LANL. ${ }^{7}$ The materials consist of anode heel, electrorefining scrap, and other pyrochemical oxide from unknown sources.

- The plutonium assay is expected to range from 80 to $88 \mathrm{wt} \%$.

- This group is represented by pyrochemical materials generated at Rocky Flats and LANL. 
Appendix D: Master PG Represented Table 


\begin{tabular}{|c|c|c|}
\hline $\begin{array}{c}3013 \\
\text { Container }\end{array}$ & $\begin{array}{l}\text { MIS Represented Item } \\
\text { Selection Order }\end{array}$ & $\begin{array}{c}\text { MIS Represented } \\
\text { Item }\end{array}$ \\
\hline \multirow[t]{3}{*}{ H000808 } & PGCL-1-Cl\% & ARF-102-85-295 \\
\hline & PGCL-2-Cl\% & CLLANL025 \\
\hline & PGCL-3-Cl\% & C00024A \\
\hline \multirow[t]{3}{*}{$\mathrm{H} 000817$} & PGCL-1-CI\% & ARF-102-85-365 \\
\hline & PGCL-2-Cl\% & 053038 \\
\hline & PGCL-3-Cl\% & TS707001 \\
\hline \multirow[t]{3}{*}{$\mathrm{H} 000849$} & PGCL-1-Cl\% & 053038 \\
\hline & PGCL-2-Cl\% & ARF-102-85-365 \\
\hline & PGCL-3-Cl\% & TS707001 \\
\hline \multirow[t]{3}{*}{ H000913 } & PGCL-1-Cl\% & 053038 \\
\hline & PGCL-2-Cl\% & ARF-102-85-365 \\
\hline & PGCL-3-CI\% & TS707001 \\
\hline \multirow[t]{3}{*}{$\mathrm{H} 000914$} & PGCL-1-Cl\% & TS707001 \\
\hline & PGCL-2-Cl\% & ARF-102-85-365 \\
\hline & PGCL-3-Cl\% & 053038 \\
\hline \multirow[t]{3}{*}{ H000929 } & PGCL-1-Cl\% & ARF-102-85-365 \\
\hline & PGCL-2-Cl\% & 053038 \\
\hline & PGCL-3-Cl\% & TS707001 \\
\hline \multirow[t]{3}{*}{$\mathrm{H} 000937$} & PGCL-1-Cl\% & TS707001 \\
\hline & PGCL-2-Cl\% & ARF-102-85-365 \\
\hline & PGCL-3-Cl\% & 053038 \\
\hline \multirow[t]{3}{*}{$\mathrm{H} 003556$} & PGCL-1-Cl\% & 053038 \\
\hline & PGCL-2-Cl\% & ARF-102-85-365 \\
\hline & PGCL-3-Cl\% & TS707001 \\
\hline \multirow[t]{3}{*}{ H003589 } & PGCL-1-Cl\% & TS707001 \\
\hline & PGCL-2-Cl\% & ARF-102-85-365 \\
\hline & PGCL-3-Cl\% & 053038 \\
\hline \multirow[t]{3}{*}{ L000076 } & PGCL-1-Cl\% & ARF-102-85-365 \\
\hline & PGCL-2-Cl\% & 053038 \\
\hline & PGCL-3-Cl\% & TS707001 \\
\hline \multirow[t]{3}{*}{ L000155 } & PGCL-1-Cl\% & 053038 \\
\hline & PGCL-2-Cl\% & ARF-102-85-365 \\
\hline & PGCL-3-Cl\% & TS707001 \\
\hline \multirow[t]{3}{*}{ L000167 } & PGCL-1-Cl\% & PMAXBS \\
\hline & PGCL-2-Cl\% & ARF-102-85-223 \\
\hline & PGCL-3-Cl\% & $\mathrm{C} 00695$ \\
\hline \multirow[t]{3}{*}{ L000181 } & PGCL-1-Cl\% & ARF-102-85-365 \\
\hline & PGCL-2-Cl\% & 053038 \\
\hline & PGCL-3-Cl\% & TS707001 \\
\hline \multirow[t]{3}{*}{ L000204 } & PGCL-1-Cl\% & 053038 \\
\hline & PGCL-2-Cl\% & ARF-102-85-365 \\
\hline & PGCL-3-Cl\% & TS707001 \\
\hline \multirow[t]{3}{*}{ L000214 } & PGCL-1-Cl\% & 053038 \\
\hline & PGCL-2-Cl\% & ARF-102-85-365 \\
\hline & PGCL-3-Cl\% & TS707001 \\
\hline \multirow[t]{3}{*}{ L000217 } & PGCL-1-Cl\% & 053038 \\
\hline & PGCL-2-Cl\% & ARF-102-85-365 \\
\hline & PGCL-3-Cl\% & TS707001 \\
\hline \multirow[t]{3}{*}{ L000223 } & PGCL-1-Cl\% & TS707001 \\
\hline & PGCL-2-Cl\% & ARF-102-85-365 \\
\hline & PGCL-3-Cl\% & 053038 \\
\hline \multirow[t]{3}{*}{ R610121 } & PGCL-1-Cl\% & $\mathrm{C} 00695$ \\
\hline & PGCL-2-Cl\% & PMAXBS \\
\hline & PGCL-3-Cl\% & ARF-102-85-223 \\
\hline \multirow[t]{3}{*}{ R610169 } & PGCL-1-Cl\% & 053038 \\
\hline & PGCL-2-Cl\% & ARF-102-85-365 \\
\hline & PGCL-3-Cl\% & |TS707001 \\
\hline
\end{tabular}

\begin{tabular}{|c|c|c|}
\hline $\begin{array}{c}3013 \\
\text { Container }\end{array}$ & $\begin{array}{l}\text { MIS Represented Item } \\
\text { Selection Order }\end{array}$ & $\begin{array}{c}\text { MIS Represented } \\
\text { Item }\end{array}$ \\
\hline \multirow[t]{3}{*}{ R610410 } & PGCL-1-Cl\% & ARF-102-85-355 \\
\hline & PGCL-2-Cl\% & $07032282 A$ \\
\hline & PGCL-3-Cl\% & $\mathrm{C} 00024 \mathrm{~A}$ \\
\hline \multirow[t]{3}{*}{ R610416 } & PGCL-1-Cl\% & 053038 \\
\hline & PGCL-2-Cl\% & ARF-102-85-365 \\
\hline & PGCL-3-Cl\% & TS707001 \\
\hline \multirow[t]{3}{*}{ R610474 } & PGCL-1-Cl\% & ARF-102-85-365 \\
\hline & PGCL-2-Cl\% & 053038 \\
\hline & PGCL-3-Cl\% & TS707001 \\
\hline \multirow[t]{3}{*}{ R610476 } & PGCL-1-CI\% & ARF-102-85-365 \\
\hline & PGCL-2-Cl\% & 053038 \\
\hline & PGCL-3-Cl\% & TS707001 \\
\hline \multirow[t]{3}{*}{ R610478 } & PGCL-1-Cl\% & ARF-102-85-365 \\
\hline & PGCL-2-Cl\% & 053038 \\
\hline & PGCL-3-Cl\% & TS707001 \\
\hline \multirow[t]{3}{*}{ R610488 } & PGCL-1-CI\% & CLLANL025 \\
\hline & PGCL-2-Cl\% & ARF-102-85-295 \\
\hline & PGCL-3-Cl\% & $\mathrm{C} 00695$ \\
\hline \multirow[t]{3}{*}{ R610492 } & PGCL-1-Cl\% & ARF-102-85-365 \\
\hline & PGCL-2-Cl\% & TS707001 \\
\hline & PGCL-3-Cl\% & 053038 \\
\hline \multirow[t]{3}{*}{ R610541 } & PGCL-1-Cl\% & 053038 \\
\hline & PGCL-2-Cl\% & ARF-102-85-365 \\
\hline & PGCL-3-Cl\% & TS707001 \\
\hline \multirow[t]{3}{*}{ R610563 } & PGCL-1-Cl\% & 053038 \\
\hline & PGCL-2-CI\% & ARF-102-85-365 \\
\hline & PGCL-3-Cl\% & TS707001 \\
\hline \multirow[t]{3}{*}{ R610573 } & PGCL-1-Cl\% & 053038 \\
\hline & PGCL-2-CI\% & ARF-102-85-365 \\
\hline & PGCL-3-Cl\% & TS707001 \\
\hline \multirow[t]{3}{*}{ R610575 } & PGCL-1-Cl\% & ARF-102-85-365 \\
\hline & PGCL-2-Cl\% & 053038 \\
\hline & PGCL-3-Cl\% & TS707001 \\
\hline \multirow[t]{3}{*}{ R610578 } & PGCL-1-Cl\% & 053038 \\
\hline & PGCL-2-Cl\% & ARF-102-85-365 \\
\hline & PGCL-3-Cl\% & TS707001 \\
\hline \multirow[t]{3}{*}{ R610585 } & PGCL-1-Cl\% & ARF-102-85-365 \\
\hline & PGCL-2-Cl\% & 053038 \\
\hline & PGCL-3-Cl\% & TS707001 \\
\hline \multirow[t]{3}{*}{ R610644 } & PGCL-1-Cl\% & 053038 \\
\hline & PGCL-2-Cl\% & ARF-102-85-365 \\
\hline & PGCL-3-Cl\% & TS707001 \\
\hline \multirow[t]{3}{*}{ R610691 } & PGCL-1-Cl\% & ARF-102-85-365 \\
\hline & PGCL-2-Cl\% & 053038 \\
\hline & PGCL-3-Cl\% & TS707001 \\
\hline \multirow[t]{3}{*}{ R610751 } & PGCL-1-Cl\% & 053038 \\
\hline & PGCL-2-Cl\% & ARF-102-85-365 \\
\hline & PGCL-3-CI\% & TS707001 \\
\hline \multirow[t]{3}{*}{ R610764 } & PGCL-1-CI\% & 053038 \\
\hline & PGCL-2-Cl\% & ARF-102-85-365 \\
\hline & PGCL-3-CI\% & TS707001 \\
\hline \multirow[t]{3}{*}{ R610765 } & PGCL-1-CI\% & C00695 \\
\hline & PGCL-2-Cl\% & CLLANL025 \\
\hline & PGCL-3-CI\% & PMAXBS \\
\hline \multirow[t]{3}{*}{ R610770 } & PGCL-1-Cl\% & 053038 \\
\hline & PGCL-2-Cl\% & ARF-102-85-365 \\
\hline & PGCL-3-Cl\% & TS707001 \\
\hline
\end{tabular}




\begin{tabular}{|c|c|c|c|c|c|}
\hline $\begin{array}{c}3013 \\
\text { Container }\end{array}$ & $\begin{array}{c}\text { MIS Represented Item } \\
\text { Selection Order }\end{array}$ & \begin{tabular}{|c|}
$\begin{array}{c}\text { MIS Represented } \\
\text { Item }\end{array}$ \\
\end{tabular} & \begin{tabular}{|c|}
3013 \\
Container \\
\end{tabular} & $\begin{array}{c}\text { MIS Represented Item } \\
\text { Selection Order }\end{array}$ & $\begin{array}{c}\text { MIS Represented } \\
\text { Item }\end{array}$ \\
\hline \multirow[t]{3}{*}{ R610776 } & PGCL-1-Cl\% & PMAXBS & \multirow[t]{3}{*}{ R610412 } & \multirow[t]{3}{*}{ PGFHi } & \multirow{3}{*}{$\begin{array}{l}07242201 A \\
64-85-12-1858 \\
\text { PuF4-1 }\end{array}$} \\
\hline & PGCL-2-Cl\% & ARF-102-85-223 & & & \\
\hline & PGCL-3-Cl\% & C00695 & & & \\
\hline \multirow[t]{3}{*}{ R610785 } & PGCL-1-Cl\% & 053038 & \multirow[t]{3}{*}{ R610434 } & \multirow[t]{3}{*}{ PGFHi } & \multirow{3}{*}{$\begin{array}{l}07242201 A \\
64-85-12-1858 \\
\text { PuF4-1 }\end{array}$} \\
\hline & PGCL-2-Cl\% & ARF-102-85-365 & & & \\
\hline & PGCL-3-Cl\% & TS707001 & & & \\
\hline \multirow[t]{3}{*}{ R610806 } & PGCL-1-Cl\% & ARF-102-85-365 & \multirow[t]{3}{*}{ R610438 } & \multirow[t]{3}{*}{ PGFHi } & \multirow{3}{*}{$\begin{array}{l}07242201 A \\
64-85-12-1858 \\
\text { PuF4-1 }\end{array}$} \\
\hline & PGCL-2-Cl\% & 053038 & & & \\
\hline & PGCL-3-Cl\% & TS707001 & & & \\
\hline \multirow[t]{3}{*}{ R610830 } & PGCL-1-Cl\% & 053038 & \multirow[t]{3}{*}{ R610451 } & \multirow[t]{3}{*}{ PGFHi } & \multirow{3}{*}{$\begin{array}{l}07242201 A \\
64-85-12-1858 \\
\text { PuF4-1 }\end{array}$} \\
\hline & PGCL-2-Cl\% & ARF-102-85-365 & & & \\
\hline & PGCL-3-Cl\% & TS707001 & & & \\
\hline \multirow[t]{3}{*}{ R610841 } & PGCL-1-Cl\% & CLLANL025 & \multirow[t]{3}{*}{ R610584 } & \multirow[t]{3}{*}{ PGFHi } & \multirow{3}{*}{$\begin{array}{l}07242201 A \\
64-85-12-1858 \\
\text { PuF4-1 }\end{array}$} \\
\hline & PGCL-2-Cl\% & ARF-102-85-295 & & & \\
\hline & PGCL-3-Cl\% & C00695 & & & \\
\hline R610930 & PGCL-1-CI\% & ARF-102-85-295 & R610595 & PGFHI & $07242201 \mathrm{~A}$ \\
\hline & PGCL-2-Cl\% & CLLANL025 & & & $64-85-12-1858$ \\
\hline & PGCL-3-Cl\% & $\mathrm{C} 00024 \mathrm{~A}$ & & & PuF4-1 \\
\hline R610940 & PGCL-1-Cl\% & TS707001 & R610599 & PGFHi & $07242201 \mathrm{~A}$ \\
\hline & PGCL-2-Cl\% & ARF-102-85-365 & & & $64-85-12-1858$ \\
\hline & PGCL-3-Cl\% & 053038 & & & PuF4-1 \\
\hline R611108 & PGCL-1-Cl\% & PMAXBS & R610611 & PGFHi & $07242201 \mathrm{~A}$ \\
\hline & PGCL-2-Cl\% & ARF-102-85-223 & & & $64-85-12-1858$ \\
\hline & PGCL-3-Cl\% & C00695 & & & PuF4-1 \\
\hline R611265 & PGCL-1-Cl\% & 053038 & R610625 & PGFHi & $07242201 \mathrm{~A}$ \\
\hline & PGCL-2-Cl\% & ARF-102-85-365 & & & $64-85-12-1858$ \\
\hline & PGCL-3-Cl\% & TS707001 & & & PuF4-1 \\
\hline$R 611370$ & PGCL-1-Cl\% & TS707001 & R610642 & PGFHi & $07242201 \mathrm{~A}$ \\
\hline & PGCL-2-Cl\% & ARF-102-85-365 & & & $64-85-12-1858$ \\
\hline & PGCL-3-Cl\% & 053038 & & & PuF4-1 \\
\hline R611398 & PGCL-1-Cl\% & TS707001 & R610668 & PGFHi & $07242201 \mathrm{~A}$ \\
\hline & PGCL-2-Cl\% & ARF-102-85-365 & & & $64-85-12-1858$ \\
\hline & PGCL-3-Cl\% & 053038 & & & PuF4-1 \\
\hline $\mathrm{H} 000790$ & PGFHi & $\begin{array}{l}07242201 A \\
64-85-12-1858 \\
\text { PuF4-1 }\end{array}$ & R610676 & PGFHi & $\begin{array}{l}07242201 A \\
64-85-12-1858 \\
\text { PuF4-1 }\end{array}$ \\
\hline $\mathrm{H} 000847$ & PGFHi & $\begin{array}{l}07242201 A \\
64-85-12-1858 \\
\text { PuF4-1 } \\
\end{array}$ & R610758 & PGFHi & $\begin{array}{l}07242201 A \\
64-85-12-1858 \\
\text { PuF4-1 }\end{array}$ \\
\hline $\mathrm{H} 003571$ & PGFHi & $\begin{array}{l}07242201 A \\
64-85-12-1858 \\
\text { PuF4-1 }\end{array}$ & R610886 & PGFHi & $\begin{array}{l}07242201 A \\
64-85-12-1858 \\
\text { PuF4-1 }\end{array}$ \\
\hline $\mathrm{H} 003651$ & PGFHi & $07242201 \mathrm{~A}$ & $\mathrm{H} 000598$ & PGFLo-1-Act $\%$ & 07161856 \\
\hline & & $64-85-12-1858$ & & PGFLo-2-F\% & $63-88-06-121$ \\
\hline & & PuF4-1 & & PGFLo-3-F\% & $07242141 \mathrm{~A}$ \\
\hline $\mathrm{H} 003713$ & PGFHI & $07242201 A$ & $\mathrm{H} 000623$ & PGFLo-1-Act $\%$ & 07161856 \\
\hline & & $64-85-12-1858$ & & PGFLo-2-F\% & $07242141 \mathrm{~A}$ \\
\hline & & PuF4-1 & & PGFLo-3-F\% & 63-88-06-121 \\
\hline L000177 & PGFHi & $07242201 \mathrm{~A}$ & $\mathrm{H} 000862$ & PGFLo-1-Act $\%$ & 07161856 \\
\hline & & $64-85-12-1858$ & & PGFLo-2-F\% & $63-88-06-121$ \\
\hline & & PuF4-1 & & PGFLo-3-F\% & $41-85-08-1379$ \\
\hline L000179 & PGFHi & $07242201 \mathrm{~A}$ & $\mathrm{H} 002716$ & PGFLo-1-Act $\%$ & 07161856 \\
\hline & & $64-85-12-1858$ & & PGFLo-2-F\% & $07242165 \mathrm{~A}$ \\
\hline & & PuF4-1 & & PGFLo-3-F\% & $64-85-12-1858$ \\
\hline R610305 & PGFHi & $07242201 \mathrm{~A}$ & $\mathrm{H} 002721$ & PGFLo-1-Act $\%$ & 07161856 \\
\hline & & $64-85-12-1858$ & & PGFLo-2-F\% & $07242165 \mathrm{~A}$ \\
\hline & & PuF4-1 & & PGFLo-3-F\% & $64-85-12-1858$ \\
\hline
\end{tabular}




\begin{tabular}{|c|c|c|}
\hline \begin{tabular}{|c|}
3013 \\
Container
\end{tabular} & $\begin{array}{l}\text { MIS Represented Item } \\
\text { Selection Order }\end{array}$ & $\begin{array}{l}\text { MIS Represented } \\
\text { Item }\end{array}$ \\
\hline \multirow[t]{3}{*}{ H003398 } & PGFLo-1-Act $\%$ & 07161856 \\
\hline & PGFLo-2-F\% & $64-85-12-1858$ \\
\hline & PGFLo-3-F\% & $41-85-08-1379$ \\
\hline \multirow[t]{3}{*}{$\mathrm{H} 003570$} & PGFLo-1-Act \% & 07161856 \\
\hline & PGFLo-2-F\% & $07242165 \mathrm{~A}$ \\
\hline & PGFLo-3-F\% & $64-85-12-1858$ \\
\hline \multirow[t]{3}{*}{ H003647 } & PGFLo-1-Act $\%$ & 07161856 \\
\hline & PGFLo-2-F\% & $07242165 \mathrm{~A}$ \\
\hline & PGFLo-3-F\% & $64-85-12-1858$ \\
\hline \multirow[t]{3}{*}{ H003654 } & PGFLo-1-Act \% & 07161856 \\
\hline & PGFLo-2-F\% & $07242165 \mathrm{~A}$ \\
\hline & PGFL0-3-F\% & $64-85-12-1858$ \\
\hline \multirow[t]{3}{*}{ H003662 } & PGFLo-1-Act $\%$ & 07161856 \\
\hline & PGFLO-2-F\% & $64-85-12-1858$ \\
\hline & PGFLo-3-F\% & $41-85-08-1379$ \\
\hline \multirow[t]{3}{*}{$\mathrm{H} 003675$} & PGFLo-1-Act $\%$ & 07161856 \\
\hline & PGFLo-2-F\% & $07242141 \mathrm{~A}$ \\
\hline & PGFLo-3-F\% & $63-88-06-121$ \\
\hline \multirow[t]{3}{*}{$\mathrm{H} 003682$} & PGFLo-1-Act $\%$ & 07161856 \\
\hline & PGFLo-2-F\% & $07242165 \mathrm{~A}$ \\
\hline & PGFLo-3-F\% & $64-85-12-1858$ \\
\hline \multirow[t]{3}{*}{$\mathrm{H} 003880$} & PGFLo-1-Act $\%$ & 07161856 \\
\hline & PGFLO-2-F\% & $07242165 \mathrm{~A}$ \\
\hline & PGFLo-3-F\% & $64-85-12-1858$ \\
\hline \multirow[t]{3}{*}{ H003975 } & PGFLo-1-Act $\%$ & 07161856 \\
\hline & PGFLo-2-F\% & $07242165 \mathrm{~A}$ \\
\hline & PGFLo-3-F\% & $64-85-12-1858$ \\
\hline \multirow[t]{3}{*}{$\mathrm{H} 004333$} & PGFLo-1-Act $\%$ & 07161856 \\
\hline & PGFLo-2-F\% & $64-85-12-1858$ \\
\hline & PGFLo-3-F\% & $07242165 A$ \\
\hline \multirow[t]{3}{*}{ L000157 } & PGFLo-1-Act $\%$ & $07242141 \mathrm{~A}$ \\
\hline & PGFLo-2-F\% & $64-85-12-1858$ \\
\hline & PGFLo-3-F\% & 07161856 \\
\hline \multirow[t]{3}{*}{$\overline{L 000158}$} & PGFLo-1-Act $\%$ & $07242141 \mathrm{~A}$ \\
\hline & PGFLo-2-F\% & $41-85-08-1379$ \\
\hline & PGFLo-3-F\% & $63-88-06-121$ \\
\hline \multirow[t]{3}{*}{ L000169 } & PGFLo-1-Act $\%$ & 07161856 \\
\hline & PGFLo-2-F\% & $64-85-12-1858$ \\
\hline & PGFLo-3-F\% & 41-85-08-1379 \\
\hline \multirow[t]{3}{*}{ L000178 } & PGFLo-1-Act $\%$ & $07242141 \mathrm{~A}$ \\
\hline & PGFLo-2-F\% & $41-85-08-1379$ \\
\hline & PGFLo-3-F\% & $63-88-06-121$ \\
\hline \multirow[t]{3}{*}{ L000180 } & PGFLo-1-Act $\%$ & $07242141 \mathrm{~A}$ \\
\hline & PGFLo-2-F\% & 64-85-12-1858 \\
\hline & PGFLo-3-F\% & 07161856 \\
\hline \multirow[t]{3}{*}{ L000182 } & PGFLo-1-Act $\%$ & $07242141 \mathrm{~A}$ \\
\hline & PGFLo-2-F\% & 07161856 \\
\hline & PGFLo-3-F\% & $07242165 \mathrm{~A}$ \\
\hline \multirow[t]{3}{*}{ L000184 } & PGFLo-1-Act $\%$ & $07242165 \mathrm{~A}$ \\
\hline & PGFLo-2-F\% & 07161856 \\
\hline & PGFLo-3-F\% & 64-85-12-1858 \\
\hline \multirow[t]{3}{*}{ L000185 } & PGFLo-1-Act $\%$ & 63-88-06-121 \\
\hline & PGFLo-2-F\% & 07161856 \\
\hline & PGFLo-3-F\% & 64-85-12-1858 \\
\hline \multirow[t]{3}{*}{ L000186 } & PGFLo-1-Act $\%$ & $07242141 \mathrm{~A}$ \\
\hline & PGFLO-2-F\% & $63-88-06-121$ \\
\hline & PGFLo-3-F\% & $41-85-08-1379$ \\
\hline
\end{tabular}

\begin{tabular}{|c|c|c|}
\hline $\begin{array}{c}3013 \\
\text { Container }\end{array}$ & $\begin{array}{l}\text { MIS Represented Item } \\
\text { Selection Order }\end{array}$ & $\begin{array}{c}\text { MIS Represented } \\
\text { Item }\end{array}$ \\
\hline \multirow[t]{3}{*}{ L000220 } & PGFLo-1-Act $\%$ & 07161856 \\
\hline & PGFLo-2-F\% & $07242165 \mathrm{~A}$ \\
\hline & PGFLo-3-F\% & $64-85-12-1858$ \\
\hline \multirow[t]{3}{*}{ L000227 } & PGFLo-1-Act $\%$ & $07242141 \mathrm{~A}$ \\
\hline & PGFLo-2-F\% & $07242165 \mathrm{~A}$ \\
\hline & PGFLo-3-F\% & 07161856 \\
\hline \multirow[t]{3}{*}{ L000228 } & PGFLo-1-Act $\%$ & $07242141 \mathrm{~A}$ \\
\hline & PGFLo-2-F\% & 07161856 \\
\hline & PGFLo-3-F\% & $64-85-12-1858$ \\
\hline \multirow[t]{3}{*}{ R601284 } & PGFLo-1-Act $\%$ & 07161856 \\
\hline & PGFLo-2-F\% & $64-85-12-1858$ \\
\hline & PGFLo-3-F\% & $07242165 \mathrm{~A}$ \\
\hline \multirow[t]{3}{*}{$\mathrm{R} 601456$} & PGFLo-1-Act $\%$ & 07161856 \\
\hline & PGFLo-2-F\% & $07242165 A$ \\
\hline & PGFLo-3-F\% & 64-85-12-1858 \\
\hline \multirow[t]{3}{*}{ R610263 } & PGFLo-1-Act $\%$ & 07161856 \\
\hline & PGFLo-2-F\% & $41-85-08-1379$ \\
\hline & PGFLo-3-F $\%$ & $64-85-12-1858$ \\
\hline \multirow[t]{3}{*}{ R610319 } & PGFLo-1-Act $\%$ & $07242141 \mathrm{~A}$ \\
\hline & PGFLo-2-F\% & $63-88-06-121$ \\
\hline & PGFLo-3-F\% & $41-85-08-1379$ \\
\hline \multirow[t]{3}{*}{ R610428 } & PGFLo-1-Act $\%$ & 07161856 \\
\hline & PGFLo-2-F\% & 63-88-06-121 \\
\hline & PGFLo-3-F\% & $07242141 \mathrm{~A}$ \\
\hline \multirow[t]{3}{*}{ R610430 } & PGFLo-1-Act $\%$ & 07161856 \\
\hline & PGFLo-2-F\% & $41-85-08-1379$ \\
\hline & PGFLo-3-F\% & $63-88-06-121$ \\
\hline \multirow[t]{3}{*}{ R610432 } & PGFLo-1-Act $\%$ & $07242141 \mathrm{~A}$ \\
\hline & PGFLo-2-F\% & $63-88-06-121$ \\
\hline & PGFLo-3-F\% & $41-85-08-1379$ \\
\hline \multirow[t]{3}{*}{ R610475 } & PGFLo-1-Act $\%$ & 07161856 \\
\hline & PGFLo-2-F\% & $41-85-08-1379$ \\
\hline & PGFLo-3-F\% & $64-85-12-1858$ \\
\hline \multirow[t]{3}{*}{ R610548 } & PGFLo-1-Act $\%$ & $07242141 \mathrm{~A}$ \\
\hline & PGFLo-2-F\% & $41-85-08-1379$ \\
\hline & PGFLo-3-F\% & $63-88-06-121$ \\
\hline \multirow[t]{3}{*}{ R610552 } & PGFLo-1-Act $\%$ & 07161856 \\
\hline & PGFLo-2-F\% & $07242141 \mathrm{~A}$ \\
\hline & PGFLo-3-F\% & $63-88-06-121$ \\
\hline \multirow[t]{3}{*}{ R610564 } & PGFLo-1-Act $\%$ & 07161856 \\
\hline & PGFLo-2-F\% & $07242141 \mathrm{~A}$ \\
\hline & PGFLo-3-F\% & $63-88-06-121$ \\
\hline \multirow[t]{3}{*}{ R610576 } & PGFLo-1-Act $\%$ & 07161856 \\
\hline & PGFLo-2-F\% & $07242165 A$ \\
\hline & PGFL0-3-F\% & $64-85-12-1858$ \\
\hline \multirow[t]{3}{*}{ R610580 } & PGFLo-1-Act $\%$ & 07161856 \\
\hline & PGFLo-2-F\% & $63-88-06-121$ \\
\hline & PGFLo-3-F\% & $07242141 \mathrm{~A}$ \\
\hline \multirow[t]{3}{*}{ R610582 } & PGFLo-1-Act $\%$ & 07161856 \\
\hline & PGFLo-2-F\% & $07242165 A$ \\
\hline & PGFLo-3-F\% & $64-85-12-1858$ \\
\hline \multirow[t]{3}{*}{ R610622 } & PGFL0-1-Act $\%$ & 07161856 \\
\hline & PGFLo-2-F\% & $07242141 \mathrm{~A}$ \\
\hline & PGFLo-3-F\% & $63-88-06-121$ \\
\hline \multirow[t]{3}{*}{ R610685 } & PGFLo-1-Act $\%$ & 07161856 \\
\hline & PGFLo-2-F\% & $07242141 \mathrm{~A}$ \\
\hline & PGFLo-3-F\% & $63-88-06-121$ \\
\hline
\end{tabular}




\begin{tabular}{|c|c|c|}
\hline $\begin{array}{c}3013 \\
\text { Container }\end{array}$ & $\begin{array}{l}\text { MIS Represented Item } \\
\text { Selection Order }\end{array}$ & $\begin{array}{l}\text { MIS Represented } \\
\text { Item }\end{array}$ \\
\hline \multirow[t]{3}{*}{ R610891 } & PGFLo-1-Act\% & 07161856 \\
\hline & PGFLo-2-F\% & $07242165 \mathrm{~A}$ \\
\hline & PGFLo-3-F\% & $64-85-12-1858$ \\
\hline \multirow[t]{3}{*}{$\mathrm{H} 003951$} & PGFLo-1-Act $\%$ & 07161856 \\
\hline & PGFLo-2-F\% & $07242165 A$ \\
\hline & PGFLo-3-F\% & $64-85-12-1858$ \\
\hline \multirow[t]{3}{*}{$\mathrm{H} 000806$} & PGMisc-NoU-1-Act $\%$ & PPSL-365 \\
\hline & PGMisc-NoU-2a-Be & ARF-102-85-114-1 \\
\hline & PGMisc-NoU-WG & MT1490 \\
\hline \multirow[t]{3}{*}{$\mathrm{H} 000823$} & PGMisc-NoU-1-Act $\%$ & PPSL-365 \\
\hline & PGMisc-NoU-2a-Be & ARF-102-85-114-1 \\
\hline & PGMisc-NoU-WG & MT1490 \\
\hline \multirow[t]{3}{*}{ H000921 } & PGMisc+U-1-U\% & PSU-84-06-05 \\
\hline & PGMisc+U-2-Act\% & CAN92 \\
\hline & PGMisc+U-3-WG & ARF-102-85-114-1 \\
\hline \multirow[t]{3}{*}{$\mathrm{H} 002129$} & PGMisc-NoU-1-Act $\%$ & BLO-39-11-14-004 \\
\hline & PGMisc-NoU-2b-NoBe-Act\% & MT1490 \\
\hline & PGMisc-NoU-NWG & PBO-47-09-012-023 \\
\hline \multirow[t]{3}{*}{$\mathrm{H} 002173$} & PGMisc+U-1-U\% & CAN92 \\
\hline & PGMisc+U-2-Act\% & 669194 \\
\hline & PGMisc+U-3-NWG & BLO-39-11-14-004 \\
\hline \multirow[t]{3}{*}{$\mathrm{H} 002300$} & PGMisc+U-1-U\% & 5501407 \\
\hline & PGMisc+U-2-Act\% & PSU-84-06-05 \\
\hline & PGMisc+U-3-NWG & BLO-39-11-14-004 \\
\hline \multirow[t]{3}{*}{$\mathrm{H} 002366$} & PGMisc+U-1-U\% & 5501407 \\
\hline & PGMisc+U-2-Act\% & PSU-84-06-05 \\
\hline & PGMisc+U-3-NWG & PPSL-365 \\
\hline \multirow[t]{3}{*}{$\mathrm{H} 002373$} & PGMisc+U-1-U\% & 5501407 \\
\hline & PGMisc+U-2-Act\% & 669194 \\
\hline & PGMisc+U-3-WG & PPSL-365 \\
\hline \multirow[t]{3}{*}{$\mathrm{H} 002389$} & PGMisc+U-1-U\% & 5501407 \\
\hline & PGMisc+U-2-Act\% & CAN92 \\
\hline & PGMisc+U-3-NWG & PSU-84-06-05 \\
\hline \multirow[t]{3}{*}{ H002400 } & PGMisc+U-1-U\% & 669194 \\
\hline & PGMisc+U-2-Act\% & 5501407 \\
\hline & PGMisc+U-3-NWG & BLO-39-11-14-004 \\
\hline \multirow[t]{3}{*}{$\mathrm{H} 002401$} & PGMisc+U-1-U\% & CAN92 \\
\hline & PGMisc+U-2-Act\% & 669194 \\
\hline & PGMisc+U-3-WG & ARF-102-85-114-1 \\
\hline \multirow[t]{3}{*}{$\mathrm{H} 002409$} & PGMisc+U-1-U\% & 5501407 \\
\hline & PGMisc+U-2-Act $\%$ & PSU-84-06-05 \\
\hline & PGMisc+U-3-NWG & PPSL-365 \\
\hline \multirow[t]{3}{*}{$\mathrm{H} 002412$} & PGMisc+U-1-U\% & 669194 \\
\hline & PGMisc+U-2-Act $\%$ & CAN92 \\
\hline & PGMisc+U-3-NWG & BLO-39-11-14-004 \\
\hline \multirow[t]{3}{*}{$\mathrm{H} 002489$} & PGMisc+U-1-U\% & CAN92 \\
\hline & PGMisc+U-2-Act\% & 669194 \\
\hline & PGMisc+U-3-NWG & BLO-39-11-14-004 \\
\hline \multirow[t]{3}{*}{$\mathrm{H} 002504$} & PGMisc+U-1-U\% & CAN92 \\
\hline & PGMisc+U-2-Act $\%$ & 669194 \\
\hline & PGMisc+U-3-NWG & BLO-39-11-14-004 \\
\hline \multirow[t]{3}{*}{ H002505 } & PGMisc+U-1-U\% & 5501407 \\
\hline & PGMisc+U-2-Act $\%$ & CAN92 \\
\hline & PGMisc+U-3-NWG & BLO-39-11-14-004 \\
\hline \multirow[t]{3}{*}{ H002537 } & PGMisc+U-1-U\% & CAN92 \\
\hline & PGMisc+U-2-Act\% & 669194 \\
\hline & PGMisc+U-3-NWG & BLO-39-11-14-004 \\
\hline
\end{tabular}

\begin{tabular}{|c|c|c|}
\hline $\begin{array}{c}3013 \\
\text { Container }\end{array}$ & $\begin{array}{l}\text { MIS Represented Item } \\
\text { Selection Order }\end{array}$ & $\begin{array}{l}\text { MIS Represented } \\
\text { Item } \\
\end{array}$ \\
\hline \multirow[t]{3}{*}{$\mathrm{H} 002540$} & PGMisc+U-1-U\% & CAN92 \\
\hline & PGMisc+U-2-Act $\%$ & 669194 \\
\hline & PGMisc+U-3-NWG & BLO-39-11-14-004 \\
\hline \multirow[t]{3}{*}{$\mathrm{H} 002541$} & PGMisc+U-1-U\% & CAN92 \\
\hline & PGMisc+U-2-Act $\%$ & 669194 \\
\hline & PGMisc+U-3-NWG & BLO-39-11-14-004 \\
\hline \multirow[t]{3}{*}{$\mathrm{H} 002561$} & PGMisc+U-1-U\% & CAN92 \\
\hline & PGMisc+U-2-Act $\%$ & 669194 \\
\hline & PGMisc+U-3-NWG & BLO-39-11-14-004 \\
\hline \multirow[t]{3}{*}{$\mathrm{H} 002627$} & PGMisc-NoU-1-Act \% & PPSL-365 \\
\hline & PGMisc-NoU-2b-NoBe-Act\% & BLO-39-11-14-004 \\
\hline & PGMisc-NoU-NWG & PBO-47-09-012-023 \\
\hline \multirow[t]{3}{*}{$\mathrm{H} 002632$} & PGMisc-NoU-1-Act\% & PPSL-365 \\
\hline & PGMisc-NoU-2b-NoBe-Act\% & BLO-39-11-14-004 \\
\hline & PGMisc-NoU-NWG & $\mathrm{PBO}-47-09-012-023$ \\
\hline \multirow[t]{3}{*}{$\mathrm{H} 002676$} & PGMisc-NoU-1-Act $\%$ & BLO-39-11-14-004 \\
\hline & PGMisc-NoU-2b-NoBe-Act $\%$ & MT1490 \\
\hline & PGMisc-NoU-NWG & PBO-47-09-012-023 \\
\hline \multirow[t]{3}{*}{$\mathrm{H} 002683$} & PGMisC+U-1-U\% & 5501407 \\
\hline & PGMisc+U-2-Act $\%$ & PSU-84-06-05 \\
\hline & PGMisc+U-3-NWG & $\mathrm{PBO}-47-09-012-023$ \\
\hline \multirow[t]{3}{*}{$\mathrm{H} 002710$} & PGMisc+U-1-U\% & PSU-84-06-05 \\
\hline & PGMisc+U-2-Act $\%$ & 5501407 \\
\hline & PGMisc+U-3-NWG & $\mathrm{PBO}-47-09-012-023$ \\
\hline \multirow[t]{3}{*}{$\mathrm{H} 002718$} & PGMisc+U-1-U\% & CAN92 \\
\hline & PGMisC+U-2-Act $\%$ & 669194 \\
\hline & PGMisc+U-3-NWG & BLO-39-11-14-004 \\
\hline \multirow[t]{3}{*}{$\mathrm{H} 002733$} & PGMisc-NoU-1-Act $\%$ & PPSL-365 \\
\hline & PGMisc-NoU-2b-NoBe-Act\% & BLO-39-11-14-004 \\
\hline & PGMisc-NoU-NWG & $\mathrm{PBO}-47-09-012-023$ \\
\hline \multirow[t]{3}{*}{$\mathrm{H} 003347$} & PGMisc+U-1-U\% & PSU-84-06-05 \\
\hline & PGMisc+U-2-Act $\%$ & 5501407 \\
\hline & PGMisc+U-3-NWG & PPSL-365 \\
\hline \multirow[t]{3}{*}{ H003359 } & PGMisc-NoU-1-Act $\%$ & $66-01-01-439$ \\
\hline & PGMisc-NoU-2b-NoBe-Act $\%$ & PPSL-365 \\
\hline & PGMisc-NoU-NWG & PBO-47-09-012-023 \\
\hline \multirow[t]{3}{*}{$\mathrm{H} 003386$} & PGMisc+U-1-U\% & PSU-84-06-05 \\
\hline & PGMisc+U-2-Act\% & 669194 \\
\hline & PGMisC+U-3-NWG & PBO-47-09-012-023 \\
\hline \multirow[t]{3}{*}{ H003388 } & PGMisc+U-1-U\% & 669194 \\
\hline & PGMisc+U-2-Act\% & CAN92 \\
\hline & PGMisc+U-3-NWG & PBO-47-09-012-023 \\
\hline \multirow[t]{3}{*}{$\mathrm{H} 003446$} & PGMisc+U-1-U\% & PSU-84-06-05 \\
\hline & PGMisc+U-2-Act \% & 5501407 \\
\hline & PGMisc+U-3-NWG & PPSL-365 \\
\hline \multirow[t]{3}{*}{$\mathrm{H} 003530$} & PGMisc-NoU-1-Act $\%$ & 66-01-01-439 \\
\hline & PGMisc-NoU-2b-NoBe-Act\% & PPSL-365 \\
\hline & PGMisc-NoU-NWG & BLO-39-11-14-004 \\
\hline \multirow[t]{3}{*}{ H003562 } & PGMisc-NoU-1-Act \% & PPSL-365 \\
\hline & PGMisc-NoU-2b-NoBe-Act\% & BLO-39-11-14-004 \\
\hline & PGMisc-NoU-NWG & PBO-47-09-012-023 \\
\hline \multirow[t]{3}{*}{$\mathrm{H} 003601$} & PGMisc-NoU-1-Act $\%$ & ARF-102-85-114-1 \\
\hline & PGMisc-NoU-2b-NoBe-Act $\%$ & $\mathrm{PBO}-47-09-012-023$ \\
\hline & PGMisc-NoU-NWG & BLO-39-11-14-004 \\
\hline \multirow[t]{3}{*}{$\mathrm{H} 003628$} & PGMisc-NoU-1-Act $\%$ & BLO-39-11-14-004 \\
\hline & PGMisc-NoU-2b-NoBe-Act $\%$ & MT1490 \\
\hline & PGMisc-NoU-NWG & PBO-47-09-012-023 \\
\hline
\end{tabular}




\begin{tabular}{|c|c|c|}
\hline $\begin{array}{c}3013 \\
\text { Container }\end{array}$ & \begin{tabular}{|c|}
$\begin{array}{c}\text { MIS Represented Item } \\
\text { Selection Order }\end{array}$ \\
\end{tabular} & $\begin{array}{c}\begin{array}{c}\text { MIS Represented } \\
\text { Item }\end{array} \\
\end{array}$ \\
\hline \multirow[t]{3}{*}{$\mathrm{H} 003633$} & PGMisc-NoU-1-Act\% & MT1490 \\
\hline & PGMisc-NoU-2b-NoBe-Act\% & BLO-39-11-14-004 \\
\hline & PGMisc-NoU-NWG & PBO-47-09-012-023 \\
\hline \multirow[t]{3}{*}{$\mathrm{H} 003641$} & PGMisc+U-1-U\% & PSU-84-06-05 \\
\hline & PGMisc+U-2-Act\% & 669194 \\
\hline & PGMisc+U-3-NWG & PBO-47-09-012-023 \\
\hline \multirow[t]{3}{*}{$\mathrm{H} 003684$} & PGMisc-NoU-1-Act \% & $66-01-01-439$ \\
\hline & PGMisc-NoU-2b-NoBe-Act $\%$ & PPSL-365 \\
\hline & PGMisc-NoU-NWG & BLO-39-11-14-004 \\
\hline \multirow[t]{3}{*}{$\mathrm{H} 003723$} & PGMisc-NoU-1-Act $\%$ & BLO-39-11-14-004 \\
\hline & PGMisc-NoU-2b-NoBe-Act\% & MT1490 \\
\hline & PGMisc-NoU-NWG & PBO-47-09-012-023 \\
\hline \multirow[t]{3}{*}{$\mathrm{H} 003733$} & PGMisc+U-1-U\% & PSU-84-06-05 \\
\hline & PGMisc+U-2-Act\% & 5501407 \\
\hline & PGMisc+U-3-NWG & PBO-47-09-012-023 \\
\hline \multirow[t]{3}{*}{$\mathrm{H} 003787$} & PGMisc+U-1-U\% & PSU-84-06-05 \\
\hline & PGMisc+U-2-Act\% & 669194 \\
\hline & PGMisc+U-3-NWG & PPSL-365 \\
\hline \multirow[t]{3}{*}{$\mathrm{H} 003845$} & PGMisc+U-1-U\% & PSU-84-06-05 \\
\hline & PGMisc+U-2-Act\% & 5501407 \\
\hline & PGMisc+U-3-WG & $66-01-01-439$ \\
\hline \multirow[t]{3}{*}{$\mathrm{H} 003903$} & PGMisc+U-1-U\% & 5501407 \\
\hline & PGMisc+U-2-Act\% & PSU-84-06-05 \\
\hline & PGMisc+U-3-NWG & PPSL-365 \\
\hline \multirow[t]{3}{*}{$\mathrm{H} 003967$} & PGMisc-NoU-1-Act $\%$ & $66-01-01-439$ \\
\hline & PGMisc-NoU-2b-NoBe-Act\% & PPSL-365 \\
\hline & PGMisc-NoU-NWG & PBO-47-09-012-023 \\
\hline \multirow[t]{3}{*}{$\mathrm{H} 004409$} & PGMisc+U-1-U\% & CAN92 \\
\hline & PGMisc+U-2-Act\% & 5501407 \\
\hline & PGMisc+U-3-NWG & PSU-84-06-05 \\
\hline \multirow[t]{3}{*}{ L000058 } & PGMisc+U-1-U\% & CAN92 \\
\hline & PGMisc+U-2-Act\% & 669194 \\
\hline & PGMisc+U-3-Be-WG & ARF-102-85-114-1 \\
\hline \multirow[t]{3}{*}{ L000071 } & PGMisc+U-1-U\% & CAN92 \\
\hline & PGMisc+U-2-Act\% & 669194 \\
\hline & PGMisc+U-3-WG & PPSL-365 \\
\hline \multirow[t]{3}{*}{ L000072 } & PGMisc+U-1-U\% & 5501407 \\
\hline & PGMisc+U-2-Act\% & PSU-84-06-05 \\
\hline & PGMisc+U-3-WG & $66-01-01-439$ \\
\hline \multirow[t]{3}{*}{ L000073 } & PGMisc-NoU-1-Act $\%$ & BLO-39-11-14-004 \\
\hline & PGMisc-NoU-2b-NoBe-Act $\%$ & MT1490 \\
\hline & PGMisc-NoU-WG & PPSL-365 \\
\hline \multirow[t]{3}{*}{ L000074 } & PGMisc+U-1-U\% & 669194 \\
\hline & PGMisc+U-2-Act\% & CAN92 \\
\hline & PGMisc+U-3-WG & MT1490 \\
\hline \multirow[t]{3}{*}{ L000075 } & PGMisc+U-1-U\% & CAN92 \\
\hline & PGMisc+U-2-Act\% & PSU-84-06-05 \\
\hline & PGMisc+U-3-Be-WG & ARF-102-85-114-1 \\
\hline \multirow[t]{3}{*}{ L000077 } & PGMisc+U-1-U\% & 5501407 \\
\hline & PGMisc+U-2-Act\% & PSU-84-06-05 \\
\hline & PGMisc+U-3-WG & 669194 \\
\hline \multirow[t]{3}{*}{ L000151 } & PGMisC+U-1-U\% & PSU-84-06-05 \\
\hline & PGMisc+U-2-Act\% & 5501407 \\
\hline & PGMisc+U-3-WG & 669194 \\
\hline \multirow[t]{3}{*}{ L000152 } & PGMisc-NoU-1-Act\% & $66-01-01-439$ \\
\hline & PGMisc-NoU-2a-Be & ARF-102-85-114-1 \\
\hline & PGMisc-NoU-NWG & PBO-47-09-012-023 \\
\hline
\end{tabular}

\begin{tabular}{|c|c|c|}
\hline $\begin{array}{c}3013 \\
\text { Container }\end{array}$ & $\begin{array}{l}\text { MIS Represented Item } \\
\text { Selection Order }\end{array}$ & $\begin{array}{l}\text { MIS Represented } \\
\text { Item }\end{array}$ \\
\hline \multirow[t]{3}{*}{ L000153 } & PGMisc-NoU-1-Act\% & $66-01-01-439$ \\
\hline & PGMisc-NoU-2a-Be & ARF-102-85-114-1 \\
\hline & PGMisc-NoU-WG & PPSL-365 \\
\hline \multirow[t]{3}{*}{ L000156 } & PGMisc+U-1-U\% & CAN92 \\
\hline & PGMisc+U-2-Act $\%$ & 669194 \\
\hline & PGMisc+U-3-Be-WG & ARF-102-85-114-1 \\
\hline \multirow[t]{3}{*}{ L000159 } & PGMisc+U-1-U\% & CAN92 \\
\hline & PGMisc+U-2-Act\% & 5501407 \\
\hline & PGMisc+U-3-Be-WG & ARF-102-85-114-1 \\
\hline \multirow[t]{3}{*}{ L000162 } & PGMisc $+U-1-U \%$ & 5501407 \\
\hline & PGMisc+U-2-Act $\%$ & PSU-84-06-05 \\
\hline & PGMisc+U-3-Be-WG & CAN92 \\
\hline \multirow[t]{3}{*}{ L000163 } & PGMisc-NoU-1-Act $\%$ & PPSL-365 \\
\hline & PGMisc-NoU-2b-NoBe-Act\% & BLO-39-11-14-004 \\
\hline & PGMisc-NoU-NWG & PBO-47-09-012-023 \\
\hline \multirow[t]{3}{*}{ L000164 } & PGMisc+U-1-U\% & CAN92 \\
\hline & PGMisc+U-2-Act $\%$ & 5501407 \\
\hline & PGMisc+U-3-Be-WG & ARF-102-85-114-1 \\
\hline \multirow[t]{3}{*}{ L000165 } & PGMisc-NoU-1-Act $\%$ & $66-01-01-439$ \\
\hline & PGMisc-NoU-2a-Be & ARF-102-85-114-1 \\
\hline & PGMisc-NoU-WG & PPSL-365 \\
\hline \multirow[t]{3}{*}{ L000166 } & PGMisc+U-1-U\% & CAN92 \\
\hline & PGMisc+U-2-Act $\%$ & 5501407 \\
\hline & PGMisc+U-3-WG & $66-01-01-439$ \\
\hline \multirow[t]{3}{*}{ L000170 } & PGMisc+U-1-U\% & 5501407 \\
\hline & PGMisc+U-2-Act $\%$ & PSU-84-06-05 \\
\hline & PGMisc+U-3-WG & $66-01-01-439$ \\
\hline \multirow[t]{3}{*}{ L000171 } & PGMisc-NoU-1-Act $\%$ & $66-00-11-355$ \\
\hline & PGMisc-NoU-2b-NoBe-Act\% & $66-01-01-439$ \\
\hline & PGMisc-NoU-NWG & PBO-47-09-012-023 \\
\hline \multirow[t]{3}{*}{ L000172 } & PGMisc $+U-1-U \%$ & CAN92 \\
\hline & PGMisc+U-2-Act $\%$ & 5501407 \\
\hline & PGMisc+U-3-NWG & PBO-47-09-012-023 \\
\hline \multirow[t]{3}{*}{ L000173 } & PGMisc-NoU-1-Act $\%$ & $66-01-01-439$ \\
\hline & PGMisc-NoU-2b-NoBe-Act $\%$ & PPSL-365 \\
\hline & PGMisc-NoU-WG & MT1490 \\
\hline \multirow[t]{3}{*}{ L000174 } & PGMisc-NoU-1-Act $\%$ & $66-01-01-439$ \\
\hline & PGMisc-NoU-2a-Be & ARF-102-85-114-1 \\
\hline & PGMisc-NoU-WG & PPSL-365 \\
\hline \multirow[t]{3}{*}{ L000175 } & PGMisc+U-1-U\% & CAN92 \\
\hline & PGMisc+U-2-Act $\%$ & 5501407 \\
\hline & PGMisc+U-3-Be-WG & ARF-102-85-114-1 \\
\hline \multirow[t]{3}{*}{ L000176 } & PGMisc-NoU-1-Act $\%$ & $66-01-01-439$ \\
\hline & PGMisc-NoU-2a-Be & ARF-102-85-114-1 \\
\hline & PGMisc-NoU-WG & PPSL-365 \\
\hline \multirow[t]{3}{*}{ L000191 } & PGMisc+U-1-U\% & CAN92 \\
\hline & PGMisc+U-2-Act $\%$ & 5501407 \\
\hline & PGMisc+U-3-NWG & PSU-84-06-05 \\
\hline \multirow[t]{3}{*}{ L000194 } & PGMisc+U-1-U\% & CAN92 \\
\hline & PGMisc+U-2-Act $\%$ & 5501407 \\
\hline & PGMisc+U-3-Be-WG & ARF-102-85-114-1 \\
\hline \multirow[t]{3}{*}{ L000195 } & PGMisc+U-1-U\% & CAN92 \\
\hline & PGMisc+U-2-Act $\%$ & 5501407 \\
\hline & PGMisc+U-3-Be-WG & ARF-102-85-114-1 \\
\hline \multirow[t]{3}{*}{ L000196 } & PGMisc-NoU-1-Act\% & $66-01-01-439$ \\
\hline & PGMisc-NoU-2a-Be & ARF-102-85-114-1 \\
\hline & PGMisc-NoU-WG & PPSL-365 \\
\hline
\end{tabular}




\begin{tabular}{|c|c|c|c|c|c|}
\hline $\begin{array}{c}3013 \\
\text { Container }\end{array}$ & $\begin{array}{l}\text { MIS Represented Item } \\
\text { Selection Order }\end{array}$ & $\begin{array}{l}\text { MIS Represented } \\
\text { Item }\end{array}$ & $\begin{array}{c}3013 \\
\text { Container }\end{array}$ & $\begin{array}{l}\text { MIS Represented Item } \\
\text { Selection Order }\end{array}$ & $\begin{array}{c}\text { MIS Represented } \\
\text { Item }\end{array}$ \\
\hline \multirow[t]{3}{*}{ L000199 } & PGMisc+U-1-U\% & CAN92 & \multirow[t]{3}{*}{ R610286 } & PGMisc-NoU-1-Act\% & PPSL-365 \\
\hline & PGMisc+U-2-Act\% & 5501407 & & PGMisc-NoU-2a-Be & ARF-102-85-114-1 \\
\hline & PGMisc+U-3-NWG & PPSL-365 & & PGMisc-NoU-WG & MT1490 \\
\hline \multirow[t]{3}{*}{ L000202 } & PGMisc+U-1-U\% & CAN92 & \multirow[t]{3}{*}{$R 610307$} & PGMisc+U-1-U\% & 5501407 \\
\hline & PGMisc+U-2-Act \% & 5501407 & & PGMisc+U-2-Act $\%$ & PSU-84-06-05 \\
\hline & PGMisc+U-3-NWG & PSU-84-06-05 & & PGMisc+U-3-Be-WG & CAN92 \\
\hline \multirow[t]{3}{*}{ L000203 } & PGMisc+U-1-U\% & CAN92 & \multirow[t]{3}{*}{ R610315 } & PGMisc-NoU-1-Act $\%$ & PPSL-365 \\
\hline & PGMisc+U-2-Act $\%$ & 5501407 & & PGMisc-NoU-2a-Be & ARF-102-85-114-1 \\
\hline & PGMisc+U-3-Be-WG & ARF-102-85-114-1 & & PGMisc-NoU-WG & MT1490 \\
\hline \multirow[t]{3}{*}{ L000205 } & PGMisc+U-1-U\% & CAN92 & \multirow[t]{3}{*}{ R610394 } & PGMisc+U-1-U\% & CAN92 \\
\hline & PGMisc+U-2-Act\% & 5501407 & & PGMisc+U-2-Act\% & 5501407 \\
\hline & PGMisc+U-3-WG & $66-01-01-439$ & & PGMisc+U-3-Be-WG & ARF-102-85-114-1 \\
\hline \multirow[t]{3}{*}{ L000206 } & PGMisc+U-1-U\% & 5501407 & \multirow[t]{3}{*}{ R610402 } & PGMisc-NoU-1-Act $\%$ & $66-01-01-439$ \\
\hline & PGMisc+U-2-Act $\%$ & PSU-84-06-05 & & PGMisc-NoU-2a-Be & ARF-102-85-114-1 \\
\hline & PGMisc+U-3-NWG & PPSL-365 & & PGMisc-NoU-WG & PPSL-365 \\
\hline \multirow[t]{3}{*}{ L000207 } & PGMisc+U-1-U\% & CAN92 & \multirow[t]{3}{*}{ R610470 } & PGMisc+U-1-U\% & CAN92 \\
\hline & PGMisc+U-2-Act\% & 5501407 & & PGMisc+U-2-Act $\%$ & 5501407 \\
\hline & PGMisc+U-3-Be-WG & ARF-102-85-114-1 & & PGMisc+U-3-Be-WG & ARF-102-85-114-1 \\
\hline \multirow[t]{3}{*}{ L000209 } & PGMisc+U-1-U\% & CAN92 & \multirow[t]{3}{*}{ R610473 } & PGMisc-NoU-1-Act\% & $66-00-11-355$ \\
\hline & PGMisc+U-2-Act $\%$ & 5501407 & & PGMisc-NoU-2a-Be & ARF-102-85-114-1 \\
\hline & PGMisc+U-3-Be-WG & ARF-102-85-114-1 & & PGMisc-NoU-WG & $66-01-01-439$ \\
\hline \multirow[t]{3}{*}{ L000211 } & PGMisc-NoU-1-Act $\%$ & $66-00-11-355$ & \multirow[t]{3}{*}{ R610482 } & PGMisc+U-1-U\% & CAN92 \\
\hline & PGMisc-NoU-2a-Be & ARF-102-85-114-1 & & PGMisc+U-2-Act $\%$ & 5501407 \\
\hline & PGMisc-NoU-NWG & PBO-47-09-012-023 & & PGMisc+U-3-Be-WG & ARF-102-85-114-1 \\
\hline \multirow[t]{3}{*}{ L000212 } & PGMisc-NoU-1-Act $\%$ & PPSL-365 & R610497 & PGMisc+U-1-U\% & 5501407 \\
\hline & PGMisc-NoU-2b-NoBe-Act $\%$ & BLO-39-11-14-004 & & PGMisc+U-2-Act $\%$ & CAN92 \\
\hline & PGMisc-NoU-NWG & PBO-47-09-012-023 & & PGMisc+U-3-Be-WG & ARF-102-85-114-1 \\
\hline L000219 & PGMisc-NoU-1-Act\% & $66-00-11-355$ & R610515 & PGMisc-NoU-1-Act $\%$ & PPSL-365 \\
\hline & PGMisc-NoU-2a-Be & ARF-102-85-114-1 & & PGMisc-NoU-2a-Be & ARF-102-85-114-1 \\
\hline & PGMisc-NoU-NWG & PBO-47-09-012-023 & & PGMisc-NoU-WG & MT1490 \\
\hline L000221 & PGMisc-NoU-1-Act\% & $66-01-01-439$ & R610526 & PGMisc-NoU-1-Act \% & $66-01-01-439$ \\
\hline & PGMisc-NoU-2a-Be & ARF-102-85-114-1 & & PGMisc-NoU-2a-Be & ARF-102-85-114-1 \\
\hline & PGMisC-NoU-NWG & PBO-47-09-012-023 & & PGMisc-NoU-WG & PPSL-365 \\
\hline L000224 & PGMisc+U-1-U\% & CAN92 & R610528 & PGMisc-NoU-1-Act\% & 66-01-01-439 \\
\hline & PGMisC+U-2-Act $\%$ & 5501407 & & PGMisc-NoU-2a-Be & ARF-102-85-114-1 \\
\hline & PGMisc+U-3-NWG & PSU-84-06-05 & & PGMisc-NoU-WG & PPSL-365 \\
\hline L000225 & PGMisc-NoU-1-Act \% & PPSL-365 & R610544 & PGMisc-NoU-1-Act\% & PPSL-365 \\
\hline & PGMisc-NoU-2a-Be & ARF-102-85-114-1 & & PGMisc-NoU-2b-NoBe-Act\% & BLO-39-11-14-004 \\
\hline & PGMisc-NoU-WG & $66-01-01-439$ & & PGMisc-NoU-WG & MT1490 \\
\hline L000226 & PGMisc-NoU-1-Act \% & $66-00-11-355$ & R610553 & PGMisc-NoU-1-Act \% & $66-01-01-439$ \\
\hline & PGMisc-NoU-2a-Be & ARF-102-85-114-1 & & PGMisc-NoU-2a-Be & ARF-102-85-114-1 \\
\hline & PGMisc-NoU-NWG & PBO-47-09-012-023 & & PGMisc-NoU-WG & PPSL-365 \\
\hline $\mathrm{R} 600760$ & PGMisc-NoU-1-Act $\%$ & ARF-102-85-114-1 & R610559 & PGMisc+U-1-U\% & 5501407 \\
\hline & PGMisc-NoU-2b-NoBe-Act $\%$ & MT1490 & & PGMisc+U-2-Act\% & PSU-84-06-05 \\
\hline & PGMisc-NoU-WG & PPSL-365 & & PGMisc+U-3-Be-WG & CAN92 \\
\hline $\mathrm{R} 601365$ & PGMisc-NoU-1-Act $\%$ & BLO-39-11-14-004 & R610561 & PGMisc-NoU-1-Act \% & $66-01-01-439$ \\
\hline & PGMisc-NoU-2b-NoBe-Act \% & MT1490 & & PGMisc-NoU-2a-Be & ARF-102-85-114-1 \\
\hline & PGMisc-NoU-WG & PPSL-365 & & PGMisc-NoU-WG & PPSL-365 \\
\hline R601385 & PGMisc-NoU-1-Act $\%$ & BLO-39-11-14-004 & R610569 & PGMisc+U-1-U\% & CAN92 \\
\hline & PGMisc-NoU-2b-NoBe-Act $\%$ & MT1490 & & PGMisc+U-2-Act\% & PSU-84-06-05 \\
\hline & PGMisc-NoU-WG & PPSL-365 & & PGMisc+U-3-Be-WG & ARF-102-85-114-1 \\
\hline R610056 & PGMisc-NoU-1-Act $\%$ & $66-01-01-439$ & R610583 & PGMisc+U-1-U\% & 5501407 \\
\hline & PGMisc-NoU-2a-Be & ARF-102-85-114-1 & & PGMisc+U-2-Act\% & PSU-84-06-05 \\
\hline & PGMisc-NoU-WG & PPSL-365 & & PGMisc+U-3-Be-WG & CAN92 \\
\hline R610207 & PGMisC+U-1-U\% & PSU-84-06-05 & R610597 & PGMisc+U-1-U\% & 5501407 \\
\hline & PGMisc+U-2-Act\% & 669194 & & PGMisc+U-2-Act\% & PSU-84-06-05 \\
\hline & PGMisc+U-3-WG & PPSL-365 & & PGMisc+U-3-Be-WG & CAN92 \\
\hline
\end{tabular}




\begin{tabular}{|c|c|c|}
\hline $\begin{array}{c}3013 \\
\text { Container }\end{array}$ & $\begin{array}{l}\text { MIS Represented Item } \\
\text { Selection Order }\end{array}$ & $\begin{array}{c}\text { MIS Represented } \\
\text { Item }\end{array}$ \\
\hline \multirow[t]{3}{*}{ R610601 } & PGMisc+U-1-U\% & 5501407 \\
\hline & PGMisc+U-2-Act\% & PSU-84-06-05 \\
\hline & PGMisc+U-3-Be-WG & \begin{tabular}{|l} 
CAN92 \\
\end{tabular} \\
\hline \multirow[t]{3}{*}{ R610609 } & PGMisc+U-1-U\% & 5501407 \\
\hline & PGMisc+U-2-Act\% & PSU-84-06-05 \\
\hline & PGMisc+U-3-Be-WG & CAN92 \\
\hline \multirow[t]{3}{*}{ R610614 } & PGMisc-NoU-1-Act\% & PPSL-365 \\
\hline & PGMisc-NoU-2b-NoBe-Act\% & BLO-39-11-14-004 \\
\hline & PGMisc-NoU-WG & MT1490 \\
\hline \multirow[t]{3}{*}{ R610619 } & PGMisc-NoU-1-Act $\%$ & $66-01-01-439$ \\
\hline & PGMisc-NoU-2a-Be & ARF-102-85-114-1 \\
\hline & PGMisc-NoU-WG & PPSL-365 \\
\hline \multirow[t]{3}{*}{ R610620 } & PGMisc+U-1-U\% & 5501407 \\
\hline & PGMisc+U-2-Act $\%$ & PSU-84-06-05 \\
\hline & PGMisc+U-3-Be-WG & CAN92 \\
\hline \multirow[t]{3}{*}{ R610621 } & PGMisc+U-1-U\% & 5501407 \\
\hline & PGMisc+U-2-Act\% & PSU-84-06-05 \\
\hline & PGMisc+U-3-Be-WG & CAN92 \\
\hline \multirow[t]{3}{*}{ R610636 } & PGMisc-NoU-1-Act $\%$ & PPSL-365 \\
\hline & PGMisc-NoU-2a-Be & ARF-102-85-114-1 \\
\hline & PGMisc-NoU-WG & MT1490 \\
\hline \multirow[t]{3}{*}{ R610649 } & PGMisc+U-1-U\% & 5501407 \\
\hline & PGMisc+U-2-Act\% & PSU-84-06-05 \\
\hline & PGMisc+U-3-Be-WG & CAN92 \\
\hline \multirow[t]{3}{*}{ R610665 } & PGMisc-NoU-1-Act\% & PPSL-365 \\
\hline & PGMisc-NoU-2a-Be & ARF-102-85-114-1 \\
\hline & PGMisc-NoU-WG & $66-01-01-439$ \\
\hline \multirow[t]{3}{*}{ R610675 } & PGMisc-NoU-1-Act \% & $66-01-01-439$ \\
\hline & PGMisc-NoU-2a-Be & ARF-102-85-114-1 \\
\hline & PGMisc-NoU-WG & PPSL-365 \\
\hline \multirow[t]{3}{*}{ R610690 } & PGMisc-NoU-1-Act\% & $66-01-01-439$ \\
\hline & PGMisc-NoU-2a-Be & ARF-102-85-114-1 \\
\hline & PGMisc-NoU-WG & PPSL-365 \\
\hline \multirow[t]{3}{*}{ R610738 } & PGMisc-NoU-1-Act\% & $66-01-01-439$ \\
\hline & PGMisc-NoU-2a-Be & ARF-102-85-114-1 \\
\hline & PGMisc-NoU-WG & PPSL-365 \\
\hline \multirow[t]{3}{*}{ R610752 } & PGMisc-NoU-1-Act\% & PPSL-365 \\
\hline & PGMisc-NoU-2a-Be & ARF-102-85-114-1 \\
\hline & PGMisc-NoU-WG & MT1490 \\
\hline \multirow[t]{3}{*}{ R610809 } & PGMisc-NoU-1-Act $\%$ & $66-01-01-439$ \\
\hline & PGMisc-NoU-2b-NoBe-Act\% & PPSL-365 \\
\hline & PGMisc-NoU-WG & MT1490 \\
\hline \multirow[t]{3}{*}{ R610843 } & PGMisc-NoU-1-Act $\%$ & PPSL-365 \\
\hline & PGMisc-NoU-2a-Be & ARF-102-85-114-1 \\
\hline & PGMisc-NoU-WG & MT1490 \\
\hline \multirow[t]{3}{*}{ R610846 } & PGMisc+U-1-U\% & CAN92 \\
\hline & PGMisc+U-2-Act $\%$ & 5501407 \\
\hline & PGMisc+U-3-Be-WG & ARF-102-85-114-1 \\
\hline \multirow[t]{3}{*}{$R 610847$} & PGMisc-NoU-1-Act \% & PBO-47-09-012-023 \\
\hline & PGMisc-NoU-2a-Be & ARF-102-85-114-1 \\
\hline & PGMisc-NoU-WG & MT1490 \\
\hline \multirow[t]{3}{*}{ R610848 } & PGMisc-NoU-1-Act\% & $66-01-01-439$ \\
\hline & PGMisc-NoU-2a-Be & ARF-102-85-114-1 \\
\hline & PGMisc-NoU-WG & PPSL-365 \\
\hline \multirow[t]{3}{*}{ R610870 } & PGMisc-NoU-1-Act\% & PPSL-365 \\
\hline & PGMisc-NoU-2a-Be & ARF-102-85-114-1 \\
\hline & PGMisc-NoU-WG & MT1490 \\
\hline
\end{tabular}

\begin{tabular}{|c|c|c|}
\hline $\begin{array}{c}3013 \\
\text { Container } \\
\end{array}$ & $\begin{array}{l}\text { MIS Represented Item } \\
\text { Selection Order }\end{array}$ & $\begin{array}{l}\text { MIS Represented } \\
\text { Item }\end{array}$ \\
\hline \multirow[t]{3}{*}{ R610874 } & PGMisc+U-1-U\% & CAN92 \\
\hline & PGMisc+U-2-Act $\%$ & 5501407 \\
\hline & PGMisc+U-3-Be-WG & ARF-102-85-114-1 \\
\hline \multirow[t]{3}{*}{ R610876 } & PGMisc-NoU-1-Act\% & PPSL-365 \\
\hline & PGMisc-NoU-2a-Be & ARF-102-85-114-1 \\
\hline & PGMisc-NoU-WG & MT1490 \\
\hline \multirow[t]{3}{*}{ R610878 } & PGMisc-NoU-1-Act\% & $66-01-01-439$ \\
\hline & PGMisc-NoU-2a-Be & ARF-102-85-114-1 \\
\hline & PGMisc-NoU-WG & PPSL-365 \\
\hline \multirow[t]{3}{*}{ R610879 } & PGMisc-NoU-1-Act\% & $66-01-01-439$ \\
\hline & PGMisc-NoU-2a-Be & ARF-102-85-114-1 \\
\hline & PGMisc-NoU-NWG & PBO-47-09-012-023 \\
\hline \multirow[t]{3}{*}{ R610888 } & PGMisc-NoU-1-Act\% & PBO-47-09-012-023 \\
\hline & PGMisc-NoU-2a-Be & ARF-102-85-114-1 \\
\hline & PGMisc-NoU-WG & MT1490 \\
\hline \multirow[t]{3}{*}{ R610890 } & PGMisc-NoU-1-Act\% & $66-01-01-439$ \\
\hline & PGMisc-NoU-2a-Be & ARF-102-85-114-1 \\
\hline & PGMisc-NoU-WG & PPSL-365 \\
\hline \multirow[t]{3}{*}{ R610897 } & PGMisc-NoU-1-Act\% & PPSL-365 \\
\hline & PGMisc-NoU-2a-Be & ARF-102-85-114-1 \\
\hline & PGMisc-NoU-WG & $66-01-01-439$ \\
\hline \multirow[t]{3}{*}{ R610907 } & PGMisc-NoU-1-Act\% & PPSL-365 \\
\hline & PGMisc-NoU-2a-Be & ARF-102-85-114-1 \\
\hline & PGMisc-NoU-WG & MT1490 \\
\hline \multirow[t]{3}{*}{ R610922 } & PGMisc-NoU-1-Act\% & PPSL-365 \\
\hline & PGMisc-NoU-2a-Be & ARF-102-85-114-1 \\
\hline & PGMisc-NoU-WG & MT1490 \\
\hline \multirow[t]{3}{*}{ R610923 } & PGMisc+U-1-U\% & CAN92 \\
\hline & PGMisc+U-2-Act\% & 5501407 \\
\hline & PGMisc+U-3-Be-WG & ARF-102-85-114-1 \\
\hline \multirow[t]{3}{*}{ R610928 } & PGMisc-NoU-1-Act $\%$ & PPSL-365 \\
\hline & PGMisc-NoU-2a-Be & ARF-102-85-114-1 \\
\hline & PGMisc-NoU-WG & MT1490 \\
\hline \multirow[t]{3}{*}{ R610929 } & PGMisc+U-1-U\% & CAN92 \\
\hline & PGMisc+U-2-Act $\%$ & 5501407 \\
\hline & PGMisc+U-3-Be-WG & ARF-102-85-114-1 \\
\hline \multirow[t]{3}{*}{ R610951 } & PGMisc+U-1-U\% & CAN92 \\
\hline & PGMisc+U-2-Act\% & 669194 \\
\hline & PGMisc+U-3-Be-WG & ARF-102-85-114-1 \\
\hline \multirow[t]{3}{*}{ R610952 } & PGMisc+U-1-U\% & CAN92 \\
\hline & PGMisc+U-2-Act\% & 5501407 \\
\hline & PGMisc+U-3-Be-WG & ARF-102-85-114-1 \\
\hline \multirow[t]{3}{*}{ R610984 } & PGMisc-NoU-1-Act $\%$ & $66-01-01-439$ \\
\hline & PGMisc-NoU-2a-Be & ARF-102-85-114-1 \\
\hline & PGMisc-NoU-WG & PPSL-365 \\
\hline \multirow[t]{3}{*}{ R611007 } & PGMisc-NoU-1-Act $\%$ & $66-01-01-439$ \\
\hline & PGMisc-NoU-2a-Be & ARF-102-85-114-1 \\
\hline & PGMisc-NoU-WG & PPSL-365 \\
\hline \multirow[t]{3}{*}{ R611042 } & PGMisc-NoU-1-Act\% & $66-01-01-439$ \\
\hline & PGMisc-NoU-2a-Be & ARF-102-85-114-1 \\
\hline & PGMisc-NoU-WG & PPSL-365 \\
\hline \multirow[t]{3}{*}{$R 611080$} & PGMisc-NoU-1-Act\% & $66-01-01-439$ \\
\hline & PGMisc-NoU-2a-Be & ARF-102-85-114-1 \\
\hline & PGMisc-NoU-WG & PPSL-365 \\
\hline \multirow[t]{3}{*}{ R611103 } & PGMisc-NoU-1-Act $\%$ & $66-01-01-439$ \\
\hline & PGMisc-NoU-2a-Be & ARF-102-85-114-1 \\
\hline & PGMisc-NoU-WG & PPSL-365 \\
\hline
\end{tabular}




\begin{tabular}{|c|c|c|}
\hline $\begin{array}{c}3013 \\
\text { Container }\end{array}$ & $\begin{array}{c}\text { MIS Represented Item } \\
\text { Selection Order }\end{array}$ & $\begin{array}{c}\text { MIS Represented } \\
\text { Item }\end{array}$ \\
\hline \multirow[t]{3}{*}{ R611284 } & PGMisc+U-1-U\% & CAN92 \\
\hline & PGMisc+U-2-Act $\%$ & 5501407 \\
\hline & PGMisc+U-3-Be-WG & ARF-102-85-114-1 \\
\hline \multirow[t]{3}{*}{ R611305 } & PGMisc+U-1-U\% & PSU-84-06-05 \\
\hline & PGMisc+U-2-Act\% & 5501407 \\
\hline & PGMisc+U-3-WG & 669194 \\
\hline \multirow[t]{3}{*}{ R611320 } & PGMisc-NoU-1-Act\% & PPSL-365 \\
\hline & PGMisc-NoU-2a-Be & ARF-102-85-114-1 \\
\hline & PGMisc-NoU-WG & MT1490 \\
\hline \multirow[t]{3}{*}{ R611379 } & PGMisc+U-1-U\% & 5501407 \\
\hline & PGMisc+U-2-Act\% & CAN92 \\
\hline & PGMisc+U-3-WG & MT1490 \\
\hline \multirow[t]{3}{*}{ R611419 } & PGMisc+U-1-U\% & CAN92 \\
\hline & PGMisc+U-2-Act\% & 5501407 \\
\hline & PGMisc+U-3-Be-WG & ARF-102-85-114-1 \\
\hline \multirow[t]{3}{*}{$\overline{R 611424}$} & PGMisc-NoU-1-Act\% & $66-01-01-439$ \\
\hline & PGMisc-NoU-2a-Be & ARF-102-85-114-1 \\
\hline & PGMisc-NoU-NWG & PBO-47-09-012-023 \\
\hline \multirow[t]{3}{*}{$\mathrm{H} 002453$} & PGMisc+U-1-U\% & PSU-84-06-05 \\
\hline & PGMisC+U-2-Act\% & 5501407 \\
\hline & PGMisc+U-3-NWG & PBO-47-09-012-023 \\
\hline \multirow[t]{3}{*}{5002173} & PGMisc+U-1-U\% & 669194 \\
\hline & PGMisC+U-2-ACt\% & CAN92 \\
\hline & PGMisc+U-3-NWG & PSU-84-06-05 \\
\hline \multirow[t]{3}{*}{ H000771 } & PGNolmp & 5501579 \\
\hline & & PEOF1 \\
\hline & & SCP711-56 \\
\hline \multirow[t]{3}{*}{ H000799 } & PGNolmp & 5501579 \\
\hline & & PEOF1 \\
\hline & & SCP711-56 \\
\hline \multirow[t]{3}{*}{ H000807 } & PGNolmp & 5501579 \\
\hline & & PEOF1 \\
\hline & & SCP711-56 \\
\hline \multirow[t]{3}{*}{ H000841 } & PGNolmp & 5501579 \\
\hline & & \begin{tabular}{|l|} 
PEOF1 \\
\end{tabular} \\
\hline & & SCP711-56 \\
\hline \multirow[t]{3}{*}{ H000904 } & PGNolmp & 5501579 \\
\hline & & PEOF1 \\
\hline & & SCP711-56 \\
\hline \multirow[t]{3}{*}{ H000922 } & PGNolmp & 5501579 \\
\hline & & PEOF1 \\
\hline & & SCP711-56 \\
\hline \multirow[t]{3}{*}{ H002293 } & PGNolmp & 5501579 \\
\hline & & PEOF1 \\
\hline & & SCP711-56 \\
\hline \multirow[t]{3}{*}{ H002485 } & PGNolmp & 5501579 \\
\hline & & PEOF1 \\
\hline & & SCP711-56 \\
\hline \multirow[t]{3}{*}{ H002655 } & PGNolmp & 5501579 \\
\hline & & PEOF1 \\
\hline & & SCP711-56 \\
\hline \multirow[t]{3}{*}{ H002714 } & PGNolmp & 5501579 \\
\hline & & PEOF1 \\
\hline & & SCP711-56 \\
\hline \multirow[t]{3}{*}{$\mathrm{H} 002730$} & PGNolmp & 5501579 \\
\hline & & PEOF1 \\
\hline & & SCP711-56 \\
\hline
\end{tabular}

\begin{tabular}{|c|c|c|}
\hline $\begin{array}{c}3013 \\
\text { Container }\end{array}$ & $\begin{array}{l}\text { MIS Represented Item } \\
\text { Selection Order }\end{array}$ & $\begin{array}{c}\text { MIS Represented } \\
\text { Item }\end{array}$ \\
\hline \multirow[t]{3}{*}{ L000062 } & PGNolmp & 5501579 \\
\hline & & PEOF1 \\
\hline & & SCP711-56 \\
\hline \multirow[t]{3}{*}{ L000063 } & PGNolmp & 5501579 \\
\hline & & PEOF1 \\
\hline & & SCP711-56 \\
\hline \multirow[t]{3}{*}{ L000154 } & PGNolmp & 5501579 \\
\hline & & PEOF1 \\
\hline & & SCP711-56 \\
\hline \multirow[t]{3}{*}{ L000193 } & PGNolmp & 5501579 \\
\hline & & PEOF1 \\
\hline & & SCP711-56 \\
\hline \multirow[t]{3}{*}{$\mathrm{H} 001960$} & PGNolmp & 5501579 \\
\hline & & PEOF1 \\
\hline & & SCP711-56 \\
\hline \multirow[t]{3}{*}{$\mathrm{H} 002227$} & PGNolmp & 5501579 \\
\hline & & PEOF1 \\
\hline & & SCP711-56 \\
\hline \multirow[t]{3}{*}{$\mathrm{H} 002360$} & PGNolmp & 5501579 \\
\hline & & PEOF1 \\
\hline & & SCP711-56 \\
\hline \multirow[t]{3}{*}{ S001736 } & PGNoImp & 5501579 \\
\hline & & PEOF1 \\
\hline & & SCP711-56 \\
\hline \multirow[t]{3}{*}{$\$ 002204$} & PGNoImp & 5501579 \\
\hline & & PEOF1 \\
\hline & & SCP711-56 \\
\hline \multirow[t]{3}{*}{ S002237 } & PGNolmp & 5501579 \\
\hline & & \begin{tabular}{|l|} 
PEOF1 \\
\end{tabular} \\
\hline & & SCP711-56 \\
\hline \multirow[t]{3}{*}{ R610887 } & PGMisc-NoU-1-Act\% & PPSL-365 \\
\hline & PGMisc-NoU-2a-Be & ARF-102-85-114-1 \\
\hline & PGMisc-NoU-WG & MT1490 \\
\hline
\end{tabular}




\section{Appendix E: 3013 Container Outliers}


The 3013 containers listed below are identified as outliers because chlorine was detected by PG analysis. These materials are not expected to have chlorine as an impurity, based on process history.

\begin{tabular}{|c|c|c|c|c|c|c|c|c|c|c|c|}
\hline 3013 Taxon & $\begin{array}{c}\text { Old MIS } \\
\text { Represented } \\
\text { Code }\end{array}$ & $\begin{array}{c}\text { Material } \\
\text { Description }\end{array}$ & $\begin{array}{c}3013 \\
\text { Container ID }\end{array}$ & PG Result & $\begin{array}{c}\mathrm{Pu} \\
\mathrm{wt} \%\end{array}$ & $\underset{\mathbf{w t} \%}{U}$ & $\begin{array}{l}\text { Actinide } \\
\text { wt\% }\end{array}$ & $\begin{array}{c}\mathrm{Cl} \\
\text { wt\% } \\
\text { (est.) }\end{array}$ & $\begin{array}{l}\mathrm{H}_{2} \mathrm{O} \\
\text { wt\% }\end{array}$ & $\begin{array}{l}\text { Moisture } \\
\text { Method }\end{array}$ & MIS Represented Items \\
\hline \multirow{9}{*}{ OxIPPt-HN } & \multirow{9}{*}{ Hanford-1B } & \multirow{9}{*}{$\begin{array}{l}\text { Product quality } \\
\text { oxides from oxalate } \\
\text { precipitation - } \\
\text { (stored product) }\end{array}$} & H002543 & Chloride & 86.0 & 0.0 & 86.8 & 0.9 & 0.19 & TGA & $\begin{array}{l}\text { 053038, TS707001, } \\
\text { ARF-102-85-365 }\end{array}$ \\
\hline & & & $\mathrm{H} 002777$ & Chloride & 84.2 & 0.0 & 84.8 & 1.5 & 0.04 & TGA & $\begin{array}{c}\text { 053038, TS707001, } \\
\text { ARF-102-85-365 }\end{array}$ \\
\hline & & & H002863 & Chloride & 82.6 & 0.0 & 86.1 & 6.7 & 0.07 & TGA & $\begin{array}{l}\text { CLLANL025, C00695, } \\
\text { ARF-102-85-295 }\end{array}$ \\
\hline & & & H002864 & Chloride & 86.3 & 0.0 & 87.4 & 1.4 & 0.01 & TGA & $\begin{array}{c}\text { 053038, TS707001, } \\
\text { ARF-102-85-365 }\end{array}$ \\
\hline & & & H003014 & $\begin{array}{c}\text { Chloride } \\
\text { Containing Be \& F }\end{array}$ & 82.3 & 0.0 & 85.6 & 1.6 & 0.05 & TGA & $\begin{array}{c}\text { 053038, TS707001, } \\
\text { ARF-102-85-365 }\end{array}$ \\
\hline & & & $\mathrm{H} 004033$ & $\begin{array}{c}\text { Chloride } \\
\text { Containing } \mathrm{F} \\
\end{array}$ & 86.0 & 0.0 & 87.1 & 3.9 & 0.02 & TGA & $\begin{array}{l}\text { ARF-102-85-223, PMAXBS, } \\
\text { CLLANL025 }\end{array}$ \\
\hline & & & $\mathrm{H} 004150$ & $\begin{array}{c}\text { Chloride } \\
\text { Containing Be \& F }\end{array}$ & 86.5 & 0.0 & 87.3 & 2.4 & 0.02 & TGA & $\begin{array}{c}\text { 053038, TS707001, } \\
\text { ARF-102-85-365 }\end{array}$ \\
\hline & & & $\mathrm{H} 004206$ & $\begin{array}{c}\text { Chloride } \\
\text { Containing F } \\
\end{array}$ & 78.0 & 0.0 & 85.8 & 1.8 & 0.04 & TGA & $\begin{array}{c}\text { 053038, TS707001, } \\
\text { ARF-102-85-365 } \\
\end{array}$ \\
\hline & & & H004309 & $\begin{array}{c}\text { Chloride } \\
\text { Containing Be \& F }\end{array}$ & 82.7 & 0.0 & 86.7 & 5.4 & 0.11 & TGA & $\begin{array}{c}\text { ARF-102-85-223, PMAXBS, } \\
\text { CLLANL025 }\end{array}$ \\
\hline \multirow{3}{*}{$\begin{array}{l}\text { PuUOx-HN- } \\
\text { Pure }\end{array}$} & \multirow{3}{*}{ Hanford-2D } & \multirow{3}{*}{$\begin{array}{l}\text { Mixed oxides: } \\
\text { product quality and } \\
\text { impure }\end{array}$} & H002195 & Chloride & 21.0 & 62.3 & 84.2 & 1.5 & 0.11 & TGA & $\begin{array}{c}\text { 053038, TS707001, } \\
\text { ARF-102-85-365 } \\
\end{array}$ \\
\hline & & & H002812 & $\begin{array}{c}\text { Chloride } \\
\text { Containing F }\end{array}$ & 20.7 & 64.8 & 85.8 & 8.4 & 0.14 & TGA & $\begin{array}{l}\text { CLLANL025, C00695, } \\
\text { ARF-102-85-295 }\end{array}$ \\
\hline & & & H003057 & Chloride & 24.9 & 56.3 & 81.4 & 0.2 & 0.13 & TGA & $\begin{array}{c}\text { 07032282A, } \\
\text { ARF-102-85-365, C00024A }\end{array}$ \\
\hline \multirow{2}{*}{ ScrapOx-HN-Hi } & \multirow{2}{*}{ Hanford-2A } & \multirow{2}{*}{$\begin{array}{l}\text { Impure and scrap } \\
\text { Pu oxides with } 80- \\
85 \text { wt } \% \text { Pu from } \\
\text { PFP and } 300 \text { area }\end{array}$} & H002673 & Chloride & 81.5 & 0.0 & 81.7 & 1.3 & 0.06 & TGA & $\begin{array}{c}\text { 053038, TS707001, } \\
\text { ARF-102-85-365 }\end{array}$ \\
\hline & & & H002886 & $\begin{array}{c}\text { Chloride } \\
\text { Containing F }\end{array}$ & 82.8 & 0.0 & 83.6 & 3.0 & 0.09 & TGA & $\begin{array}{l}\text { 053038, TS707001, } \\
\text { ARF-102-85-365 }\end{array}$ \\
\hline HydrOx-RF & RFETS-2D & $\begin{array}{c}\text { By-product hydride } \\
\text { oxidation }\end{array}$ & H000857 & Chloride & 7.6 & 66.2 & 74.0 & 1.6 & 0.01 & FTIR & $\begin{array}{c}\text { 053038, TS707001, } \\
\text { ARF-102-85-365 }\end{array}$ \\
\hline PeroxPPT-RF & RFETS-1A & $\begin{array}{l}\text { Peroxide } \\
\text { precipitation/ } \\
\text { calcination }\end{array}$ & R610836 & $\begin{array}{l}\text { Chloride } \\
\text { Containing F }\end{array}$ & 83.7 & 0.0 & 83.9 & 0.9 & 0.03 & TGA & $\begin{array}{l}\text { 053038, TS707001, } \\
\text { ARF-102-85-365 }\end{array}$ \\
\hline MetalOx-SR-LL & SRS-1D & $\begin{array}{c}\text { Burned metal } \\
\text { oxides from LLNL } \\
>80 \%\end{array}$ & S001721 & $\begin{array}{c}\text { Chloride } \\
\text { Containing F }\end{array}$ & 84.3 & 0.0 & 84.3 & 2.0 & 0.21 & TGA & $\begin{array}{l}\text { 053038, TS707001, } \\
\text { ARF-102-85-365 }\end{array}$ \\
\hline $\begin{array}{l}\text { PuUOx-SR- } \\
\text { Misc }\end{array}$ & SRS-2B & $\begin{array}{l}\text { Scrap mixed Pu/U } \\
\text { oxides (Other } \\
\text { sources) }\end{array}$ & S002147 & $\begin{array}{c}\text { Chloride } \\
\text { Containing F }\end{array}$ & 74.4 & 0.0 & 74.4 & 0.7 & 0.20 & TGA & $\begin{array}{l}\text { 053038, TS707001, } \\
\text { ARF-102-85-365 }\end{array}$ \\
\hline
\end{tabular}


The 3013 containers listed below are identified as outliers because fluorine was detected by PG analysis in concentrations greater that $0.8 \mathrm{wt} \%$. These materials are not expected to have fluorine as an impurity at these concentrations, based on process history. Based on the PG representation, the containers in this table would be represented by 07242201A, 64-85-12-1858, and PuF4-1.

\begin{tabular}{|c|c|c|c|c|c|c|c|c|c|c|}
\hline 3013 Taxon & $\begin{array}{c}\text { Old MIS } \\
\text { Represented } \\
\text { Code }\end{array}$ & Material Description & $\begin{array}{c}3013 \\
\text { Container ID }\end{array}$ & PG Result & $\begin{array}{c}\mathrm{Pu} \\
\mathrm{wt} \%\end{array}$ & $\underset{\mathbf{w t} \%}{U}$ & $\begin{array}{c}\text { Actinide } \\
\text { wt } \%\end{array}$ & $\begin{array}{l}\text { F wt\% } \\
\text { (est.) }\end{array}$ & $\begin{array}{l}\mathrm{H}_{2} \mathrm{O} \\
\mathrm{wt} \%\end{array}$ & $\begin{array}{l}\text { Moisture } \\
\text { Method }\end{array}$ \\
\hline \multirow{5}{*}{ OxIPPt-HN } & \multirow{5}{*}{ Hanford-1B } & \multirow{5}{*}{$\begin{array}{l}\text { Product quality oxides } \\
\text { from oxalate } \\
\text { precipitation - (Stored } \\
\text { product) }\end{array}$} & $\mathrm{H} 002801$ & Strong Fluoride & 84.8 & 0.0 & 87.1 & 2.7 & 0.02 & TGA \\
\hline & & & $\mathrm{H} 003153$ & Strong Fluoride & 85.1 & 0.0 & 87.4 & 2.4 & 0.03 & TGA \\
\hline & & & H003157 & Strong Fluoride & 81.1 & 0.0 & 85.0 & 1.1 & 0.19 & TGA \\
\hline & & & H004009 & Strong Fluoride & 80.4 & 0.0 & 80.6 & 1.6 & 0.20 & TGA \\
\hline & & & $\mathrm{H} 004175$ & Strong Fluoride & 86.5 & 0.0 & 87.3 & 1.8 & 0.02 & TGA \\
\hline \multirow{2}{*}{ PuUOx-HN-Pure } & \multirow{2}{*}{ Hanford-2D } & \multirow{2}{*}{$\begin{array}{l}\text { Mixed oxides: product } \\
\text { quality and impure }\end{array}$} & $\mathrm{H} 002862$ & Strong Fluoride & 19.2 & 66.1 & 85.6 & 4.6 & 0.14 & TGA \\
\hline & & & $\mathrm{H} 003107$ & Strong Fluoride & 69.3 & 17.3 & 87.6 & 3.5 & 0.14 & TGA \\
\hline ScrapOx-HN-Hi & Hanford-2A & $\begin{array}{c}\text { Impure and scrap Pu } \\
\text { oxides with } 80-85 \text { wt\% } \\
\text { Pu from PFP and } 300 \\
\text { area } \\
\end{array}$ & H003314 & Strong Fluoride & 80.1 & 0.0 & 80.9 & 3.0 & 0.09 & TGA \\
\hline \multirow{7}{*}{ PuUOx-SR-Misc } & \multirow{7}{*}{ SRS-2B } & \multirow{7}{*}{$\begin{array}{c}\text { Scrap mixed oxides - a) } \\
\text { AUA-265 (CaF, LANL, } \\
\text { HRA), b) ORNL, c) Misc. } \\
\text { Sources most }>80 \% \\
\text { Pu + U, d) SRTC returns }\end{array}$} & S002105 & Strong Fluoride & 81.3 & 0.0 & 81.3 & 1.0 & 0.14 & TGA \\
\hline & & & S002110 & Strong Fluoride & 57.1 & 0.0 & 57.1 & 1.9 & 0.39 & TGA \\
\hline & & & S002220 & Strong Fluoride & 12.9 & 74.4 & 87.3 & 11.7 & 0.38 & TGA \\
\hline & & & S002222 & Strong Fluoride & 40.3 & 24.9 & 65.2 & 1.6 & 0.18 & TGA \\
\hline & & & S002238 & Strong Fluoride & 76.0 & 1.4 & 77.5 & 2.2 & 0.18 & TGA \\
\hline & & & S002250 & Strong Fluoride & 60.6 & 0.0 & 60.6 & 6.0 & 0.16 & TGA \\
\hline & & & S002276 & Strong Fluoride & 37.3 & 27.1 & 64.5 & 1.8 & 0.33 & TGA \\
\hline
\end{tabular}


This report has been reproduced directly from the best available copy. It is available electronically on the Web (http://www.doe.gov/bridge).

Copies are available for sale to U.S. Department of Energy employees and contractors from:

Office of Scientific and Technical Information

P.O. Box 62

Oak Ridge, TN 37831

(865) 576-8401

Copies are available for sale to the public from: National Technical Information Service

U.S. Department of Commerce

5285 Port Royal Road

Springfield, VA 22161

(800) 553-6847 
- Los Alamos NATIONAL LABORATORY 